\title{
ON EQUIVALENCE OF LINEAR CONTROL SYSTEMS AND ITS USAGE TO VERIFICATION OF THE ADEQUACY OF DIFFERENT MODELS FOR A REAL DYNAMIC PROCESS
}

\author{
Vasiliy Ye. Belozyorov*, Danylo V. Dantsev ${ }^{\dagger}$ Svetlana A. Volkova ${ }^{\ddagger}$
}

\begin{abstract}
A problem of description of algebraic invariants for a linear control system that determine its structure is considered. With the help of these invariants, the equivalence problem of two linear time-invariant control systems with respect to actions of some linear groups on the spaces of inputs, outputs, and states of these systems is solved. The invariants are used to establish the necessary equivalence conditions for two nonlinear systems of differential equations generalizing the well-known Hopfield neural network model. Finally, these conditions are applied to establish the adequacy of two neural network models designed to describe the behavior of a real dynamic process given by two different sets of time series.
\end{abstract}

Key words: linear time-invariant control system, system of ordinary autonomous differential equations, complete linear group, special linear group, exterior degree, algebraic invariant, null-form, neural network, activation function.

2010 Mathematics Subject Classification: 13A50, 14L24,15A72, 93C05.

Communicated by Prof. V. Ye. Kapustyan

\section{Introduction}

In recent decades, researchers have paid much attention to chaotic behavior in many fields, such as meteorology, medicine, economics, signal processing, traffic flow, and many others $[16,34,36,49]$. They also developed many models describing chaotic time series in order to predict the behavior of these time series. Researchers have found that it is a difficult problem to forecast chaotic time series, which are the evolution of chaotic systems, with the use of traditional time series forecasting methods $[16,36]$. Now chaos theory has become an important part of nonlinear science and is used for forecasting chaotic time series. Therefore, modeling of chaotic systems constructed from observed data and predicting multiple future values of the time series has become an important issue [16, 34, 36, 49].

We will assume that we know the dimension $n$ of the real phase space in which the considered dynamic process $\mathbf{P}(t) \in \mathbb{R}^{n}$ takes place [7]. Further, for modeling of the process $\mathbf{P}(t)=\left(P_{1}(t), \ldots, P_{n}(t)\right)^{T}$ neural networks will be used $[17,25,26]$. The motivation for this use is given below.

In the beginning we give a generalization of one well-known result of approximation theory of functions:

\footnotetext{
* Department of Applied Mathematics, Oles Honchar Dnipro National University, 72, Gagarin's Avenue, 49010, Dnipro, Ukraine, belozvye2017@gmail.com

${ }^{\dagger}$ Chaotic Dynamics Inc., 201-45793, Luckakuck Way, Chilliwack BC V2R 5S3, Canada, danylo@chaodyna.com

${ }^{\ddagger}$ Department of Computing Engineering and Applied Mathematics, Ukrainian State University of Chemical Technology, 8, Gagarin's Avenue, 49005, Dnipro, Ukraine, svolkova2017@gmail.com

(C) V. Ye. Belozyorov, D. V. Dantsev, S. A. Volkova, 2020.
} 
Theorem 1.1. [17] Let $\phi(z): \mathbb{R} \rightarrow \mathbb{R}$ be a nonconstant, bounded, and monotoneincreasing continuous nonlinear function. Let also $f\left(x_{1}, \ldots, x_{n}\right): \mathbb{R}^{n} \rightarrow \mathbb{R}$ be any given continuous function. Then, $\forall \epsilon>0$ there exist an integer $k>0$ and sets of real constants $\alpha_{i}, \beta_{i}$, and $\gamma_{i j}$, where $i=1, \ldots, k ; j=1, \ldots, n$ such that we may define the function

$$
F\left(x_{1}, \ldots, x_{n}\right)=\sum_{i=1}^{k} \alpha_{i} \phi\left(\sum_{j=1}^{n} \gamma_{i j} x_{j}+\beta_{i}\right)
$$

as an approximation realization of the function $f\left(x_{1}, \ldots, x_{n}\right)$ :

$$
\forall x_{1}, \ldots, x_{n} \in \mathbb{R}\left|F\left(x_{1}, \ldots, x_{n}\right)-f\left(x_{1}, \ldots, x_{n}\right)\right|<\epsilon .
$$

The function $\phi(z): \mathbb{R} \rightarrow \mathbb{R}$ is called an activation function $[17,26]$. (In most scientific publications is suggested that $\phi(z): \mathbb{R} \rightarrow[0,1]$ is a sigmoid function. However, in some cases, the condition that the activation function $\phi(x)$ is bounded can be removed [48].)

In the future, we will use the version of Theorem 1.1, in which one activation function $\phi(z)$ will be replaced by several similar functions $\phi_{1}(z), \ldots, \phi_{k}(z)$ :

$$
F\left(x_{1}, \ldots, x_{n}\right)=\sum_{i=1}^{k} \alpha_{i} \phi_{i}\left(\sum_{j=1}^{n} \gamma_{i j} x_{j}+\beta_{i}\right) .
$$

Let

$$
x_{0}=x\left(t_{0}\right), x_{1}=x\left(t_{1}\right), \ldots, x_{N}=x\left(t_{N}\right)
$$

be a finite sequence of numerical values of some scalar dynamical variable $x(t)$ measured with the constant time step $\Delta t$ in the moments $t_{i}=t_{0}+i \Delta t ; x_{i}=x\left(t_{i}\right)$; $i=0,1, \ldots, N$. Sequence (1.1) is called a time series [29] - [31], [34], [49].

Suppose that we know $n$ time series

$$
\left\{\begin{array}{c}
P_{10}, P_{11}, P_{12}, \ldots, P_{1 N} \\
P_{20}, P_{21}, P_{22}, \ldots, P_{2 N} \\
\ldots \ldots \ldots \ldots \ldots . \\
P_{n 0}, P_{n 1}, P_{n 2}, \ldots, P_{n N}
\end{array}\right.
$$

that describe the components of the process $\mathbf{P}(t)=\left(P_{1}(t), \ldots, P_{n}(t)\right)^{T}$ (here $N>>$ $n)$.

Further, we propose an approximate procedure for determining unknown righthand sides of the differential equations. The procedure is based on the leastsquares method and the fact that we know with sufficient precision the values of $\mathbf{x}(t)$ and its derivatives of order equal to the equation order. In this case we avoid considering a possible ill-posed problem by applying consecutive smoothing procedures leading to shortening the given time series (see $[16,36]$ ). 
Let $(\mathbf{u}, \mathbf{v})$ be a scalar product of real vectors $\mathbf{u}, \mathbf{v} \in \mathbb{R}^{n}$. Introduce the real matrices $A \in \mathbb{R}^{n \times n}, B \in \mathbb{R}^{n \times m}, C \in \mathbb{R}^{m \times n}$, and real vectors $\mathbf{d}=\left(d_{1}, \ldots, d_{n}\right)^{T}, \mathbf{c}_{j}=$ $\left(c_{j 1}, \ldots, c_{j n}\right) \in \mathbb{R}^{n} ; j=1, \ldots, m \leq n$.

We assume that using suitable methods (based on Theorem 1.1) the system of ordinary autonomous differential equations, the solution $\mathbf{x}(t) \in \mathbb{R}^{n}$ of which simulates process $\mathbf{P}(t)$ with a given accuracy, was reconstructed [7].

We assume that this system (with the known vector of initial values $\mathbf{x}^{T}(0)=$ $\left.\left(x_{10}, \ldots, x_{n 0}\right)\right)$ has the following form:

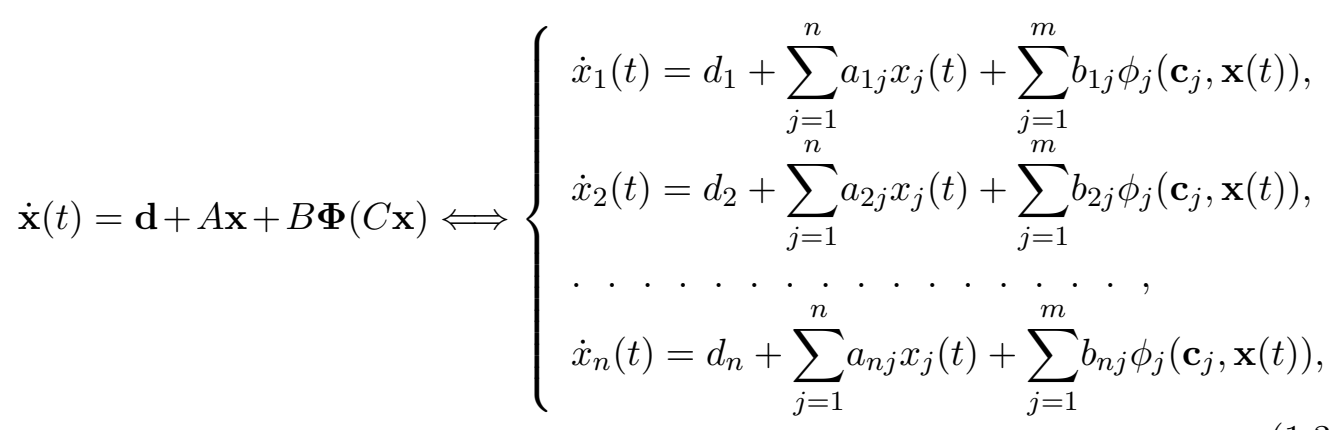

where $\boldsymbol{\Phi}(\mathbf{u})=\left(\phi_{1}\left(u_{1}\right), \ldots, \phi_{m}\left(u_{m}\right)\right)^{T}$. (Note the system (1.3) can be interpreted as a generalized Hopfield neural network (see [25,48]) with activation functions $\left.\phi_{1}\left(u_{1}\right), \ldots, \phi_{m}\left(u_{m}\right).\right)$

The form of system (1.3) is dictated by the following considerations: the nonlinear parts of system (1.3) are continuous functions, and therefore Theorem 1.1 was used to describe them; the presence of a linear part makes it possible to use linearization methods to study the stability of solutions of system (1.3).

There is only one serious flaw in the research plan outlined above. This disadvantage lies in the insufficient verification of the adequacy of the constructed model and the process under study. In this paper, mathematical tools have been developed to test the adequacy, based on an algebraic theory of invariants. The idea of such verification is based on the following well-known fact: with arbitrary observations of a dynamic process, there are always functions that are independent of the methods of observations, but depend on an internal structure that determines the behavior of the process. Functions describing this structure are called invariants.

Currently, the construction of a complete set of invariants describing arbitrary nonlinear dynamical systems is an unsolved problem. Therefore, in this article, the approach based on obtaining the missing invariants for the studied nonlinear system using known invariants obtained for a special linear system, was developed. (Immediately, we note that the construction of such invariants may also have an independent mathematical interest.)

Suppose that the same dynamic process $\mathbf{P}(t) \in \mathbb{R}^{n}$ is given by another set of 
time series

$$
\left\{\begin{array}{c}
Q_{10}, Q_{11}, Q_{12}, \ldots, Q_{1 N} \\
Q_{20}, Q_{21}, Q_{22}, \ldots, Q_{2 N} \\
\ldots \ldots \ldots \ldots \ldots . \\
Q_{n 0}, Q_{n 1}, Q_{n 2}, \ldots, Q_{n N}
\end{array}\right.
$$

Instead of variable $\mathbf{x}$, we introduce a new phase variable $\mathbf{y} \in \mathbb{R}^{n}$. Introduce also the real matrices $F \in \mathbb{R}^{n \times n}, G \in \mathbb{R}^{n \times m}, H \in \mathbb{R}^{m \times n}$, and real vectors $\mathbf{l}=$ $\left(l_{1}, \ldots, l_{n}\right)^{T}, \mathbf{h}_{j}=\left(h_{j 1}, \ldots, h_{j n}\right) \in \mathbb{R}^{n} ; j=1, \ldots, m \leq n$.

We also assume that in this case other activation functions $\psi_{1}\left(u_{1}\right), \ldots, \psi_{m}\left(u_{m}\right)$ can be used to simulate process $\mathbf{P}(t)$. (Nevertheless, case $\phi_{1}\left(u_{1}\right)=\psi_{1}\left(u_{1}\right), \ldots$, $\phi_{m}\left(u_{m}\right)=\psi_{m}\left(u_{m}\right)$ is not excluded.)

In this case, instead of system (1.3), we get the following system of ordinary autonomous differential equations:

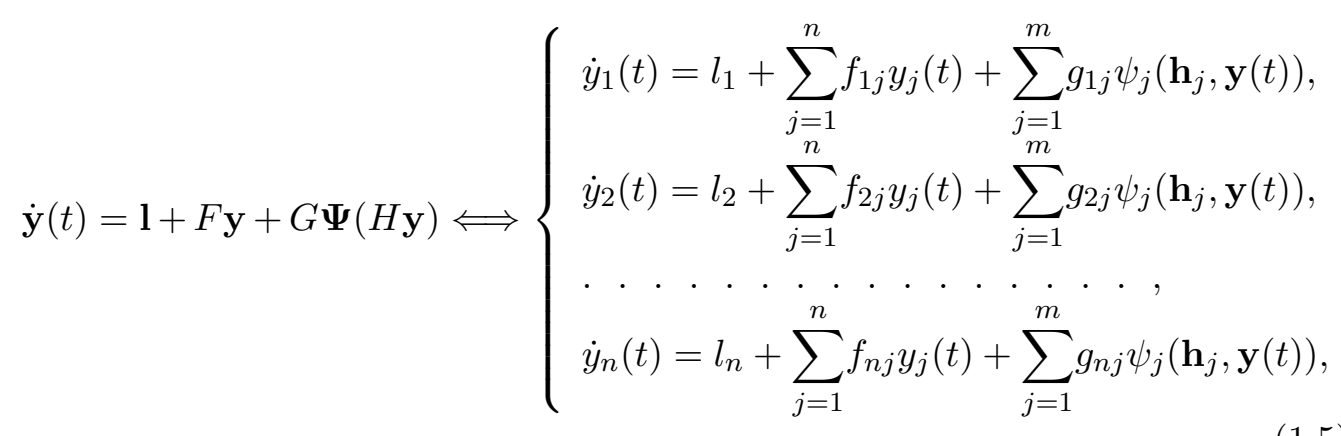

where $\mathbf{\Psi}(\mathbf{v})=\left(\psi_{1}\left(v_{1}\right), \ldots, \psi_{m}\left(v_{m}\right)\right)^{T}$.

Systems (1.3) and (1.5) describe the same dynamic process $\mathbf{P}(t)$. In order to establish (in the sense that will be below) the equivalence of systems (1.3) and (1.5), we introduce the following assumptions:

1) functions $\boldsymbol{\Phi}(\mathbf{u})$ and $\boldsymbol{\Psi}(\mathbf{v})$ are continuous in the interval $(-\infty, \infty)$;

2) $\boldsymbol{\Phi}(\mathbf{0})=\boldsymbol{\Psi}(\mathbf{0})=\mathbf{0}$

3) there exist constants $L_{1}>0, L_{2}>0$, and neighborhood $\mathbb{O}$ of the origin such that $\forall \mathbf{u}, \mathbf{v} \subset \mathbb{O}\|\boldsymbol{\Phi}(\mathbf{u})-\boldsymbol{\Phi}(\mathbf{v})\|_{2}<L_{1}\|\mathbf{u}-\mathbf{v}\|_{2}$ and $\|\mathbf{\Psi}(\mathbf{u})-\boldsymbol{\Psi}(\mathbf{v})\|_{2}<L_{2}\|\mathbf{u}-\mathbf{v}\|_{2}$.

Let the conditions 1) -3 ) be fulfilled. Then, we can assume that there exist the nondegenerate matrices $S \in \mathbb{R}^{n \times n}, T \in \mathbb{R}^{n \times m}$, and $W \in \mathbb{R}^{m \times n}$ such that $\mathbf{x}=S \mathbf{y}, \mathbf{d}=S \mathbf{l}$, and

$$
\left\{\begin{array}{l}
F=S^{-1} A S, G=S^{-1} B T, H=W^{-1} C S \\
\forall \mathbf{s} \in \mathbb{R}^{m} \mathbf{\Psi}(\mathbf{s})=T^{-1} \mathbf{\Phi}(W \mathbf{s}) .
\end{array}\right.
$$

If the mentioned matrices exist, then systems (1.3) and (1.5) are called equivalent.

To fulfill all equivalence conditions (especially (1.6)), it is necessary to indicate the class of functions $\boldsymbol{\Phi}(\mathbf{s})$ (or $\boldsymbol{\Psi}(\mathbf{s})$ ) that satisfy these conditions. One of these classes is the class of piecewise linear functions. (In the theory of neural networks, this class is a well-known class of rectified linear units (ReLU)). In the present time ReLU are standard functions to increase the depth of learning of neural networks. 
Therefore, we will further assume that either the components of vectors $\boldsymbol{\Phi}(\mathbf{s})$, $\boldsymbol{\Psi}(\mathbf{s})$ are piecewise continuous linear functions or these are piecewise continuous nonlinear functions such that $\mathbf{\Phi}(\mathbf{s})=\boldsymbol{\Psi}(\mathbf{s})$ (in this case $T=W=E_{m}$, where $E_{m}$ is the identity matrix of order $m$ ).

A common practice in chaotic time series analysis has been to reconstruct the phase space by utilizing the delay-coordinate embedding technique, and then to compute the dynamical invariants such as fractal dimension of the underlying chaotic set, and its Lyapunov spectrum. As a large body of literature exists on application of the technique of the time series to study chaotic attractors [29] [31], [34], [38], [49], a relatively unexplored issue is its applicability to dynamical systems of differential equations depending on parameters. Our focus will be concentrated on the analysis of influence of parameters of found dynamic system on the behavior of its solutions. These parameters are determined by the structure of the time series (1.1) and choice of approximating functions in the right-hand sides of the obtained system of differential equations.

\subsection{Continuous analog of neural network models}

In recent years, an interesting idea has appeared to interpret a system of ordinary differential equations in the form of a suitable neural network (residual network) $[11,27,51]$. The essence of this idea is as follows.

Consider the following neural network

$$
\mathbf{x}(t+1)=\mathbf{x}(t)+\mathbf{h}(\mathbf{x}(t), \boldsymbol{\Omega}), \mathbf{x}(0)=\mathbf{x}_{0} ; t=1, \ldots, N .
$$

Here $\mathbf{h}(\mathbf{u}, \mathbf{v}): \mathbb{R}^{n} \times \mathbb{R}^{k} \rightarrow \mathbb{R}^{n}$ is a vector of continuous functions, $\Omega: \mathbb{R}^{k} \rightarrow \mathbb{R}^{n}$ is a vector of parameters.

Now we rewrite relation (1.7) in the following form:

$$
\frac{\mathbf{x}(t+1)-\mathbf{x}(t)}{(t+1)-t}=\mathbf{h}(\mathbf{x}(t), \boldsymbol{\Omega})
$$

If we consider function $\mathbf{x}(t)$ as a function of a continuous argument on some interval $\left[\mathbf{x}_{0}, \mathbf{x}_{N}\right]$, then the last equation can be rewritten in the following form:

$$
\frac{\mathbf{x}(t+\Delta t)-\mathbf{x}(t)}{\Delta t}=\mathbf{h}(\mathbf{x}(t), \boldsymbol{\Omega})
$$

If now we direct the number of "layers" $N \rightarrow \infty$ and we assume $\Delta t \rightarrow 0$, then we get the following system of ordinary differential equations

$$
\dot{\mathbf{x}}(t)=\mathbf{h}(\mathbf{x}(t), \boldsymbol{\Omega}), \mathbf{x}(0)=\mathbf{x}_{0},
$$

So we can say that neural network (1.7) is the well-known Euler discretization procedure of system (1.8):

$$
\mathbf{x}(t+\Delta t)-\mathbf{x}(t)=\Delta t \cdot(\mathbf{h}(\mathbf{x}(t), \boldsymbol{\Omega})), \mathbf{x}(0)=\mathbf{x}_{0},
$$




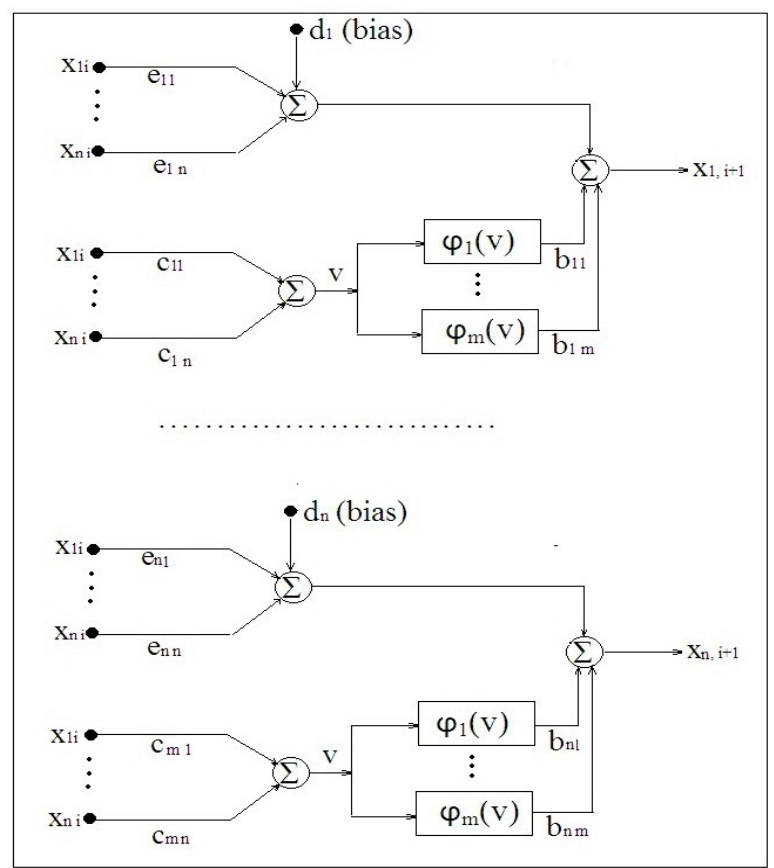

Fig. 1.1. The architecture of the $i$-th layer of the neural network (1.7) for $\mathbf{h}(\mathbf{x}(t), \boldsymbol{\Omega}) \equiv \mathbf{d}+$ $A \mathbf{x}(t)+B \boldsymbol{\Phi}(C \mathbf{x}(t)) ; i=0, \ldots, N$. Here $e_{k j}=a_{k j}$, if $k \neq j$ and $e_{k k}=a_{k k}+1 ; k, j=1, \ldots, n$. All other designations are the same as in (1.3)

where $\Delta t$ is the discretization step.

Besides, sequence (1.7) can be viewed as a neural network with $N-1$ hidden layers, input layer $\mathbf{x}_{0}$ and output layer $\mathbf{x}_{N}$. The architecture of such neural network is determined by the operator $\mathbf{h}(\mathbf{x}(t), \boldsymbol{\Omega})$, and if $\mathbf{h}(\mathbf{x}(t), \boldsymbol{\Omega}) \equiv \mathbf{d}+A \mathbf{x}(t)+$ $B \boldsymbol{\Phi}(C \mathbf{x}(t))$, then an arbitrary hidden layer of this network will have the structure shown in Fig. 1.

Therefore, sequence (1.7) is a neural network model of the process $\mathbf{P}(t)$. In addition, the number of neurons in an arbitrary hidden layer of this network, in which $\mathbf{h}(\mathbf{x}(t), \boldsymbol{\Omega}) \equiv \mathbf{d}+A \mathbf{x}(t)+B \boldsymbol{\Phi}(C \mathbf{x}(t))$, does not exceed $n m[17,26]$.

The problem that is usually considered when modeling process $\mathbf{P}(t)$ is as follows: find parameters $\mathbf{d}, A, B, C$ (with the known activation function $\mathbf{\Phi}(\mathbf{u})$ ) of the neural network that minimize the following loss function:

$$
\sum_{t=1}^{N}\|\mathbf{P}(t)-\mathbf{x}(t)\|^{2} .
$$

Here $\|\mathbf{u}\|$ is a norm of the vector $\mathbf{u}$.

Since the number $n$ is the dimension of the embedding space and it is completely determined by process $\mathbf{P}(t)$, the numbers $N$ and $n m$, which determine the depth and number of neurons in any layer of the neural network, can be arbitrarily 
selected. Thus, there are quite wide opportunities for the neural network modeling of process $\mathbf{P}(t)$.

Now, by analogy with (1.3), we assume that process $\mathbf{P}(t)$ is modeled by neural network (1.7), whose operator has the form $\mathbf{h}(\mathbf{x}(t), \boldsymbol{\Omega}) \equiv \mathbf{l}+F \mathbf{y}(t)+G \mathbf{\Psi}(H \mathbf{y}(t))$. (In this case, we are already talking about defining parameters $1, F, G, H$ with the known activation function $\mathbf{\Psi}(\mathbf{u})$.) Obviously, the equivalence conditions for such neural networks coincide with conditions (1.6). Therefore, the problems of establishing the equivalence of two differential or two neural network models do not differ from each other. In both cases, conditions (1.6) should be used to confirm equivalence. (However, it should be noted that differential and neural network models of the same process, generally speaking, are not equivalent [51].)

In conclusion of this section, we note that the question of which of the modeling methods either using differential equations or neural networks, is more effective as long as it remains open.

For simplicity, we put in system (1.3) $\mathbf{d}=0$. Now we introduce the following control system [50]:

$$
\dot{\mathbf{x}}(t)=A \mathbf{x}+B \mathbf{u}, \mathbf{z}=C \mathbf{x} ; \mathbf{u}=\left(u_{1}, \ldots, u_{m}\right)^{T}, \mathbf{z}=\left(z_{1}, \ldots, z_{m}\right)^{T} .
$$

Then system (1.3) can be viewed as the linear system (1.9) closed by nonlinear feedback

$$
\mathbf{u}=\boldsymbol{\Phi}(\mathbf{z})=\boldsymbol{\Phi}(C \mathbf{x})=\left(\phi_{1}\left(c_{11} x_{1}+\ldots+c_{1 n} x_{n}\right), \ldots, \phi_{m}\left(c_{m 1} x_{1}+\ldots+c_{m n} x_{n}\right)\right)^{T} .
$$

Our main goal is to show how the problem of reconstructing differential equations from known time series can be reduced to the problem of computing invariants for the linear control system (1.9).

Thus, the main postulate that we will implement in this work can be formulated as follows: two different sets of time series (1.2) and (1.4) describe the same dynamic process $\mathbf{P}(t)$ in two different bases of phase space $\mathbb{R}^{n}$; this assumption ensures that the invariants of systems (1.3) and (1.5) are the same.

This article is organized as follows. Section 2 describes the mathematical concepts necessary to solve the equivalence problem. Sections $3-4$ study the actions of algebraic groups on varieties of linear systems. Section 5 gives an algebraic description of the key concept that is used to search for invariants; this is the concept of null-forms. Section 6 is devoted to the analysis of the structural stability of linear systems (small changes in the parameters of the system should not influence on the composition of the invariants of this system). Sections $7-9$ describe rings of invariants and equivalence conditions for linear control systems. The whole Section 10 is devoted to the application of the theory of invariants to the problem of reconstruction of ordinary differential equations from known measurements of the dynamic characteristics of these equations. Here is a practical example of the reconstruction of equations describing the behavior of current and voltage in the contact electric network [7,43]. Finally, some results are summarized in Section 11. 


\section{Mathematical preliminaries}

Consider a linear time-invariant control system whose state equation is

$$
\dot{\mathbf{x}}(t)=\mathbf{A x}(t)+\mathbf{B u}(t), \mathbf{x}(t) \in \mathbb{R}^{n}, \mathbf{u}(t) \in \mathbb{R}^{m},
$$

and an output equation has the form

$$
\mathbf{y}(t)=\mathbf{C x}(t), \mathbf{y}(t) \in \mathbb{R}^{p} .
$$

Here $\mathbb{R}^{n}, \mathbb{R}^{m}, \mathbb{R}^{p}$ are real linear spaces of vector-columns of dimensionalities $n, m, p ; \mathbf{x}(t)=\left(x_{1}(t), \ldots, x_{n}(t)\right)^{T}, u(t)=\left(u_{1}(t), \ldots, u_{m}(t)\right)^{T}$, and $\mathbf{y}(t)=\left(y_{1}(t), \ldots\right.$, $\left.y_{p}(t)\right)^{T}$ are vectors of states, inputs, and outputs; $\mathbf{A}: \mathbb{R}^{n} \rightarrow \mathbb{R}^{n}, \mathbf{B}: \mathbb{R}^{m} \rightarrow$ $\mathbb{R}^{n}, \mathbf{C}: \mathbb{R}^{n} \rightarrow \mathbb{R}^{p}$ are real linear maps of appropriate spaces.

Fix any bases in spaces $\mathbb{R}^{n}, \mathbb{R}^{m}$, and $\mathbb{R}^{p}$; then the triple of operators $\mathbf{A}, \mathbf{B}, \mathbf{C}$ will be represented in the chosen bases by matrices $A \in \mathbb{R}^{n \times n}, B \in \mathbb{R}^{n \times m}$, and $C \in \mathbb{R}^{p \times n}$. Further, we will adhere only to these denotations.

Denote by $\mathbb{S}=\mathbb{R}^{p \times n} \times \mathbb{R}^{n \times n} \times \mathbb{R}^{n \times m}$ a direct product of spaces $\mathbb{R}^{p \times n}, \mathbb{R}^{n \times n}$, and $\mathbb{R}^{n \times m}$. Then system $(2.1),(2.2)$ can be uniquely represented by the triple of matrices $(C, A, B) \in \mathbb{S}$.

Let $\mathbb{G} L(q, \mathbb{R})$ be a complete linear group of all square invertible matrices of sizes $q \times q$ with elements from the field of real numbers $\mathbb{R}$.

Introduce the direct product

$$
\begin{gathered}
\mathbb{G} L=\mathbb{G} L(n, \mathbb{R}) \times \mathbb{G} L(m, \mathbb{R}) \times \mathbb{G} L(p, \mathbb{R}) \\
=\{(S, T, W) \mid S \in \mathbb{G} L(n, \mathbb{R}), T \in \mathbb{G} L(m, \mathbb{R}), W \in \mathbb{G} L(p, \mathbb{R})\} .
\end{gathered}
$$

We also introduce into system (2.1),(2.2) new variables $\mathbf{z} \in \mathbb{R}^{n}, \mathbf{v} \in \mathbb{R}^{m}$, and $\mathbf{h} \in \mathbb{R}^{p}$ under the formulas: $\mathbf{x}(t)=S \mathbf{z}(t), \mathbf{u}(t)=T \mathbf{v}(t)$, and $\mathbf{y}(t)=W \mathbf{h}(t)$, where $S \in \mathbb{G} L(n, \mathbb{R}), T \in \mathbb{G} L(m, \mathbb{R})$, and $W \in \mathbb{G} L(p, \mathbb{R})$. Then we obtain the following operation $\mathbb{G} L: \mathbb{S} \rightarrow \mathbb{S}$ of indicated group on space $\mathbb{S}$ :

$$
\begin{gathered}
\forall(C, A, B) \in \mathbb{S} \text { and } \forall(S, T, W) \in \mathbb{G} L, \\
(S, T, W) \circ(C, A, B)=\left(W^{-1} C S, S^{-1} A S, S^{-1} B T\right) \in \mathbb{S} .
\end{gathered}
$$

Let $\mathbf{s}=(C, A, B) \in \mathbb{S}$ and $\mathbf{g}=(S, T, W) \in \mathbb{G} L$ be an arbitrary elements of corresponding sets.

Definition 2.1. A polynomial $f(\mathbf{s})$ is called an invariant of weight $\mathbf{l}=\left(l_{S}, l_{T}, l_{W}\right)$ of group $\mathbb{G} L$ for system $(2.1),(2.2)$ if

$$
f(\mathbf{g} \circ \mathbf{s})=(\operatorname{det} S)^{l_{S}}(\operatorname{det} T)^{l_{T}}(\operatorname{det} W)^{l_{W}} \times f(\mathbf{s}), \quad \forall \mathbf{g} \in \mathbb{G} L \text { and } \forall \mathbf{s} \in \mathbb{S},
$$

where $l_{S}, l_{T}$, and $l_{W}$ are some integers. The invariant $f(\mathbf{s})$ of weight $\mathbf{l}=\left(l_{S}, l_{T}, l_{W}\right)$ $=(0,0,0)$ is called absolute; otherwise the invariant $f(\mathbf{s})$ is called relative $[35,44]$. 
Notice that the problem of any classification of some set of objects (for example, the set of systems (2.1), (2.2)) implies a decomposition of this set on classes of identical (in a certain sense) elements. One of the most widespread methods of such decomposition is a description of system (2.1), (2.2) with the help of functions not depending on coordinate presentation of system (2.1), (2.2). This description is usually called invariant. Sometimes the invariant description of system (2.1), (2.2) is called an invariant (or algebraic) analysis.

The problem of invariant analysis of system (2.1),(2.2) with respect to action (2.3) was most in detail studied for the case $T=E_{m}$ and $W=E_{p}$, where $E_{m}$ and $E_{p}$ are identity matrices of degrees $m$ and $p$. In this case the action (2.3) is called an action of similarity. In one or another form classification questions of linear control systems with respect to the action of similarity, their invariants and canonical forms were studied by many authors (see, for example, C. I. Byrnes and N. E. Hurt [9], M. Hazewinkel [18, 20], M. Hazewinkel and C. Martin [19], R. E. Kalman [28], A. Tannenbaum $[45,46])$. We also note that in the book of D. Mumford and J. Fogarty [35] the tasks of invariant theory, directly relating to the classification questions of linear control systems, were considered. The topology problems of classification of linear control systems with respect to the action of similarity in detail were studied in articles of D. F. Delchamps [12], P. A. Fuhrmann and U. Helmke [14], and U. Helmke [21] - [23].

Note that a main attention of specialists on the mathematical system theory attract problems of invariant analysis (using geometric invariant theory) connected with the action of similarity. These problems are following: the finding of good canonical forms for linear systems, a computation of their invariants, a description of regular and stable systems, and also a construction of moduli spaces as quotients of algebraic varieties under algebraic group actions. It should be said that the indicated problems were considered with different positions by A. Tannenbaum [46, 47], S. Friedland [13], V. G. Lomadze [32], J. Rosenthal [41], W. Manthey and U. Helmke [33], M. Bader [1]. In our opinion the most complete solutions of indicated problems was got in [1]. In this work with the help of geometric invariant theory and methods of theory quivers compactifications of moduli spaces of linear dynamical systems were derived.

Except for the classification questions of linear control systems, invariant theory is widely used in the problem of output feed-back design both for linear and bilinear control systems (see, for examples, papers [2] - [6], [38], [39], [41, 42], and [50]). Here it should be said that in article [5] it was succeeded to get the constructive solution of output feed-back design problem for system (2.1), (2.2) in the case $m p>n$.

Consider two systems: $\mathbf{s}_{1}=\left(C_{1}, A_{1}, B_{1}\right) \in \mathbb{S}_{\text {open }} \subset \mathbb{S}$ and $\mathbf{s}_{2}=\left(C_{2}, A_{2}, B_{2}\right) \in$ $\mathbb{S}_{\text {open }} \subset \mathbb{S}$, where $\mathbb{S}_{\text {open }}$ is an open subset in $\mathbb{S}$.

Equivalence problem. It is necessary to find the set $\mathbb{S}_{\text {open }} \subset \mathbb{S}$ and to build the finite set of invariants $\mathbf{f}_{1}(\mathbf{s}), \ldots, \mathbf{f}_{k}(\mathbf{s})$ (absolute and relative) of group $\mathbb{G} L$ such that $\forall \mathbf{s}_{1}, \mathbf{s}_{2} \in \mathbb{S}_{\text {open }}$ from the conditions $\mathbf{f}_{1}\left(\mathbf{s}_{1}\right)=\mathbf{f}_{1}\left(\mathbf{s}_{2}\right), \ldots, \mathbf{f}_{k}\left(\mathbf{s}_{1}\right)=\mathbf{f}_{k}\left(\mathbf{s}_{2}\right)$ it follows 
that there exists the element $\mathbf{g}=(S, T, W) \in \mathbb{G} L$ such that

$$
A_{2}=S^{-1} A_{1} S, B_{2}=S^{-1} B_{1} T, C_{2}=W^{-1} C_{1} S .
$$

It should be said that in the present time there is a vast literature devoted to invariant theory and its applications to different tasks of mathematics, mechanics, physics, and control theory. However almost in all known manuscripts invariant theory is considered as part of algebraic geometry and theory of representations of groups. In addition, these treatises are intended for professional mathematicians and ineligible for specialists in applied system theory. It superfluously burdens an application of invariant theory to linear control systems, which this article is devoted. In this connection, basic results of the present work will be presented in terms of ordinary linear spaces, matrices and determinants. One of aims of the article is bringing of the solution of equivalence problem for linear control systems to such level, as it is done at description of invariant properties of a characteristic polynomial of square matrix.

In the present time the equivalence problem is not yet solved completely. In this connection we will solve this problem in a few stages.

On the first from these stages we study the simplified variant of equivalence problem, for which equation (2.2) in system (2.1), (2.2) is absent. In this case we suppose that the group $\mathbb{G} L=\mathbb{G} L(n, \mathbb{R}) \times \mathbb{G} L(m, \mathbb{R})$ and the space $\mathbb{S}=\mathbb{R}^{n \times n} \times$ $\mathbb{R}^{n \times m}$.

Now we change the base field $\mathbb{R}$ and the group $\mathbb{G} L$ by the base field $\mathbb{C}$ and the special linear group $\mathbb{S} L=\mathbb{S} L(n, \mathbb{C}) \times \mathbb{S} L(m, \mathbb{C})$ saving its action $(2.3)$ on the complex space $\mathbb{S}=\mathbb{C}^{n \times n} \times \mathbb{C}^{n \times m}$.

In this case all relative invariants of group $\mathbb{G} L$ become absolute invariants of group $\mathbb{S} L$. This circumstance allows us to use the Hilbert-Mumford theory $[8,35]$ for description of all invariants of group $\mathbb{S} L$ with respect to action (2.3). Therefore in further, all absolute and relative invariants we will call simply invariants.

Denote by $\mathbb{C}[\mathbb{S}]^{\mathbb{S} L}$ a ring of all invariants of group $\mathbb{S} L$ with respect to action (2.3) $[35,44]$. A search problem of invariants can be essentially simplified if we will take advantage of the following concept [35].

Definition 2.2. (See [35]). The element $\mathbf{w} \in \mathbb{S}$ is called a null-form if for an arbitrary non-constant invariant $I(\cdot) \in \mathbb{C}[\mathbb{S}]^{\mathbb{S} L} \quad I(\mathbf{w})=0$.

Let $\mathbf{w}$ be an element of $\mathbb{S}$ and let $\mathbb{H}$ be an arbitrary subgroup of $\mathbb{S} L$. Denote by $\mathbb{O}^{\mathbb{H}}(\mathbf{w}) \subset \mathbb{S}$ an orbit of the point w with respect to action (2.3) of group $\mathbb{H}$. Let $\overline{\mathbb{O}^{\mathbb{H}}(\mathbf{w})} \subset \mathbb{S}$ be the closure of $\mathbb{O}^{\mathbb{H}}(\mathbf{w})$ in $\mathbb{S}$.

The following theorem is a basic instrument for search of null-forms.

Theorem 2.1. (See [35]). The element $\mathbf{w} \subset \mathbb{S}$ is the null-form if and only if there exists a multiplicative one-parameter subgroup $\mathbb{H} \subset \mathbb{S} L$ such that the point $\mathbf{0}=\left(0^{n \times n} \times 0^{n \times m}\right) \in \overline{\mathbb{O}^{H}}(\mathbf{w})$. 


\subsection{Null-formes of space $\mathbb{S}$ for system (2.1)}

Further, for system (2.1) we will use the designation $(A, B)$, where $A \in \mathbb{C}^{n \times n}$, $B \in \mathbb{C}^{n \times m}$. We will also call the system $(A, B)$ by a system of type $(n, m)$, where numbers $n$ and $m$ are dimensions of the state space and input space. In addition, we will designate the state space and input space by $\mathbb{X} \equiv \mathbb{C}^{n}$ and $\mathbb{U} \equiv \mathbb{C}^{m}$.

Without loss of generality, it is possible to consider that $\operatorname{rank} B=m$. In addition, we will also assume that $n>m$, since in control theory this case is most important.

The following concept in linear control theory is fundamental.

Definition 2.3. (See [28]). The system $(A, B)$ is called complete controllable if

$$
\mathbb{X}=\operatorname{span}\left\{B(\mathbb{U}), A B(\mathbb{U}), \ldots, A^{n-1} B(\mathbb{U})\right\} .
$$

Let $\mathbb{X}_{1} \subset \mathbb{X}$ be an invariant subspace in $\mathbb{X}$ with respect to the following action of operator $A: A \mathbb{X}_{1} \subset \mathbb{X}_{1}[15]$.

We put $\mathbb{U}_{1}=B^{-1}\left(\mathbb{X}_{1} \cap B(\mathbb{U})\right) \subset \mathbb{U}$, where $B^{-1}(\mathbb{X})$ is a complete prototype of operator $B: \mathbb{U} \rightarrow \mathbb{X}[15]$.

Denote by $\left.G\right|_{\mathbb{L}}$ the restriction of operator $G: \mathbb{C}^{k} \rightarrow \mathbb{C}^{r}$ to subspace $\mathbb{L} \subset \mathbb{C}^{k}$ [15]. (Here $k$ and $r$ are natural numbers.)

Definition 2.4. The pair of operators $\left(\left.A\right|_{\mathbb{X}_{1}},\left.B\right|_{\mathbb{U}_{1}}\right)$, where $\left.A\right|_{\mathbb{X}_{1}}$ and $\left.B\right|_{\mathbb{U}_{1}}$ are restrictions of operators $A$ and $B$ to subspaces $\mathbb{X}_{1}$ and $\mathbb{U}_{1}$ of dimensionalities $n_{1}$ and $m_{1}$, is called a subsystem of type $\left(n_{1}, m_{1}\right)$ of system $(A, B)$.

Definition 2.5. The pair of operators $\left(\left.A\right|_{\mathbb{X} / \mathbb{X}_{1}},\left.B\right|_{\mathbb{U} / \mathbb{U}_{1}}\right)$, where $\left.A\right|_{\mathbb{X} / \mathbb{X}_{1}}$ and $\left.B\right|_{\mathbb{U} / \mathbb{U}_{1}}$ are restrictions of operators $A$ and $B$ to factor-spaces $\mathbb{X} / \mathbb{X}_{1}$ and $\mathbb{U} / \mathbb{U}_{1}$ of dimensionalities $n-n_{1}$ and $m-m_{1}$, is called a factor-system of type $\left(n-n_{1}, m-m_{1}\right)$ of system $(A, B)$ on the subsystem $\left(\left.A\right|_{\mathbb{X}_{1}},\left.B\right|_{\mathbb{U}_{1}}\right)$.

Theorem 2.2. Let $(A, B)$ be a system of type $(n, m), n>m$. Then the system $(A, B)$ is a null-form if and only if the operator $A$ is nilpotent and $(A, B)$ contains the subsystem $\left(\left.A\right|_{\mathbb{X}_{1}},\left.B\right|_{\mathbb{U}_{1}}\right)$ of type $\left(n_{1}, m_{1}\right), n_{1} \geq m_{1}$, such that

$$
\frac{n_{1}}{m_{1}}<\frac{n}{m} \text {. }
$$

Proof. (a1) It is known $[35,44]$ that in the suitable bases of spaces $\mathbb{C}^{n}$ and $\mathbb{C}^{m}$ the one-parametric group $\mathbb{H}$ can be represented by the group of diagonal matrices:

$$
\mathbb{H}=\mathbb{H}_{1} \times \mathbb{H}_{2}=\left(\begin{array}{ccc}
t^{-\alpha_{1}} & \cdots & 0 \\
\vdots & \ddots & \vdots \\
0 & \cdots & t^{-\alpha_{n}}
\end{array}\right) \times\left(\begin{array}{ccc}
t^{-\beta_{1}} & \cdots & 0 \\
\vdots & \ddots & \vdots \\
0 & \cdots & t^{-\beta_{m}}
\end{array}\right)
$$

where $t$ is a real parameter, and real numbers $\alpha_{1}, \alpha_{2}, \ldots, \alpha_{n}, \beta_{1}, \beta_{2}, \ldots, \beta_{m}$ are satisfied to the following restrictions:

$$
\alpha_{1}+\alpha_{2}+\ldots+\alpha_{n}=0, \beta_{1}+\beta_{2}+\ldots+\beta_{m}=0 .
$$


Note that if the group $\mathbb{H}$ acts on the system $(A, B)$ by rule (2.3), then the elements of matrices $A$ and $B$ will be transformed on formulas:

$$
a_{i k} \rightarrow a_{i k} t^{\alpha_{i}-\alpha_{k}}, b_{i j} \rightarrow b_{i j} t^{\alpha_{i}-\beta_{j}} ; i, k=1, \ldots, n ; j=1, \ldots, m .
$$

The trivial system $(0,0) \subset \mathbb{C}^{n \times n} \times \mathbb{C}^{n \times m}$ lies in the closure of orbit $O^{\mathbb{H}}(A, B)$ of system $(A, B)$ if and only if there exist the following limits:

$$
\lim _{t \rightarrow \infty} \mathbb{H}_{1}^{-1} A \mathbb{H}_{1}=0, \lim _{t \rightarrow \infty} \mathbb{H}_{1}^{-1} B \mathbb{H}_{2}=0 .
$$

(a2) Both last limits take place if and only if there is a nontrivial joint solution of equalities (2.6) and the following system of inequalities and equalities:

$$
\begin{gathered}
b_{i j}=0 \text { or } \alpha_{i}<\beta_{j}, i=1, \ldots, n ; j=1, \ldots, m ; \\
a_{i i}=0,1 \leq i \leq n \\
a_{i k}=0 \text { or } \alpha_{i}<\alpha_{k}, 1 \leq k<i \leq n .
\end{gathered}
$$

First, we suppose that $b_{i m}=0$ for $i=1, \ldots, n$. Then inequalities (2.7) replace by inequalities:

$$
\alpha_{i}<\beta_{j}, i=1, \ldots, n ; j=1, \ldots, m-1 .
$$

Further, inequalities (2.7) are shown that numbers $\alpha_{i}$ (or $\beta_{j}$ ) can not have one sign. Therefore, we will assume that, for example, $\alpha_{n}>0$. Now we choose numbers $\alpha_{1}, \ldots, \alpha_{n}$, which satisfy to conditions (2.7) and $\alpha_{n}>0$.

Assume that the number $\beta_{i}$ is given so that inequalities (2.10) will be fulfilled. Then the number $\beta_{m}$ can be computed on formula (2.6): $\beta_{m}=-\beta_{1}-\ldots-\beta_{m-1}$.

From here it follows that the system of inequalities (2.10) is solvable. Consequently, systems $(2.7)-(2.8)$ are also solvable. From here it follows that if $n<m$, then taking into account conditions (2.9), which determines the nilpotent matrix $A$, all systems $(A, B)$ of type $(n, m)$ are null-forms. (By transformations from the group $\mathbb{S} L$ in the matrix $B$, it is possible to derive one zero column.)

If $n=m$ and $\operatorname{det} B \neq 0$, then the system $(A, B)$ is not a null-form; if $\operatorname{det} B=0$, then it can be reduced to the case $n<m$. Consequently, at $n>m$ the search of null-forms can be taken to the search of systems $(A, B)$, for which $\operatorname{rank} B=m$.

Further, changing bases in the spaces $\mathbb{X}$ and $\mathbb{U}$, it is possible to obtain that at $n_{1} \leq i \leq n$ and $1 \leq j \leq m_{1}$ in the matrix $B$ the elements $b_{i j}=0$. In this case inequalities (2.7) can be transformed in inequalities:

$$
\begin{gathered}
\alpha_{i}<\beta_{j}, i=1, \ldots, n_{1} ; j=1, \ldots, m ; \\
\alpha_{i}<\beta_{j}, i=n_{1}+1, \ldots, n ; j=m_{1}+1, \ldots, m .
\end{gathered}
$$

Summing inequalities (2.11) $n_{1}$ times and taking into account the first from equalities (2.6), we get

$$
-\left(\alpha_{n_{1}+1}+\ldots+\alpha_{n}\right)<n_{1} \beta_{j}, j=1, \ldots, m .
$$


It is like easily to get from those inequalities (2.11) that

$$
m \alpha_{i}<\beta_{1}+\ldots+\beta_{m}=0, i=1, \ldots, n_{1} .
$$

Now summing inequalities (2.13) up to $j=m_{1}$ and taking into account the second from equalities (2.6), we have

$$
-m_{1}\left(\alpha_{n_{1}+1}+\ldots+\alpha_{n}\right)<-n_{1}\left(\beta_{m_{1}+1}+\ldots+\beta_{m}\right) .
$$

From here we get relation, which is equivalent to the following inequality:

$$
\left(\alpha_{n_{1}+1}+\ldots+\alpha_{n}\right)>\frac{n_{1}}{m_{1}}\left(\beta_{m_{1}+1}+\ldots+\beta_{m}\right) .
$$

Having fulfilled similar computations for system (2.12), we get

$$
\left(\alpha_{n_{1}+1}+\ldots+\alpha_{n}\right)>\frac{n-n_{1}}{m-m_{1}}\left(\beta_{m_{1}+1}+\ldots+\beta_{m}\right) .
$$

A comparison of inequalities (2.15) and (2.16) results in the double inequality

$$
\frac{n-n_{1}}{m-m_{1}}\left(\beta_{m_{1}+1}+\ldots+\beta_{m}\right)>\left(\alpha_{n_{1}+1}+\ldots+\alpha_{n}\right)>\frac{n_{1}}{m_{1}}\left(\beta_{m_{1}+1}+\ldots+\beta_{m}\right) .
$$

(a3) From (2.14) and first from equalities (2.6), we have $\alpha_{n_{1}+1}+\ldots+\alpha_{n}>0$.

Assume that $\beta_{m_{1}+1}+\ldots+\beta_{m}>0$. Then the solvability of inequality (2.17) is possible only in the case $\left(n-n_{1}\right) /\left(m-m_{1}\right)>n_{1} / m_{1}$ (or $\left.n / m>n_{1} / m_{1}\right)$. If the case $\beta_{m_{1}+1}+\ldots+\beta_{m}<0$ takes place, then the restriction $\left(n-n_{1}\right) /\left(m-m_{1}\right)<0$ must be valid. This inequality results in inequalities: either $n>n_{1}$ and $m<m_{1}$ or $n<n_{1}$ and $m>m_{1}$ that it is impossible by virtue of the conditions $n>n_{1}$ and $m>m_{1}$.

Further, from conditions (2.9), which determine the form of the matrix $A$, it follows that $n_{1}$ is the dimension of the invariant with respect to operator $A$ subspace $\mathbb{X}_{1}$, which contains subspace $B\left(\mathbb{U}_{1}\right) \subset \mathbb{X}_{1}$ spanned on $m_{1}$ first columns of the matrix $B$.

Suppose opposite: if in the space $B(\mathbb{U})$ there doesn't exist of the subspace $B\left(\mathbb{U}_{1}\right)$ such that

$$
B\left(\mathbb{U}_{1}\right) \subset \mathbb{X}_{1}=\operatorname{span}\left\{B\left(\mathbb{U}_{1}\right), A B\left(\mathbb{U}_{1}\right), \ldots, A^{n-1} B\left(\mathbb{U}_{1}\right)\right\}
$$

and $\left(\operatorname{dim} \mathbb{X}_{1}\right) /\left(\operatorname{dim} \mathbb{U}_{1}\right)=n_{1} / m_{1}<n / m$, then system $(A, B)$ is the null-form. Really, if $n_{1} / m_{1} \geq n / m$, then $\left(n-n_{1}\right) /\left(m-m_{1}\right) \leq n_{1} / m_{1}$ and under the condition $\alpha_{n_{1}+1}+\ldots+\alpha_{n}>0$ inequality (2.17) is incorrect. Consequently, the system of restrictions $(2.7)-(2.9)$ is incompatible. The proof is finished.

Notice that the nilpotency of matrix $A[15]$ is necessary for an equality to zero of invariants of system $(A, B)$ depending only on $A$. The equality to zero of invariants of system $(A, B)$ depending on $B$ is got without the use of concept of nilpotency. Thus, the following statement is obvious. 
Corollary. Let $A$ be an arbitrary matrix of order $n$. Then under condition (2.5) of Theorem 2.2, all invariants of the system $(A, B)$ depending on $B$ are equal to zero.

\section{Stabilizer of system $(A, B)$}

Let $\psi(\lambda)$ be a minimal polynomial of matrix $A \in \mathbb{C}^{n \times n}$ [15]. (It means that polynomial $\psi(\lambda)$ is the nonzero polynomial of the least degree $l \leq n$ such that $\psi(A)=0$.)

Assume that a nonzero vector from $B(\mathbb{U})$ has a minimal polynomial $f_{1}(\lambda)$. Suppose also that this polynomial is the polynomial of the least degree among degrees of minimal polynomials of all nonzero vectors from $B(\mathbb{U})$.

Denote by $\mathbb{L}_{1}$ the set all nonzero vectors from $B(\mathbb{U})$, which have the same minimal polynomial $f_{1}(\lambda)$. Add the zero vector to the set $\mathbb{L}_{1}$. Denote the newly obtained set with the same symbol $\mathbb{L}_{1}$.

Lemma 3.1. $\mathbb{L}_{1}$ is the linear subspace in $B(\mathbb{U})$.

Proof. Let $\mathbb{U}_{1}=B^{-1}\left(\mathbb{L}_{1}\right)$ be a complete prototype of space $\mathbb{L}_{1}$ in $\mathbb{U}$ with respect to the action of operator $B$. Then it is clear that subspace

$$
\mathbb{X}_{1}=\operatorname{span}\left\{B\left(\mathbb{U}_{1}\right), A B\left(\mathbb{U}_{1}\right), \ldots, A^{n-1} B\left(\mathbb{U}_{1}\right)\right\}
$$

is invariant with respect to the action of operator $A$ and it minimal polynomial coincides with $f_{1}(\lambda)$.

Let $f_{1}(\lambda) \neq \psi(\lambda)$. Consider the factor-system $\left(\left.A\right|_{\mathbb{X} / \mathbb{X}_{1}},\left.B\right|_{\mathbb{U} / \mathbb{U}_{1}}\right)$. Then it is possible to find in the factor-space $\left(B(\mathbb{U})+\mathbb{X}_{1}\right) / \mathbb{X}_{1}$ the subspace $\mathbb{L}_{2} / \mathbb{X}_{1}$, all nonzero vectors of which have the same minimal polynomial $f_{2}(\lambda)\left(\bmod \mathbb{X}_{1}\right)[15]$. In addition, the degree of this polynomial will be minimum among degrees of all minimal polynomials, which can have nonzero vectors from $\left(B(\mathbb{U})+\mathbb{X}_{1}\right) / \mathbb{X}_{1}$.

Now we build the invariant with respect to action of operator $A$ subspace

$$
\mathbb{X}_{2}=\operatorname{span}\left\{B\left(\mathbb{U}_{2}\right), A B\left(\mathbb{U}_{2}\right), \ldots, A^{n-1} B\left(\mathbb{U}_{2}\right)\right\} \subset \mathbb{X} .
$$

Here $\mathbb{U}_{2}=B^{-1}\left(\mathbb{L}_{2}\right)$ is the complete prototype of space $\mathbb{L}_{2}$ in $\mathbb{U}$ with respect to the action of operator $B$. If $\mathbb{X}_{2} \neq \mathbb{X}$, then we consider the factor-system $\left(\left.A\right|_{\mathbb{X} / \mathbb{X}_{2}},\left.B\right|_{\mathbb{U} / \mathbb{U}_{2}}\right)$, where $\mathbb{U}_{2}=B^{-1}\left(\mathbb{L}_{2}\right)$ is the complete prototype of subspace $\mathbb{L}_{2}$ in $\mathbb{U}$ with respect to the action of operator $B$, and so on. By virtue of finite dimensionality of the spaces $\mathbb{X}$ and $\mathbb{U}$ the indicated procedure will be finished on some stage $r$ :

$$
\mathbb{U}_{r}=\mathbb{U}, \quad \mathbb{X}_{r}=\mathbb{X}=\operatorname{span}\left\{B\left(\mathbb{U}_{r}\right), A B\left(\mathbb{U}_{r}\right), \ldots, A^{n-1}\left(\mathbb{U}_{r}\right)\right\} .
$$

Thus, we have the row of embedded in each other subsystems

$$
(0,0) \subset\left(\left.A\right|_{\mathbb{X}_{1}},\left.B\right|_{\mathbb{U}_{1}}\right) \subset\left(\left.A\right|_{\mathbb{X}_{2}},\left.B\right|_{\mathbb{U}_{2}}\right) \subset \ldots \subset\left(\left.A\right|_{\mathbb{X}_{r}},\left.B\right|_{\mathbb{U}_{r}}\right)=(A, B)
$$


such that any factor-system of this row possesses the following property: the state space $\mathbb{X}_{i} / \mathbb{X}_{i-1}$ of system $\left(\left.A\right|_{\mathbb{X}_{i} / \mathbb{X}_{i-1}},\left.B\right|_{\mathbb{U}_{i} / \mathbb{U}_{i-1}}\right)$ is generated by the factor-space $\mathbb{L}_{i} / \mathbb{X}_{i-1} \subset\left(B(\mathbb{U})+\mathbb{X}_{i}\right) / \mathbb{X}_{i-1}$, all nonzero vectors of which have the same minimal polynomial $f_{i}(\lambda)\left(\bmod \mathbb{X}_{i-1}\right), i=1, \ldots, r$.

Definition 3.1. (See [20]). The maximal subgroup $\operatorname{Stab}_{\mathbb{S} L}(A, B)$ of group $\mathbb{S} L$ is given by the conditions

$$
\mathbb{S t a b}_{\mathbb{S} L}(A, B)=\{(S, T) \in \mathbb{S} L \mid A S=S A, B T=S B\}
$$

is called a stabilizer of system $(A, B)$.

Lemma 3.2. Let $(S, T) \in \mathbb{S t a b}_{\mathbb{S} L}(A, B)$ be an arbitrary element and $\left(\left.A\right|_{\mathbb{X}_{i}},\left.B\right|_{\mathbb{U}_{i}}\right)$ be an arbitrary system of row (3.1). Then the following inclusion takes place:

$$
(S, T) \cdot\left(\left.A\right|_{\mathbb{X}_{i}},\left.B\right|_{\mathbb{U}_{i}}\right) \in\left(\left.A\right|_{\mathbb{X}_{i}},\left.B\right|_{\mathbb{U}_{i}}\right), i=1, \ldots, r .
$$

Proof. We prove justice (3.1) for $i=1$. Lemma 3.1 asserts that the subspace $\mathbb{L}_{1}$ is uniquely. Then from the definition of stabilizer we get that $S B\left(\mathbb{U}_{1}\right)=$ $\mathbb{U}_{1} \subset B T(\mathbb{U}) \subset B(\mathbb{U})$ and, by virtue of uniqueness of $B\left(\mathbb{U}_{1}\right)$ in $B(\mathbb{U})$, we have $S B\left(\mathbb{U}_{1}\right) \subset B\left(\mathbb{U}_{1}\right)$. Further, from the uniqueness $B\left(\mathbb{U}_{1}\right)$ and that $\mathbb{U}_{1}=B^{-1}\left(\mathbb{L}_{1}\right)$ is the complete prototype $\mathbb{L}_{1}$ in $\mathbb{U}$, it follows that the subspace $\mathbb{U}_{1}$ is a unique in $\mathbb{U}$. It means that $T\left(\mathbb{U}_{1}\right) \subset \mathbb{U}_{1}$. In addition, we have

$$
S\left(\mathbb{X}_{1}\right)=S \sum_{i=1}^{n-1} A^{i} B\left(\mathbb{U}_{1}\right)=\sum_{i=1}^{n-1} A^{i} S B\left(\mathbb{U}_{1}\right)=\sum_{i=1}^{n-1} A^{i} B T\left(\mathbb{U}_{1}\right) \subset \sum_{i=1}^{n-1} A^{i} B\left(\mathbb{U}_{1}\right) \subset \mathbb{X}_{1} .
$$

The proof for case $i=1$ is finished.

Now we lead an induction on $i$. Assume that for some $i<r$

$$
(S, T) \cdot\left(\left.A\right|_{\mathbb{X}_{i}},\left.B\right|_{\mathbb{U}_{i}}\right) \in\left(\left.A\right|_{\mathbb{X}_{i}},\left.B\right|_{\mathbb{U}_{i}}\right) .
$$

Consider the factor-system $\left(\left.A\right|_{\mathbb{X}_{i} / \mathbb{X}_{i-1}},\left.B\right|_{\mathbb{U}_{i} / \mathbb{U}_{i-1}}\right)$. Then the proof of inclusion (3.2) at $i=1$ word for a word is carried on the proof of inclusion

$$
(S, T) \cdot\left(\left.A\right|_{\mathbb{X}_{i} / \mathbb{X}_{i-1}},\left.B\right|_{\mathbb{U}_{i} / \mathbb{U}_{i-1}}\right) \in\left(\left.A\right|_{\mathbb{X}_{i} / \mathbb{X}_{i-1}},\left.B\right|_{\mathbb{U}_{i} / \mathbb{U}_{i-1}}\right) .
$$

Taking into account the supposition of induction, we get the inclusion $(S, T)$. $\left(\left.A\right|_{\mathbb{X}_{i+1}},\left.B\right|_{\mathbb{U}_{i+1}}\right) \in\left(\left.A\right|_{\mathbb{X}_{i+1}},\left.B\right|_{\mathbb{U}_{i+1}}\right)$. It completes the proof of Lemma 3.2.

Lemma 3.3. Let $(A, B)$ be a complete controllable system of type $(n, m), n \geq$ $m, \operatorname{rank} B=m$, and all nonzero vectors of space $B(\mathbb{U})$ have the same minimal polynomial $f(\lambda)$. Then $\mathbb{S t a b}_{\mathbb{S} L}(A, B) \cong \mathbb{S} L(m, \mathbb{C})$.

Proof. We prove that system $(A, B)$ is the direct sum of $m$ copies the irreducible subsystems. (We remind that the system $(A, B)$ is called irreducible if it contains only trivial subsystems $(0,0)$ and $(A, B)[44]$.) 
At $m=1$ the assertion of Lemma 3.3 is obvious. Assume that it is correctly for all $k \leq m-1$. Now we have to prove that $(A, B)$ is the direct sum of equivalent irreducible subsystems for $k=m$.

Let $l=\operatorname{deg} f(\lambda)$. Choose in $B(\mathbb{U})$ an arbitrary base $b_{1}, \ldots, b_{m}$. Denote by the symbol $\mathbb{X}_{i}=\left\{b_{i}, A b_{i} \ldots, A^{l-1} b_{i}\right\} \subset \mathbb{X}$ a cyclic with respect to action of operator $A$ subspace in $\mathbb{X}[15]$. (It is clear that $\operatorname{dim} \mathbb{X}_{i}=l, i=1, \ldots, m$.)

Since the system $(A, B)$ is the complete controllable, then we have $\mathbb{X}=\mathbb{X}_{1}+$ $\ldots+\mathbb{X}_{m}$. On the supposition of induction the last sum can be rewritten as $\mathbb{X}=$ $\mathbb{X}_{1} \oplus \ldots \oplus \mathbb{X}_{m-1}+\mathbb{X}_{m} \equiv \tilde{\mathbb{X}}+\mathbb{X}_{m}$. We will prove that this sum is direct.

Assume that the space $\mathbb{P} \equiv \tilde{\mathbb{X}} \cap \mathbb{X}_{m} \neq \emptyset$. It is clear that the space $\mathbb{P}$ is a proper cyclic subspace in $\mathbb{X}_{m}$ and $\tilde{\mathbb{X}}$.

Let $h(\lambda)$ be the minimal polynomial of space $\mathbb{P}$ (note that $\operatorname{deg} h(\lambda)<\operatorname{deg} f(\lambda)$ ). In this case there exists the vector $\alpha_{1} b_{1}+\ldots+\alpha_{m-1} b_{m-1} \in \tilde{\mathbb{X}}$ and the polynomial $g(\lambda)=f(\lambda) / h(\lambda)$ such that $g(A)\left(\alpha_{1} b_{1}+\ldots+\alpha_{m-1} b_{m-1}\right) \in \mathbb{P}$, and $g(A) b_{m} \in \mathbb{P}$.

Here two following cases are possible.

(i) $g(A)\left(\alpha_{1} b_{1}+\ldots+\alpha_{m-1} b_{m-1}\right)=g(A)\left(\alpha_{m} b_{m}\right)$. Then the polynomial $g(\lambda)$ is minimal for the nonzero vector $\alpha_{1} b_{1}+\ldots+\alpha_{m-1} b_{m-1}-\alpha_{m} b_{m}$. Since $\operatorname{deg} h(\lambda)<$ $\operatorname{deg} f(\lambda)$, then this situation is impossible.

(ii) Let the space $\mathbb{Q}=\left\{g(A) b_{1}, \ldots, g(A) b_{m}\right\} \in \mathbb{P}$ be spanned on the vectors $g(A) b_{1}, \ldots, g(A) b_{m}$; it will be at least 2 -dimensional. It is known that in any cyclic space of dimension $k$ there exist cyclic spaces of all dimensions less than $k$ [15].

Therefore, if $\mathbb{P}_{1} \subset \mathbb{P}$ is a cyclic space of dimension $\operatorname{deg}_{\mathbb{C}} h(\lambda)-1$, then $\mathbb{P}_{1} \cap \mathbb{Q}=$ $g(A) b \neq \emptyset$, where $b \in B(\mathbb{U})$ is a nonzero vector. From here it follows that there exists the polynomial $h_{1}(\lambda)$ such that $h_{1}(A) g(A) b=0$. (Here $\operatorname{deg}_{\mathbb{C}} h_{1}(\lambda) g(\lambda)<$ $\operatorname{deg} f(\lambda)$.) Again we get that the situation (ii) is impossible.

Thus, we have to have $P=\emptyset$ and $\mathbb{X}=\mathbb{X}_{1} \oplus \ldots \oplus \mathbb{X}_{m}$. In addition, $B(\mathbb{U})=$ $\mathbb{X}_{1} \cap B(\mathbb{U}) \oplus \ldots \oplus \mathbb{X}_{m} \cap B(\mathbb{U})=b_{1} \oplus \ldots \oplus b_{m}$. Consequently, there exist bases of the spaces $\mathbb{X}$ and $\mathbb{U}$, in which the matrices $A$ and $B$ can be represent in the following form:

$$
A=\left(\begin{array}{ccc}
A_{1} & & 0 \\
& \ddots & \\
0 & & A_{1}
\end{array}\right), B=\left(\begin{array}{ccc}
B_{1} & & 0 \\
& \ddots & \\
0 & & B_{1}
\end{array}\right),
$$

where $A_{1}=\left.A\right|_{\mathbb{X}_{i}}, B_{1}=b_{i} ; i=1, \ldots, m$. Now the statement of Lemma 3.3 follows from representation (3.3).

Denote by $\mathbb{X} \backslash \mathbb{X}_{1}$ a complement of $\mathbb{X}_{1}$ with respect to whole space $\mathbb{X}$. (Note that $\operatorname{dim}_{\mathbb{C}} \mathbb{X} \backslash \mathbb{X}_{1}=\operatorname{dim}_{\mathbb{C}} \mathbb{X} / \mathbb{X}_{1}$, where $\operatorname{dim}_{\mathbb{C}} \mathbb{X} / \mathbb{X}_{1}$ is a dimension of factor-space $\mathbb{X} / \mathbb{X}_{1}$.)

We choose the bases of spaces $\mathbb{U}$ and $\mathbb{X}$ in accordance with Lemmas 3.1, 3.2, and 3.3. More precisely, the base $B(\mathbb{U})$ is formed by the bases of spaces

$$
B(\mathbb{U}) \cap \mathbb{X}_{1}, B(\mathbb{U}) \cap\left(\mathbb{X}_{2} \backslash \mathbb{X}_{1}\right), \ldots, B(\mathbb{U}) \cap\left(\mathbb{X}_{m} \backslash \mathbb{X}_{m-1}\right),
$$

and the base $\mathbb{X}$ is formed by the bases of spaces

$$
\mathbb{X}_{1}, \mathbb{X}_{2} \backslash \mathbb{X}_{1}, \ldots, \mathbb{X}_{m} \backslash \mathbb{X}_{m-1}
$$


where each of bases $\mathbb{X}_{i+1} \backslash \mathbb{X}_{i}, i=0,1, \ldots, m-1\left(\mathbb{X}_{0}=0\right)$ it is an association of bases of nonintersecting isomorphic cyclic spaces. Then matrices of operators $A$ and $B$ in bases (3.4) and (3.5) can be represented in the following forms:

$$
A=\left(\begin{array}{ccc}
A_{11} & & A_{1 r} \\
& \ddots & \\
0 & & A_{r r}
\end{array}\right), B=\left(\begin{array}{ccc}
B_{11} & & 0 \\
& \ddots & \\
0 & & B_{r r}
\end{array}\right)
$$

where

$$
\begin{gathered}
A_{i i}=\left(\begin{array}{ccc}
A_{i} & & 0 \\
& \ddots & \\
0 & & A_{i}
\end{array}\right), A_{i j}=\left(\begin{array}{ccc}
A_{i j}^{(11)} & \ldots & A_{i j}^{\left(1, m_{j}\right)} \\
\vdots & \ldots & \vdots \\
A_{i j}^{\left(m_{1}, 1\right)} & \ldots & A_{i j}^{\left(m_{i}, m_{j}\right)}
\end{array}\right), \\
A_{i}=\left(\begin{array}{cccc}
0 & & 0 & a_{l_{i}}^{(i)} \\
1 & & 0 & \vdots \\
& \ddots & & \vdots \\
0 & & 1 & a_{1}^{(i)}
\end{array}\right), A_{i j}^{(p q)}=\left(\begin{array}{cccc}
0 & \ldots & 0 & * \\
\vdots & \ldots & \vdots & \vdots \\
0 & \ldots & 0 & *
\end{array}\right) \\
B_{i i}=\left(\begin{array}{ccc}
B_{i} & & \\
& \ddots & \\
0 & & \\
& & \\
& &
\end{array}\right), B_{i}=\left(\begin{array}{c}
1 \\
0 \\
\vdots \\
0
\end{array}\right)
\end{gathered}
$$

Here $m_{i}$ is a multiple of including of block $A_{i}$ in $A_{i i}\left(B_{i}\right.$ in $\left.B_{i i}\right)$; the matrix $A_{i}$ has sizes $l_{i} \times l_{i}$, and matrix $B_{i}$ has sizes $l_{i} \times 1 ; p=1, \ldots, m_{i} ; q=1, \ldots, m_{j}$; $i, j=1, \ldots, r ; i<j$. (In addition, in matrices $A_{i j}^{(p q)}$ only the last column is not equal to zero.)

Let (3.6) be the matrix represent of system $(A, B)$. Denote by $\mathbb{N}=\mathbb{N}_{\mathbb{X}} \times \mathbb{N}_{\mathbb{U}}$ a subset of group $\mathbb{S} L$, which determined as follows:

$$
\begin{aligned}
& \mathbb{N}_{\mathbb{X}} \subset \mathbb{S} L(n, \mathbb{C}), \mathbb{N}_{\mathbb{U}} \subset \mathbb{S} L(m, \mathbb{C}) \\
& \mathbb{N}_{\mathbb{U}}=\left(\begin{array}{ccc}
\mathbb{S} L\left(m_{1}, \mathbb{C}\right) & & \Gamma_{i j} \\
& \ddots & \\
0 & & \mathbb{S} L\left(m_{r}, \mathbb{C}\right)
\end{array}\right), \Gamma_{i j}=\left(\begin{array}{ccc}
\gamma_{i j}^{(11)} & \ldots & \gamma_{i j}^{\left(1, m_{j}\right)} \\
\vdots & \ldots & \vdots \\
\gamma_{i j}^{\left(m_{1}, 1\right)} & \ldots & \gamma_{i j}^{\left(m_{i}, m_{j}\right)}
\end{array}\right) \\
& \mathbb{N}_{\mathbb{X}}=\left(\begin{array}{ccc}
\mathbb{S} L\left(m_{1}, \mathbb{C}\right) \otimes E_{l_{1}} & & L_{i j} \\
& \ddots & \\
0 & & \mathbb{S} L\left(m_{r}, \mathbb{C}\right) \otimes E_{l_{r}}
\end{array}\right)
\end{aligned}
$$


Here $m_{1}+\ldots+m_{r}=m ; L_{i j}=\gamma_{i j}^{(p q)} \cdot\left(B_{i}, \ldots, A_{i}^{l_{j}-1} B_{i}\right) ; \gamma_{i j}^{(p q)}$ are arbitrary parameters; $E_{l_{i}}$ are identity matrices of orders $l_{i} ; m_{1} l_{1}+\ldots+m_{r} l_{r}=n ; p=$ $1, \ldots, m_{i} ; q=1, \ldots, m_{j} ; i<j ;, j=1, \ldots, r$. In this case it is easy to show that the following assertion takes place.

Lemma 3.4. There exist bases of the spaces $\mathbb{X}$ and $\mathbb{U}$, in which $\mathbb{S} t a b_{\mathbb{S} L}(A, B) \subset \mathbb{N}$.

There are a lot of different variants of canonical forms for pair matrices $(A, B)$ (see, for example [20], [33], [45] - [47], [50]). In order to build the stabilizer of system $(A, B)$, we represented own variant (3.6) of such canonical form.

\section{Actions of group $\mathbb{S} L$ on space $\mathbb{S}$}

Denote by $\mathbb{S} / \mathbb{S} L$ a set of orbits all points from the space $\mathbb{S}=\mathbb{C}^{n \times n} \times \mathbb{C}^{n \times m}$ with respect to action (2.3) of group $\mathbb{S} L$.

It is known that for a successful solution of invariant description of system (2.1) it is necessary to supply the space $\mathbb{S}$ (or some suitable subset $\mathbb{W} \subset \mathbb{S}$ ) by a projective variety structure [40], [45] - [47]. The points of this set are called stable. An exact definition of the set of stable points is such.

Definition 4.1. The system $(A, B) \in \mathbb{S}$ is called stable with respect to action (2.3) of group $\mathbb{S} L$, if there exists an open projective $\mathbb{S} L$-invariant set $\mathbb{W} \subset \mathbb{S}$ such that $(A, B) \in \mathbb{W}$ and orbits of all systems from $\mathbb{W}$ are closed in $\mathbb{W}$. A set $\mathbb{F}_{s} \subset \mathbb{S}$ consisting of all stable points is called stable.

Definition 4.2. The point $(A, B)$ is called regular if the dimension of orbit $\mathbb{O}^{\mathbb{S} L}(A, B)$ of this point is maximal in $\mathbb{S}$.

Further, orbits of all points from $\mathbb{F}_{s}$ have the equal (maximal) dimension. Consequently, the stabilizers of these points must have the minimal dimension. Since for regular points is just the equality $\operatorname{dim}_{\mathbb{C}} \mathbb{S} t a b_{\mathbb{S} L}(A, B)=0$, then on the role of stable systems can pretended regular systems only $[32,35,44]$. (We note that the dimension of space of orbits $\mathbb{O}^{\mathbb{G}}(\mathbb{S})$ is given by the formula

$$
\left.\operatorname{dim}_{\mathbb{C}} \mathbb{O}^{\mathbb{S} L}(\mathbb{S})=n^{2}+n m-\operatorname{dim}_{\mathbb{C}} \mathbb{S} L(n, \mathbb{C})-\operatorname{dim}_{\mathbb{C}} \mathbb{S} L(m, \mathbb{C})=m(n-m)+2 .\right)
$$

Theorem 4.1. Let $(A, B)$ be a system of type $(n, m), n>m$. Suppose that at least one of the following conditions is fulfilled:

(i) $m=1$ and $(A, B)$ does not contain nontrivial subsystems;

(ii) $m>1$ and for an arbitrary nontrivial subsystem $\left(\left.A\right|_{\mathbb{X}_{i}},\left.B\right|_{\mathbb{U}_{i}}\right) \in(A, B)$ of type $\left(n_{i}, m_{i}\right), n_{i}>m_{i}$, where $i \in \mathbb{N}$ and $\mathbb{N}$ is a set of indexes, the inequality

$$
\frac{n_{i}}{m_{i}}>\frac{n}{m}
$$

takes place. Then the system $(A, B)$ is regular. 
Corollary. Assume also that the numbers $n$ and $m$ are coprime. Then under the conditions of Theorem 4.1, the system $(A, B) \in \mathbb{F}_{s}$.

Proof. The proof of this theorem is based on Lemmas 3.1-3.4. In essence, it is a modification of the proof proposed in [3].

The following example shows an importance of concept of coprime numbers. Consider the set $\mathbb{S}$ of systems of type $(4,2)$. Let $(A, B)$ be an arbitrary system from this set. Denote by $f(A, B)=\operatorname{det}(B, A B)$ an invariant polynomial of system $(A, B)$. Really, if $(S, T) \in \mathbb{S} L$, then we have

$$
\begin{aligned}
& f\left(S^{-1} A S, S^{-1} B T\right)=\operatorname{det}\left(S^{-1} B T, S^{-1} A B T\right) \\
& =\left((\operatorname{det} T)^{2} / \operatorname{det} S\right) \operatorname{det}(B, A B)=f(A, B) .
\end{aligned}
$$

Consider the following system of type $(4,2)$ :

$$
A=\left(\begin{array}{cccc}
0 & d_{2} & 0 & c_{1} \\
1 & d_{1} & 0 & c_{2} \\
0 & 0 & 0 & d_{2} \\
0 & 0 & 1 & d_{1}
\end{array}\right), \quad B=\left(\begin{array}{ll}
1 & 0 \\
0 & 0 \\
0 & 1 \\
0 & 0
\end{array}\right)
$$

where $c_{1}, c_{2}, d_{1}, d_{2} \in \mathbb{C}$, and $c_{1} \neq 0, c_{2} \neq 0$. In this case, we get

$$
\operatorname{Stab}_{\mathbb{S} L}(A, B)=\{(S, T)\}=\left(\begin{array}{cccc}
1 & 0 & \alpha & 0 \\
0 & 1 & 0 & \alpha \\
0 & 0 & 1 & 0 \\
0 & 0 & 0 & 1
\end{array}\right) \times\left(\begin{array}{cc}
1 & \alpha \\
0 & 1
\end{array}\right), \alpha \neq 0 .
$$

Thus, we have $f(A, B)=1 \neq 0$ and $\operatorname{dim}_{\mathbb{C}} \mathbb{S t a b}_{\mathbb{S} L}(A, B)=1$. Therefore, system (4.3) is not stable.

\section{Invariant description of null-forms for system $(A, B)$}

In Section 2 the existence conditions of null-forms were got in terms of some special subspaces in $\mathbb{X}$ and $\mathbb{U}$. However, for a description of ring of invariants $\mathbb{C}[\mathbb{S}]^{\mathbb{S} L}$, it is necessary to get these conditions in invariant terms of matrix pair $(A, B)$ with respect to action (2.3) of group $\mathbb{S} L$.

Consider the matrix

$$
R(A, B)=\left(B, A B, \ldots, A^{n-1} B\right) \in \mathbb{C}^{n \times n m} .
$$

Then action (2.3) of group $\mathbb{S} L$ on space $\mathbb{C}^{n \times(n+m)}$ induces an action of the same group on space $\mathbb{C}^{n \times n m}$ by the following formula:

$$
R\left(S^{-1} A S, S^{-1} B T\right)=S^{-1} \cdot R(A, B) \cdot\left(\begin{array}{ccc}
T & & 0 \\
& \ddots & \\
0 & & T
\end{array}\right)=S^{-1} \cdot R(A, B) \cdot \bigoplus_{i=1}^{n} T
$$


Many questions of search of invariants for linear control systems may be related to problem of decomposability of polyvectors, which are constructed from vectors of linear space $\mathbb{C}^{n}$. The investigation relationships between polyvectors of $\mathbb{C}^{n}$, alternating multilinear forms on $\mathbb{C}^{n}$, hyperplanes of projective Grassmannians and regular spreads of projective spaces, it were represented in [10]. We use some constructions of this article in our own researches.

Denote by $\mathcal{M}$ a linear space spanned on minors of order $n$ of matrix (5.1). Then in virtue of $(5.2) \mathbb{S} L(\mathcal{M}) \subset \mathcal{M}$. On the space $\mathcal{M}$ group $\mathbb{S} L_{n}$ acts by the multiplication on scalar $\operatorname{det} S=1$, and group $\mathbb{S} L_{m}$ acts in accordance with representation $\bigwedge^{n}\left(\bigoplus_{i=1}^{n} T\right)$. It is known $[35,37]$ that for search of all homogeneous polynomial invariants substantially depending on $B$, it is necessary to find a decomposition of the indicated representation on irreducible components. In obedience to the known result of representation theory of groups an arbitrary irreducible representation of group $\mathbb{S} L_{m}(\mathbb{C})$ is a tensor product of polyvector representations $[10,19]$. Then the decomposition on irreducible components have the form:

$$
\wedge^{n}\left(\bigoplus_{i=1}^{n} T\right)=\underset{\omega=\left(n_{1}, \ldots, n_{d}\right)}{\bigoplus} r_{\omega} \wedge^{n_{1}} T \otimes \wedge^{n_{2}} T \otimes \ldots \otimes \wedge^{n_{d}} T,
$$

where the summation is taken over all multiindexes $\omega=\left(n_{1}, \ldots, n_{d}\right)$ such that $n_{1}+\ldots+n_{d}=n$ and $m \geq n_{1} \geq \ldots \geq n_{d} \geq 1 ; r_{\omega}$ is a multiple of appropriate irreducible representation in representation $\Lambda^{n}\left(\bigoplus_{i=1}^{n} T\right)$.

Below, we construct some examples of invariants for systems of type $(n, m)$.

\subsection{Invariants of systems of type $(4,2)$}

In future by a character $I_{j}(A, B) ; j \in \mathbb{J}$, we will designate an invariant of system $(A, B)$, where $\mathbb{J}$ is a set of indexes.

1) $\omega=\left(n_{1}, n_{2}\right)=(2,2) ; r_{\omega}=1 ; T=\mathbb{S} L(2, \mathbb{C})$, and we consider the representation $T \rightarrow\left(\wedge^{2} T\right) \otimes\left(\wedge^{2} T\right)$. In this case, $I_{j}(A, B)=\operatorname{det}\left(A^{i_{1}} B, A^{i_{2}} B\right)$, where $i_{1}, i_{2}$ are positive integers.

2) $\omega=\left(n_{1}, n_{2}, n_{3}\right)=(2,1,1) ; r_{\omega}=1 ; T=\mathbb{S} L(2, \mathbb{C})$, and we consider the representation $T \rightarrow\left(\wedge^{2} T\right) \otimes T$. In this case

$$
I_{j}(A, B)=\operatorname{det}\left(\begin{array}{cc}
\operatorname{det}\left(B, A^{i_{1}} b_{1}, A^{i_{2}} b_{1}\right) & \operatorname{det}\left(B, A^{i_{1}} b_{1}, A^{i_{2}} b_{2}\right) \\
\operatorname{det}\left(B, A^{i_{1}} b_{2}, A^{i_{2}} b_{1}\right) & \operatorname{det}\left(B, A^{i_{1}} b_{2}, A^{i_{2}} b_{2}\right)
\end{array}\right),
$$

where $\left(b_{1}, b_{2}\right)$ are columns of matrix $B$, and $i_{1}, i_{2}$ are positive integers.

\subsection{Invariants of systems of type $(5,2)$}

1) $\omega=\left(n_{1}, n_{2}, n_{3}\right)=(2,2,1) ; r_{\omega}=1 ; T=\mathbb{S} L(2, \mathbb{C})$, and we consider the representation $T \rightarrow\left(\wedge^{2} T\right) \otimes\left(\wedge^{2} T\right) \otimes T$. In this case

$$
I_{j}(A, B)=\operatorname{det}\left(\begin{array}{cc}
\operatorname{det}\left(B, A^{i_{1}} B, A^{i_{2}} b_{1}\right) & \operatorname{det}\left(B, A^{i_{1}} B, A^{i_{2}} b_{2}\right) \\
\operatorname{det}\left(B, A^{i_{1}} B, A^{i_{3}} b_{1}\right) & \operatorname{det}\left(B, A^{i_{1}} B, A^{i_{3}} b_{2}\right)
\end{array}\right),
$$


where $\left(b_{1}, b_{2}\right)$ are columns of matrix $B$, and $i_{1}, i_{2}, i_{3}$ are positive integers.

\subsection{Invariants of systems of type $(5,3)$}

1) $\omega=\left(n_{1}, n_{2}\right)=(3,2) ; r_{\omega}=1 ; T=\mathbb{S} L(3, \mathbb{C})$, and we consider the representation $T \rightarrow\left(\wedge^{3} T\right) \otimes\left(\wedge^{2} T\right)$. In this case

$$
=\operatorname{det}\left(\begin{array}{ccc}
\operatorname{det}\left(B, A^{i_{1}} b_{1}, A^{i_{1}} b_{2}\right) & \operatorname{det}\left(B, A^{i_{1}} b_{1}, A^{i_{1}} b_{3}\right) & \operatorname{det}\left(B, A^{i_{1}} b_{2}, A^{i_{1}} b_{3}\right) \\
\operatorname{det}\left(B, A^{i_{2}} b_{1}, A^{i_{2}} b_{2}\right) & \operatorname{det}\left(B, A^{i_{2}} b_{1}, A^{i_{2}} b_{3}\right) & \operatorname{det}\left(B, A^{i_{2}} b_{2}, A^{i_{2}} b_{3}\right) \\
\operatorname{det}\left(B, A^{i_{3}} b_{1}, A^{i_{3}} b_{2}\right) & \operatorname{det}\left(B, A^{i_{3}} b_{1}, A^{i_{3}} b_{3}\right) & \operatorname{det}\left(B, A^{i_{3}} b_{2}, A^{i_{3}} b_{3}\right)
\end{array}\right),
$$

where $\left(b_{1}, b_{2}, b_{3}\right)$ are columns of matrix $B$, and $i_{1}, i_{2}, i_{3}$ are positive integers.

2) $\omega=\left(n_{1}, n_{2}, n_{3}\right)=(3,1,1) ; r_{\omega}=1 ; T=\mathbb{S} L(3, \mathbb{C})$, and we consider the representation $T \rightarrow\left(\wedge^{3} T\right) \otimes T \otimes T$. In this case

$$
=\operatorname{det}\left(\begin{array}{ccc}
\operatorname{det}\left(B, A^{i_{1}} b_{1}, A^{i_{2}} b_{1}\right) & \operatorname{det}\left(B, A^{i_{1}} b_{1}, A^{i_{2}} b_{2}\right) & \operatorname{det}\left(B, A^{i_{1}} b_{1}, A^{i_{2}} b_{3}\right) \\
\operatorname{det}\left(B, A^{i_{1}} b_{2}, A^{i_{2}} b_{1}\right) & \operatorname{det}\left(B, A^{i_{1}} b_{2}, A^{i_{2}} b_{2}\right) & \operatorname{det}\left(B, A^{i_{1}} b_{2}, A^{i_{2}} b_{3}\right) \\
\operatorname{det}\left(B, A^{i_{1}} b_{3}, A^{i_{3}} b_{1}\right) & \operatorname{det}\left(B, A^{i_{1}} b_{3}, A^{i_{3}} b_{2}\right) & \operatorname{det}\left(B, A^{i_{1}} b_{3}, A^{i_{3}} b_{3}\right)
\end{array}\right),
$$

where $\left(b_{1}, b_{2}, b_{3}\right)$ are columns of matrix $B$, and $i_{1}, i_{2}, i_{3}$ are positive integers.

Now we can begin to construct null-forms for systems of type $(n, m)$. Denote by $a_{i}(A), i=1, \ldots, n$, the coefficients of characteristic polynomial of matrix $A$. In addition, by the symbol $\mathbb{W}^{\circ}(n, m)$ we will denote a variety of all null-forms of space $\mathbb{S}$ with respect to action (2.3) of group $\mathbb{S} L$.

\subsection{Null-forms of systems of type $(m+1, m), m>1$}

Construct the following matrix:

$$
R(A, B)=\left(\begin{array}{ccc}
\operatorname{det}\left(B, A b_{1}\right), & \cdots & , \operatorname{det}\left(B, A b_{m}\right) \\
\vdots & \cdots & \vdots \\
\operatorname{det}\left(B, A^{m} b_{1}\right), & \cdots & , \operatorname{det}\left(B, A^{m} b_{m}\right)
\end{array}\right) \in \mathbb{C}^{m \times m}
$$

Consider the invariant $I_{1}(A, B)=\operatorname{det} R(A, B)$ of matrix (5.3).

Theorem 5.1. Let $n=m+1, m \geq 1$. Then

$\mathbb{W}^{\circ}(m+1, m)=\left\{(A, B) \in \mathbb{C}^{n \times(n+m)} \mid a_{1}(A)=\ldots=a_{n}(A)=0\right.$ and $\left.I_{1}(A, B)=0\right\}$.

Proof. Assume that equality $I_{1}(A, B)=0$ takes place. Then form (5.3) of matrix $R(A, B)$ allows to assert that columns of this matrix are linearly dependent. In other words, there exist numbers $\alpha_{1}, \ldots, \alpha_{m} \in \mathbb{C}$ not all equal to zero such that

$$
\left(\begin{array}{c}
\operatorname{det}\left(B, A\left(\alpha_{1} b_{1}+\ldots+\alpha_{m} b_{m}\right)\right) \\
\vdots \\
\operatorname{det}\left(B, A^{m}\left(\alpha_{1} b_{1}+\ldots+\alpha_{m} b_{m}\right)\right)
\end{array}\right)=0 .
$$


It is possible to consider that $\operatorname{rank} B=m$. (Otherwise the system $(A, B)$ is the null-form.) Then from (5.4) it follows that $A^{i}\left(\alpha_{1} b_{1}+\ldots+\alpha_{m} b_{m}\right) \in B(\mathbb{U}), i=$ $1, \ldots, n-1$, and, consequently, the space $B(\mathbb{U})$ contains the invariant subspace $\mathbb{V}$ spanned on the vectors

$$
\left(\alpha_{1} b_{1}+\ldots+\alpha_{m} b_{m}\right), A\left(\alpha_{1} b_{1}+\ldots+\alpha_{m} b_{m}\right), \ldots, A^{n-1}\left(\alpha_{1} b_{1}+\ldots+\alpha_{m} b_{m}\right) .
$$

It is known that an arbitrary invariant space of matrix $A$ contains an eigenvector of this matrix [15]. Thus, there is a nonzero vector $b \in \mathbb{V} \subset B(\mathbb{U})$ such that $A b=\lambda b$, where $\lambda$ is an eigenvalue of matrix $A$. It means that in the system $(A, B)$ there exists the subsystem of type $(1,1)$. In addition, if $a_{1}(A)=\ldots=a_{n}(A)=0$, then in accord to Theorem 2.2 the system $(A, B)$ is the null-form.

\subsection{Null-forms of systems of type $(2 m, m), m>1$}

Denote by $\alpha_{1}, \ldots, \alpha_{n}$ eigenvalues of matrix $A$ and let

$$
\operatorname{disc}(A)=\left(\alpha_{2}-\alpha_{1}\right)^{2}\left(\alpha_{3}-\alpha_{1}\right)^{2} \cdot \ldots \cdot\left(\alpha_{n}-\alpha_{1}\right)^{2} \cdot \ldots \cdot\left(\alpha_{n}-\alpha_{n-1}\right)^{2}
$$

be a discriminant of this matrix.

Let

$$
\operatorname{Bin}(\eta, \xi)=\left(\begin{array}{c}
\eta \\
\xi
\end{array}\right)=\frac{\eta !}{\xi !(\eta-\xi) !} \equiv \frac{\eta \cdot(\eta-1) \cdot \ldots \cdot(\eta-\xi+1)}{1 \cdot \ldots \cdot \xi}
$$

be a binomial coefficient. Here $\eta, \xi$ are positive integers, $0 \leq \xi \leq \eta ; 0 !=1 !=1$.

Introduce the invariants

$$
I_{j}(A, B)=\operatorname{det}\left(B, A^{j-1} B\right),
$$

where $j=1, \ldots, \operatorname{Bin}(2 m, m) / 2$. (Note that $I_{1}(A, B) \equiv 0$.)

Theorem 5.2. Let $n=2 m, m \geq 1$. Then

$\mathbb{W}^{\circ}(2 m, m)=\left\{(A, B) \in \mathbb{C}^{n \times(n+m)} \mid a_{1}(A)=\ldots=a_{n}(A)=0\right.$ and $\left.I_{j}(A, B)=0\right\}$, where $I_{j}(A, B)$ are invariants (5.5).

Proof. Denote by $\mathbb{M}$ an open set in $\mathbb{C}^{n \times(n+m)}$ is given by the condition:

$$
\mathbb{M}=\left\{(A, B) \in \mathbb{C}^{n \times(n+m)} \mid \operatorname{disc}(A) \neq 0\right\} .
$$

Let $(A, B) \in \mathbb{M}$ be an arbitrary system. Then it is possible to consider that in a suitable base of space $\mathbb{X}$ the matrix $A=\operatorname{diag}\left(() \alpha_{1}, \ldots, \alpha_{2 m}\right)$.

Denote by $\Delta_{j_{1} \ldots j_{m}}$ a minor located in the rows $1 \leq j_{1}<\ldots<j_{m} \leq 2 m$ of matrix $B$.

Let $\gamma_{1}=\alpha_{1} \cdot \ldots \cdot \alpha_{m}, \delta_{1}=\alpha_{m+1} \cdot \ldots \cdot \alpha_{2 m}, \ldots, \gamma_{k}=\alpha_{i_{i}} \cdot \ldots \cdot \alpha_{i_{m}}, \delta_{k}=\alpha_{j_{1}} \cdot \ldots \cdot \alpha_{j_{m}}$, where $i_{1}, \ldots, i_{m}, j_{1}, \ldots, j_{m} \in\{1,2, \ldots, 2 m\} ; 1 \leq i_{1}<\ldots<i_{m} \leq 2 m, 1 \leq j_{1}<\ldots<$ $j_{m} \leq 2 m$, and $\left(i_{1}, \ldots, i_{m}\right) \cap\left(j_{1}, \ldots, j_{m}\right)=\emptyset ; k=\operatorname{Bin}(2 m, m) / 2$. 
Construct the matrix

$$
Q\left(\alpha_{1}, \ldots, \alpha_{2 m}\right)=\left(\begin{array}{ccccc}
2 & \cdots & 2 & \cdots & 2 \\
\gamma_{1}+\delta_{1} & \cdots & \gamma_{i_{1}}+\delta_{j_{1}} & \cdots & \gamma_{k}+\delta_{k} \\
\gamma_{1}^{2}+\delta_{1}^{2} & \cdots & \gamma_{i_{1}}^{2}+\delta_{j_{1}}^{2} & \cdots & \gamma_{k}^{2}+\delta_{k}^{2} \\
\vdots & \cdots & \vdots & \cdots & \vdots \\
\gamma_{1}^{k-1}+\delta_{1}^{k-1} & \cdots & \gamma_{i_{1}}^{k-1}+\delta_{j_{1}}^{k-1} & \cdots & \gamma_{k}^{k-1}+\delta_{k}^{k-1}
\end{array}\right) \in \mathbb{C}^{k \times k}
$$

Taking into account the matrix $Q\left(\alpha_{1}, \ldots, \alpha_{2 m}\right)$, the conditions $I_{j}(A, B)=0$ may be rewritten as

$$
\left(\begin{array}{c}
I_{1} \\
\vdots \\
I_{k}
\end{array}\right)=Q\left(\alpha_{1}, \ldots, \alpha_{2 m}\right) \cdot\left(\begin{array}{c}
\Delta_{1 \ldots m} \cdot \Delta_{m+1 \ldots 2 m} \\
\vdots \\
\Delta_{i_{1} \ldots i_{m}} \cdot \Delta_{j_{1} \ldots j_{m}}
\end{array}\right) .
$$

Assume that $\operatorname{det} Q\left(\alpha_{1}, \ldots, \alpha_{2 m}\right) \neq 0$. It is clear that if $I_{1}=\ldots=I_{k}=0$, then for all indicated above possible collections of indexes $i_{1} \ldots i_{m}, j_{1} \ldots j_{m}$ such that $\left\{i_{1} \ldots i_{m}\right\} \cap\left\{j_{1} \ldots j_{m}\right\}=0$, system (5.6) has the trivial solutions only: $\Delta_{i_{1} \ldots i_{m}}$. $\Delta_{j_{1} \ldots j_{m}}=0$.

Further, invariant (5.5) can be written as:

$$
\operatorname{det}\left(B, A^{j-1} B\right)=\sum_{i=1}^{k}(-1)^{i} f_{i j}\left(\alpha_{1}, \ldots, \alpha_{2 m}\right) \Delta_{i_{1} \ldots i_{k}} \cdot \Delta_{j_{1} \ldots j_{k}},
$$

where $\left\{i_{1}, \ldots, i_{m}, j_{1} \ldots j_{m}\right\}=\{1, \ldots, 2 m\}, 1 \leq i_{1}<\ldots<i_{m} \leq 2 m ; 1 \leq j_{1}<\ldots<$ $j_{m} \leq 2 m$.

From (5.7) it follows that equalities $\Delta_{i_{1} \ldots i_{m}} \cdot \Delta_{j_{1} \ldots j_{m}}=0$ take place if and only if the matrix $(B, B)$ has the following form:

$$
(B, B)=\left(\begin{array}{cccccc||cccccc}
* & \cdots & * & * & \cdots & * \\
\vdots & \cdots & \vdots & \vdots & \cdots & \vdots \\
* & \cdots & * & * & \cdots & * \\
0 & \cdots & 0 & * & \cdots & * & \cdots & \cdots & * & * & \cdots & * \\
\vdots & \cdots & \vdots & \vdots & \cdots & \vdots \\
* & \cdots & * & * & \cdots & * \\
0 & N & \vdots & \vdots & \cdots & \vdots & 0 & * & \cdots & * \\
0 & \cdots & 0 & * & \cdots & * & \cdots & N & \vdots & \vdots & \cdots & \vdots \\
0 & \cdots & 0 & * & \cdots & *
\end{array}\right) \in \mathbb{C}^{2 m \times 2 m}
$$

where $N \in \mathbb{C}^{(2 m-2 l+1) \times l}$ is the zero submatrix of matrix $B$ and the symbol $*$ designates a nonzero element of matrix $B ; l=m-1, m-2, \ldots, 1$.

It means that the space $B(\mathbb{U})$ intersects with the invariant subspace $\mathbb{V} \subset$ $\mathbb{C}^{2 m}(A \mathbb{V} \subset \mathbb{V})$ such that $\operatorname{dim}_{\mathbb{C}} \mathbb{V} \leq 2 l-1$ and $\operatorname{dim}_{\mathbb{C}} B(\mathbb{U}) \cap \mathbb{V}=l$, where $l=$ $m-1, \ldots, 1$. Consequently, the system $(A, B)$ of type $(2 m, m)$ contains a subsystem of type $(2 l-1, l) ; l \in\{m-1, \ldots, 1\}$. Thus, we have $(2 l-1) / l<2 m / m=2$ for $l>0$. 
Denote by $\mathbb{K}$ an open set in $\mathbb{C}^{n \times(n+m)}$ is given by the following condition:

$$
\mathbb{K}=\left\{(A, B) \in \mathbb{C}^{n \times(n+m)} \mid \operatorname{det} Q\left(\alpha_{1}, \ldots, \alpha_{2 m}\right) \cdot \operatorname{disc}(A) \neq 0\right\} .
$$

It is known [44] that if the regular function $I_{j}(A, B)=0$ on the open set $\mathbb{K} \subset \mathbb{C}^{n \times(n+m)}$, then this function is equal to zero everywhere on $\mathbb{C}^{n \times(n+m)}$. Now we add conditions $I_{j}(A, B)=0, j=2, \ldots, k$, by conditions $a_{i}(A)=0, i=1, \ldots, 2 m$. Then according to Theorem 2.2, we obtain that $(A, B)$ is the null-form.

\subsection{Null-forms of systems of type $(p m, m), p>2, m>1$}

In this subsection the results of previous subsection will be generalized.

Introduce the invariants

$$
I_{j}(A, B)=\operatorname{det}\left(B, A B, \ldots, A^{p-2} B, A^{j} B\right),
$$

where $j=p-1, \ldots, \operatorname{Bin}(p m, m)$.

Theorem 5.3. Let $n=p m, p>2, m \geq 1$. Then

$\mathbb{W}^{\circ}(p m, m)=\left\{(A, B) \in \mathbb{C}^{n \times(n+m)} \mid a_{1}(A)=\ldots=a_{n}(A)=0\right.$ and $\left.I_{j}(A, B)=0\right\}$, where $I_{j}(A, B)$ are invariants (5.8).

Proof. A proof of this theorem almost word for a word repeats the proof of Theorem 5.2. It is necessary only to specify some details.

Let $\operatorname{det} Q\left(\alpha_{1}, \ldots, \alpha_{p m}\right) \neq 0$. Then equations (5.6), (5.7), and the matrix $(B, B)$ are replaced accordingly by equations

$$
\begin{gathered}
\left(\begin{array}{c}
I_{1} \\
\vdots \\
I_{r}
\end{array}\right)=Q\left(\alpha_{1}, \ldots, \alpha_{p m}\right) \cdot\left(\begin{array}{c}
\Delta_{1 \ldots m} \cdot \ldots \cdot \Delta_{(p-1) m+1 \ldots p m} \\
\vdots \\
\Delta_{i_{1} \ldots i_{m}} \cdot \ldots \cdot \Delta_{j_{1} \ldots j_{m}}
\end{array}\right), \\
\operatorname{det}\left(B, A B, \ldots, A^{p-1} B, A^{j-1} B\right)=\sum_{i=1}^{r}(-1)^{i} f_{i j}\left(\alpha_{1}, \ldots, \alpha_{p m}\right) \Delta_{i_{1} \ldots i_{1}} \cdot \ldots \cdot \Delta_{j_{1} \ldots j_{k}},
\end{gathered}
$$

where $r=\operatorname{Bin}(p m, m) ;\left\{i_{1}, \ldots, i_{m}, \ldots, j_{1}, \ldots, j_{m}\right\}=\{1, \ldots, p m\} ; 1 \leq i_{1}<\ldots<$ $i_{m} \leq p m ; \ldots ; 1 \leq j_{1}<\ldots<j_{m} \leq p m$, and the matrix

$$
\underbrace{(B, \ldots, B)}_{p m}=\left(\begin{array}{cccccc}
* & \cdots & * & * & \cdots & * \\
\vdots & \cdots & \vdots & \vdots & \cdots & \vdots \\
* & \cdots & * & * & \cdots & * \\
0 & \cdots & 0 & * & \cdots & * \\
\vdots & N & \vdots & \vdots & \cdots & \vdots \\
0 & \cdots & 0 & * & \cdots & *
\end{array}|\cdots| \begin{array}{cccccc}
* & \cdots & * & * & \cdots & * \\
\vdots & \cdots & \vdots & \vdots & \cdots & \vdots \\
* & \cdots & * & * & \cdots & * \\
0 & \cdots & 0 & * & \cdots & * \\
\vdots & N & \vdots & \vdots & \cdots & \vdots \\
0 & \cdots & 0 & * & \cdots & *
\end{array}\right) \in \mathbb{C}^{p m \times p m} .
$$


In order that $I_{1}=\ldots=I_{r}=0$, it is necessary that $\Delta_{i_{1} \ldots i_{m}} \cdot \ldots \cdot \Delta_{j_{1} \ldots j_{m}}=0$. Then from $(5.9),(5.10)$, and the representation of matrix $(B, \ldots, B)$ it follows that the space $B(\mathbb{U})$ intersects with the invariant subspace $\mathbb{V} \subset \mathbb{C}^{p m}(A \mathbb{V} \subset \mathbb{V})$ such that $\operatorname{dim}_{\mathbb{C}} \mathbb{V} \leq p l-1$ and $\operatorname{dim}_{\mathbb{C}} B(\mathbb{U}) \cap \mathbb{V}=l$, where $l=m-1, \ldots, 1$. Consequently, the system $(A, B)$ of type $(p m, m)$ contains a subsystem of type $(p l-1, l) ; l \in\{m-1, \ldots, 1\}$. Thus, we have $(p l-1) / l<p m / m=p$ for $l>0$.

5.7. Null-forms of systems of type $(p m+1, m), p \geq 2, m>1$

Let $B=\left(b_{1}, \ldots, b_{m}\right)$. Introduce the matrix $R_{1}(A, B) \in \mathbb{C}^{(p m-m+1) \times m}$, where

$$
=\left(\begin{array}{ccc}
\operatorname{det}\left(B, \ldots, A^{p-1} B, A^{p} b_{1}\right) & \cdots & \operatorname{det}\left(B, \ldots, A^{p-1} B, A^{p} b_{m}\right) \\
\vdots & \cdots & \vdots \\
\operatorname{det}\left(B, \ldots, A^{p-1} B, A^{p m} b_{1}\right) & \cdots & \operatorname{det}\left(B, \ldots, A^{p-1} B, A^{p m} b_{m}\right)
\end{array}\right) .
$$

Introduce the invariants $I_{1 j}(A, B)$, which are all minors of degree $m$ of matrix $R_{1}(A, B) ; j=1, \ldots, \operatorname{Bin}(p m, m)$.

Theorem 5.4. Let $n=p m+1, p \geq 2, m>1$. Then

$$
\begin{gathered}
\mathbb{W}^{\circ}(p m+1, m)=\left\{(A, B) \in \mathbb{C}^{n \times(n+m)}\right. \\
\left.\mid a_{1}(A)=\ldots=a_{n}(A)=0 ; I_{1 j}(A, B)=0, j=1, \ldots, \operatorname{Bin}(p m, m)\right\} .
\end{gathered}
$$

Proof. Assume that equalities $I_{1 j}(A, B)=0, j=1, \ldots, B i n(p m, m)$, take place. Then from (5.11) it follows that columns of matrix $R_{1}(A, B)$ are linearly dependent. Consequently, there exist numbers $\alpha_{1}, \ldots, \alpha_{m} \in \mathbb{C}$ not all equal to zero such that

$$
\left(\begin{array}{c}
\operatorname{det}\left(B, A B, \ldots, A^{p-1} B, A^{p}\left(\alpha_{1} b_{1}+\ldots+\alpha_{m} b_{m}\right)\right) \\
\vdots \\
\operatorname{det}\left(B, A B, \ldots, A^{p-1} B, A^{p m}\left(\alpha_{1} b_{1}+\ldots+\alpha_{m} b_{m}\right)\right)
\end{array}\right)=0 .
$$

Equalities (5.12) can be rewritten as $\wedge^{p m+1}\left(B, A B, \ldots, A^{p-1} B, A^{j} b\right)=0$, where $b=\alpha_{1} b_{1}+\ldots+\alpha_{m} b_{m}=\left(\Delta_{1}, \ldots, \Delta_{p m+1}\right)^{T}, \Delta_{1}, \ldots, \Delta_{p m+1}$ are coordinates of vector $b, j=p, \ldots, p m$.

Thus, we have

$$
I_{1 j}(A, B)=\sum_{i=1}^{k}(-1)^{i} f_{i j}\left(\alpha_{1}, \ldots, \alpha_{p m+1}\right) \Delta_{i_{1} \ldots i_{m}} \cdot \ldots \cdot \Delta_{j_{1} \ldots j_{m}} \cdot \ldots \cdot \Delta_{l_{1} \ldots l_{m}} \cdot \Delta_{i}
$$

where $\left\{i_{1}, \ldots, i_{2}, \ldots, j_{1}, \ldots, j_{m}, \ldots, l_{1}, \ldots, l_{m}, i\right\}=\{1, \ldots, p m+1\} ; k=\operatorname{Bin}(p m, m)$.

From (5.13) it follows that equalities $\Delta_{i_{1} \ldots i_{m}} \cdot \ldots \cdot \Delta_{j_{1} \ldots j_{m}} \cdot \ldots \cdot \Delta_{l_{1} \ldots l_{m}} \cdot \Delta_{i}=0$ take place if and only if the matrix $(B, \ldots, B, b)$ has the form

$$
\underbrace{(B, \ldots, B, b)}_{p m+1}
$$




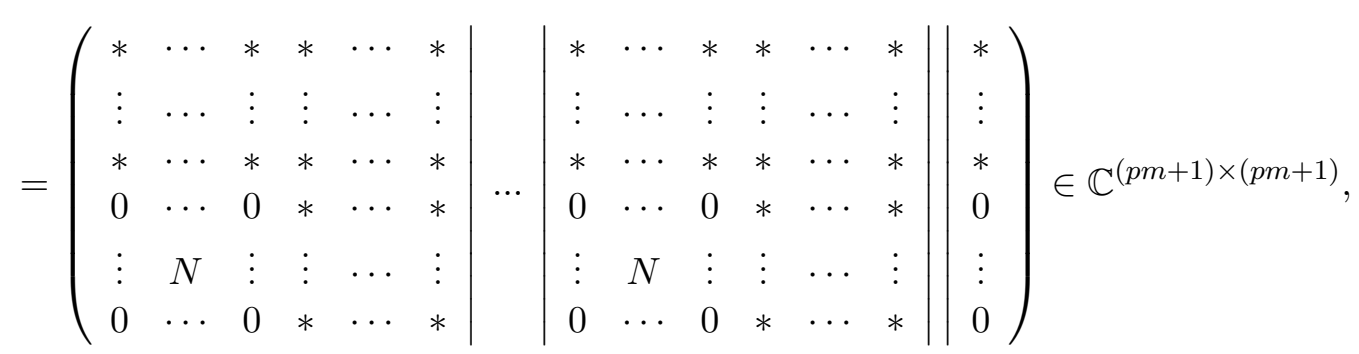

where $N \in \mathbb{C}^{(p m-p l+1) \times l}$ is the zero submatrix of matrix $B$ and the symbol $*$ is a nonzero element of matrix $B ; l=m-1, m-2, \ldots, 1$. In addition, the column $b$ has $p m-p l+1$ zero coordinates.

In order that $I_{11}=\ldots=I_{1 r}=0$, it is necessary that $\Delta_{i_{1} \ldots i_{m}} \cdot \ldots \cdot \Delta_{j_{1} \ldots j_{m}} \cdot \ldots \cdot$ $\Delta_{l_{1} \ldots l_{m}} \cdot \Delta_{i}=0$. Then from the representation of matrix $(B, \ldots, B, b)$ it follows that the space $B(\mathbb{U})$ intersects with the invariant subspace $\mathbb{V} \subset \mathbb{C}^{p m+1}(A \mathbb{V} \subset \mathbb{V})$ such that $\operatorname{dim}_{\mathbb{C}} \mathbb{V} \leq p l$ and $\operatorname{dim}_{\mathbb{C}} B(\mathbb{U}) \cap \mathbb{V}=l$, where $l=m-1, \ldots, 1$. Consequently, the system $(A, B)$ of type $(p m+1, m)$ contains a subsystem of type $(p l, l) ; l \in$ $\{m-1, \ldots, 1\}$. Thus, we have $p l / l=p<(p m+1) / m$ for $l>0$. Then according to Theorem 2.2, we obtain that $(A, B)$ is the null-form.

5.8. Null-forms of systems of type $(p m-1, m), p \geq 2, m>1$

Let $B=\left(b_{1}, \ldots, b_{m}\right)$. Introduce the matrix $R_{m-1}(A, B) \in \mathbb{C}^{(p m-m+1) \times m}$, where

$$
\begin{gathered}
R_{m-1}(A, B) \\
=\left(\begin{array}{ccc}
\operatorname{det}\left(B, \ldots, A^{p}\left(b_{1}, \ldots, b_{m-1}\right)\right) & \cdots & \operatorname{det}\left(B, \ldots, A^{p}\left(b_{2}, \ldots, b_{m}\right)\right) \\
\vdots & \ldots & \vdots \\
\operatorname{det}\left(B, \ldots, A^{p m}\left(b_{1}, \ldots, b_{m-1}\right)\right) & \cdots & \operatorname{det}\left(B, \ldots, A^{p m}\left(b_{2}, \ldots, b_{m}\right)\right)
\end{array}\right) .
\end{gathered}
$$

Introduce the invariants $I_{m-1, j}(A, B) ; j=1, \ldots, \operatorname{Bin}(p m, m)$, which are all minors of degree $m$ of matrix $R_{m-1}(A, B)$.

Theorem 5.5. Let $n=p m-1, p \geq 2, m>1$. Then

$$
\begin{gathered}
\mathbb{W}^{\circ}(p m-1, m)=\left\{(A, B) \in \mathbb{C}^{n \times(n+m)}\right. \\
\left.\mid a_{1}(A)=\ldots=a_{n}(A)=0 ; I_{m-1, j}(A, B)=0, j=1, \ldots, \operatorname{Bin}(p m, m)\right\} .
\end{gathered}
$$

Proof. Assume that equalities $I_{m-1, j}(A, B)=0, j=1, \ldots, \operatorname{Bin}(p m, m)$, take place. Then from (5.14) it follows that columns of matrix $R_{m-1}(A, B)$ are linearly dependent. Consequently, there exist numbers $\alpha_{1}, \ldots, \alpha_{m} \in \mathbb{C}$ not all equal to zero such that

$\left(\begin{array}{c}\operatorname{det}\left(B, A B, \ldots, A^{p-1} B, \alpha_{1} A^{p}\left(b_{1}, \ldots, b_{m-1}\right)+\ldots+\alpha_{m} A^{p}\left(b_{m-1}, \ldots, b_{m}\right)\right) \\ \vdots \\ \operatorname{det}\left(B, A B, \ldots, A^{p-1} B, \alpha_{1} A^{p m}\left(b_{1}, \ldots, b_{m-1}\right)+\ldots+\alpha_{m} A^{p}\left(b_{m-1}, \ldots, b_{m}\right)\right)\end{array}\right)=0$. 
According to the known result of external algebra $[10,19]$ any $(m-1)$-polyvector built from $m-1$ vectors of $m$-dimensional space $B(\mathbb{U})$ is simple. In considered case it means that there exist numbers $\alpha_{1}, \ldots, \alpha_{m} \in \mathbb{C}$ not all equal to zero such that

$$
\wedge^{m-1}\left(\alpha_{1}\left(b_{1}, \ldots, b_{m-1}\right)+\ldots+\alpha_{m}\left(b_{m-1}, \ldots, b_{m}\right)\right)=\wedge^{m-1}\left(q_{1}, \ldots, q_{m-1}\right),
$$

where $q_{i} \in B(\mathbb{U}), i=1, \ldots, m-1$.

Without loss of generality, it is possible to consider that $b_{1}=q_{1}, \ldots, b_{m-1}=$ $q_{m-1}$. Then equalities (5.15) can be rewritten as

$$
\wedge^{p m-1}\left(B, A B, \ldots, A^{p-1} B, A^{j}\left(b_{1}, \ldots, b_{m-1}\right)\right)=0,
$$

where $j=p, \ldots, p m$.

Thus, we have

$$
I_{m-1, j}(A, B)=\sum_{i=1}^{k}(-1)^{i} f_{i j}\left(\alpha_{1}, \ldots, \alpha_{p m-1}\right) \Delta_{i_{1} \ldots i_{m}} \cdot \ldots \Delta_{j_{1} \ldots j_{m}} \cdot \Delta_{l_{1} \ldots l_{m-1}}
$$

where $\left\{i_{1}, \ldots, i_{m}, \ldots, j_{1}, \ldots, j_{m}, l_{1}, \ldots ., l_{m-1}\right\}=\{1, \ldots, p m-1\}, k=\operatorname{Bin}(p m, m)$, and $\Delta_{l_{1} \ldots l_{m-1}}$ are minors of matrix $\left(b_{1}, \ldots, b_{m-1}\right)$ of order $m-1$.

From (5.16) it follows that equalities $\Delta_{i_{1} \ldots i_{m}} \cdot \ldots \cdot \Delta_{j_{1} \ldots j_{m}} \cdot \Delta_{l_{1} \ldots l_{m-1}}=0$ take place if and only if the matrix $\left(B, \ldots, B, b_{1}, \ldots, b_{m-1}\right)$ has the form:

$$
\begin{aligned}
& \underbrace{\left(B, \ldots, B, b_{1}, \ldots, b_{m-1}\right)}_{p m-1}
\end{aligned}
$$

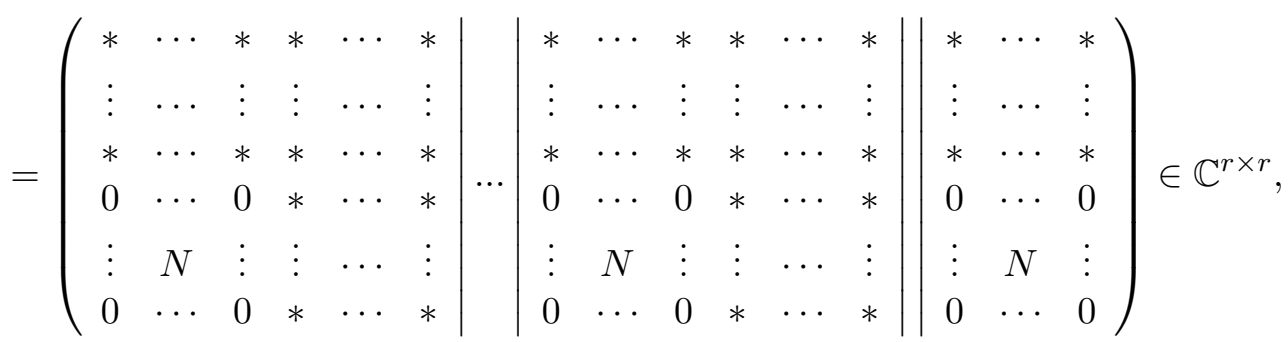

where $r=p m-1, N \in \mathbb{C}^{(p m-p l+1) \times l}$ is the zero submatrix of matrix $B$, and the symbol $*$ is a nonzero element of matrix $B ; l=m-1, m-2, \ldots, 1$.

In order that $I_{m-1,1}=\ldots=I_{m-1, k}=0$, it is necessary that $\Delta_{i_{1} \ldots i_{m}} \cdot \ldots \cdot \Delta_{j_{1} \ldots j_{m}}$. $\Delta_{l_{1} \ldots l_{m-1}}=0$. Then from the representation of matrix $\left(B, \ldots, B, b_{1}, \ldots, b_{m-1}\right)$ it follows that the space $B(\mathbb{U})$ intersects with the invariant subspace $\mathbb{V} \subset \mathbb{C}^{p m-1}$ $(A \mathbb{V} \subset \mathbb{V})$ such that $\operatorname{dim}_{\mathbb{C}} \mathbb{V} \leq p l$ and $\operatorname{dim}_{\mathbb{C}} B(\mathbb{U}) \cap \mathbb{V}=l$, where $l=m-1, \ldots, 1$. Consequently, the system $(A, B)$ of type $(p m-1, m)$ contains a subsystem of type $(p l-1, l) ; l \in\{m-1, \ldots, 1\}$. Thus, we have $(p l-1) / l<(p m-1) / m$ for $l>0$. Then according to Theorem 2.1, we obtain that $(A, B)$ is the null-form. 


\section{Stability criterions for system $(A, B)$}

Now we explain an importance of stability concept. Denote by $\mathbb{C}[A, B]^{\mathbb{S} L}$ the ring of all invariants of space $\mathbb{C}^{n \times(n+m)}$ with respect to action (2.3) of group $\mathbb{S} L$. Assume that

$$
a_{1}=a_{1}(A), \ldots, a_{n}=a_{n}(A), I_{1}=I_{1}(A, B), \ldots, I_{r}=I_{r}(A, B)
$$

is a set of homogeneous polynomial generators of ring $\mathbb{C}[A, B]^{\mathbb{S} L}$. (Since the group $\mathbb{S} L$ is reductive, then the set of generators is finite [35].) Thus, it is possible to write $\mathbb{C}[A, B]^{\mathbb{S L}}=\mathbb{C}\left[a_{1}, \ldots, a_{n}, I_{1}, \ldots, I_{r}\right]$.

Consider two systems $\left(A_{1}, B_{1}\right),\left(A_{2}, B_{2}\right) \in \mathbb{C}^{n \times(n+m)}$, which are determined by the same set of invariants: $a_{1}, \ldots, a_{n}, I_{1}, \ldots, I_{r}$. (In other words we have to have: $a_{1}\left(A_{1}\right)=a_{1}\left(A_{2}\right), \ldots, a_{n}\left(A_{1}\right)=a_{n}\left(A_{2}\right), I_{1}\left(A_{1}, B_{1}\right)=I_{1}\left(A_{2}, B_{2}\right), \ldots, I_{r}\left(A_{1}, B_{1}\right)=$ $I_{r}\left(A_{2}, B_{2}\right)$.) Are there matrices $S \in \mathbb{G} L(n, \mathbb{C})$ and $T \in \mathbb{G} L(m, \mathbb{C})$ such that $A_{2}=S^{-1} A_{1} S$ and $B_{2}=S^{-1} B_{1} T$ ? The example of system (4.3) shows that the answer on this question is negative.

In $[35,45]$ it is shown that the set of invariants $a_{1}(A), \ldots, a_{n}(A), I_{1}(A, B), \ldots$, $I_{r}(A, B)$ determines a unique $\mathbb{S} L$-orbit (or $\mathbb{G} L$-orbit) of system $(A, B)$ if and only if $(A, B) \in \mathbb{F}_{s}$. This circumstance explains the importance of stability concept.

The following theorem specifies Theorem 4.1.

Assume that $n=(p-1) m+s, 1<s<m$, where $s$ is a natural number. Consider in the representation $\bigwedge^{n}\left(\bigoplus_{i=1}^{n} T\right)$ simplest components of kind $\wedge^{m} T^{\otimes(p-1)} \otimes$ $\wedge^{s} T=\operatorname{det}(T)^{p-1} \otimes \wedge^{s} T$.

Introduce the invariant $f_{s}(A, B)=\operatorname{det}\left(\mathbf{z}_{1}, \ldots, \mathbf{z}_{l}\right), l=\operatorname{Bin}(m, s)$, where all vectors $\mathbf{z}_{i}$ belong to different submodules of kind $\wedge^{m} T^{\otimes(p-1)} \otimes \wedge^{s} T=\operatorname{det}(T)^{p-1} \otimes$ $\wedge^{s} T$. For the systems $(A, B)$ of type $(n, m)$ indicated invariant is $f_{s}(A, B)=$ $\operatorname{det} G(A, B)$, and elements of square matrix $G(A, B)$ of order $l=\operatorname{Bin}(m, s)$ are:

$$
g_{i j}=\operatorname{det}\left(B, A B, \ldots, A^{p-1} B, A^{j}\left(b_{i_{1}}, \ldots, b_{i_{s}}\right)\right), j \geq p .
$$

Theorem 6.1. Let number $n$ and $m$ be coprime numbers and $f_{s}(A, B)=\operatorname{det}\left(\mathbf{z}_{1}\right.$, $\left.\ldots, \mathbf{z}_{l}\right)$ be an invariant substantially depending on $B$. We will assume that vectors $\mathbf{z}_{1}, \ldots, \mathbf{z}_{l}$ belong to different components of representation $\bigwedge^{n}\left(\bigoplus_{i=1}^{n} T\right)$, which are isomorphic to $(\operatorname{det} T)^{p-1} \otimes \wedge^{s} T$. If $f_{s}(A, B) \neq 0$, then system $(A, B)$ is stable.

Proof. Since $f_{s}(A, B) \neq 0$, then the matrix $G(A, B)=\left(\mathbf{z}_{1}, \ldots, \mathbf{z}_{l}\right)$ is not singular. If $(S, T) \in \mathbb{S t a b}_{\mathbb{S} L}(A, B)$, then

$$
G((S, T) \circ(A, B))=\left(\operatorname{det} S^{-1}\right)^{q}(\operatorname{det} T)^{p-1} G(A, B) \cdot \wedge^{s} T=G(A, B) .
$$

By virtue of invertibility of the matrix $G(A, B)$, we get that $\wedge^{s} T=\lambda E_{s}$, where $E_{s}$ is the identity matrix of order $l=\operatorname{Bin}(m, s)$. Note that the homomorphism $T \rightarrow \wedge^{s} T$ has the identity core $\left\{\zeta_{s} E_{m}\right\}$, where $\zeta_{s}=\sqrt[s]{1}$. Therefore, $\lambda=\zeta_{s}$, 
$S=\zeta_{s} E_{n}$ and $\operatorname{Stab}_{\mathbb{S} L}(A, B)$ consists of the finite number $s$ of pairs matrices $\left(\zeta_{s} E_{n}, \zeta_{s} E_{m}\right)$. Therefore, all points of the invariant set $\mathbb{M}_{f_{s}} \in \mathbb{C}^{n \times(n+m)}$ such that $f_{s}(A, B) \neq 0$ are regular and, consequently, its points are stable.

Theorem 6.2. Let $\operatorname{disc}(A) \neq 0$. Suppose also that $A=\operatorname{diag}\left(() \lambda_{1}, \ldots, \lambda_{n}\right)$ is the diagonal matrix in some base of space $\mathbb{C}^{n}$, and in the same base of space $\mathbb{C}^{n}$ all minors of matrix $B$ are distinct from zero. Then system $(A, B)$ is stable.

Proof. In the case $(n, m)=(4,2)$ this theorem was proved in [24].

(a) Let $\phi: \mathbb{C}^{n \times(n+m)} \rightarrow \mathbb{C}^{n \times(n+m)}$ be a morphism of algebraic manifolds, which satisfies to the following condition:

$$
\forall(S, T) \in \mathbb{G} L \text { and } \forall(A, B) \in \mathbb{C}^{n \times(n+m)} \phi\left(S^{-1} A S, S^{-1} B T\right)=(S, T) \circ \phi(A, B) .
$$

Show that system $(A, B)$ is stable if and only if the system $\phi(A, B)$ is stable.

Let the system $\phi(A, B)$ be stable. Then there is an invariant opened set $\mathbb{M}_{f}$ is defined by an invariant $f(A, B)$ such that $f(\phi(A, B)) \neq 0$ and action of group $\mathbb{G} L$ on $\mathbb{M}_{f}$ is closed. Then the invariant $\phi(f)$ determines the invariant open set $\mathbb{M}_{\phi(f)} \subset \mathbb{C}^{n \times(n+m)}$, on which $\mathbb{G} L$ acts with closed orbits. The inverse assertion can be got if instead of the isomorphism $\phi$ to consider the inverse morphism $\phi^{-1}$.

(b) Now we consider an automorphism $\phi_{\lambda}: \mathbb{C}^{n \times(n+m)} \rightarrow \mathbb{C}^{n \times(n+m)}$, which is given by the rule: $\phi_{\lambda}(A, B)=(A+\lambda E, B)$, where $E$ is the identity matrix. (It is easily to check that $\phi_{\lambda}$ satisfies to condition (6.1).)

It is obvious that $A+\lambda E=\operatorname{diag}\left(() \lambda_{1}+\lambda, \ldots, \lambda_{n}+\lambda\right)$ and $\operatorname{disc}(A+\lambda E) \neq 0$. Then there is a number $\lambda \in \mathbb{C}$ such that $\operatorname{det}(A+\lambda E) \neq 0$. (It is enough to take $\lambda \neq-\lambda_{i}, i=1, \ldots, n$.)

Let a system $\left(\wedge^{m} A, \wedge^{m} B\right)$ be an $m$-exterior degree of the system $(A, B)$. Assume that for the system $\left(\wedge^{m} A, \wedge^{m} B\right)$ the following condition

$$
\operatorname{rank}\left(\wedge^{m} B, \wedge^{m}(A B), \ldots, \wedge^{m}\left(A^{n-1} B\right)\right)<\operatorname{Bin}(n, m)
$$

is fulfilled. (The system $\left(\wedge^{m} A, \wedge^{m} B\right)$ is not complete controllable.)

It means that under the conditions of Theorem 6.2 there exist collections of indexes $\left(i_{1}, \ldots, i_{m}\right)$ and $\left(j_{1}, \ldots, j_{m}\right)$, where $1 \leq i_{1}<\ldots<i_{m} \leq n$ and $1 \leq j_{1}<\ldots<$ $j_{m} \leq n$ such that

$$
\left\{\left(i_{1}, \ldots, i_{m}\right)\right\} \cap\left\{\left(j_{1}, \ldots, j_{m}\right)\right\}=\varnothing \text { and } \lambda_{i_{1}} \cdot \ldots \cdot \lambda_{i_{m}}=\lambda_{j_{1}} \cdot \ldots \cdot \lambda_{j_{m}} .
$$

Then there exists a number $\lambda \in \mathbb{C}$ such that for the matrix $A+\lambda E$ condition (6.2) is not fulfilled.

Indeed, otherwise for all $\lambda$

$$
\left(\lambda_{i_{1}}+\lambda\right) \cdot \ldots \cdot\left(\lambda_{i_{m}}+\lambda\right)=\left(\lambda_{j_{1}}+\lambda\right) \cdot \ldots \cdot\left(\lambda_{j_{m}}+\lambda\right) .
$$

Equality (6.3) means that

$$
h_{k}\left(\lambda_{i_{1}}, \ldots, \lambda_{i_{m}}\right)=h_{k}\left(\lambda_{j_{1}}, \ldots, \lambda_{j_{m}}\right)
$$


for any elementary symmetric polynomials $h_{k}\left(\lambda_{i_{1}}, \ldots, \lambda_{i_{m}}\right), k=1, \ldots, m$ [15]. However, it is impossible since $\lambda_{i} \neq \lambda_{j}$ at $i \neq j$.

Further, since the set of all numbers $\lambda$ such that

$$
\operatorname{disc}\left(\wedge^{m}(A+\lambda E)\right) \neq 0 \text { and } \operatorname{det} A \neq 0
$$

is open in the Zariski topology and the Euclidean topology of space $\mathbb{C}$ [44], then their intersection is not empty. Consequently, there will be numbers $\lambda \in \mathbb{C}$, for which both conditions (6.4) are valid simultaneously. Then the system $\phi_{\lambda}(A, B)$ is stable and, in obedience to the item (a), the system $(A, B)$ will be also stable. It completes proof of item (b) and all Theorem 6.2.

Theorem 6.3. System $(A, B)$ of type $(n, m)$ is stable with respect to action (2.3) of group $\mathbb{G} L$ if and only if it is stable with respect to action (2.3) of group $\mathbb{S} L$.

Proof. We will consider the morphism of algebraic manifolds:

$$
\begin{gathered}
\phi: \mathbb{C}^{n \times(n+m)} \rightarrow \mathbb{C}^{n \times n} \times \mathbb{C}^{N}, N=\operatorname{Bin}(n, m), \\
\phi(A, B)=\left(A, \wedge^{m} B\right) .
\end{gathered}
$$

Notice that the image of morphism $\phi$ is a close variety in space $\mathbb{C}^{n \times n} \times \mathbb{C}^{N}$ and orbits of group $\mathbb{S} L$ the morphism $\phi$ transfers in the orbits of group $\mathbb{G} L(n, \mathbb{C})$ in space $\mathbb{C}^{n \times(n+m)}$ at action

$$
\begin{gathered}
\mathbb{S} L(n, \mathbb{C}) \times \mathbb{C}^{n \times n} \times \mathbb{C}^{N} \rightarrow \mathbb{C}^{n \times n} \times \mathbb{C}^{N}, \\
\left(S, A, \wedge^{m} B\right)=\left(S^{-1} A S, \wedge^{m}\left(S^{-1} B\right)\right) .
\end{gathered}
$$

It completes the proof.

\subsection{Stability of systems of type $(4,2)$}

In this subsection we show difficulties, which can arise up at construction of the set of all stable systems in case if $m$ is a divisor of $n$ (see [24]).

First of all, we show that if system $(A, B)$ is stable then the matrix $A$ is cyclic [15]. For this purpose we present the various Jordan formes of noncyclic matrix of order 4 :
a) $\left(\begin{array}{ll}\alpha_{1} & \\ & \alpha \\ 0 & \end{array}\right.$
$\left.\begin{array}{cc} & 0 \\ \alpha_{2} & \\ & \alpha_{3}\end{array}\right)$
b) $\left(\begin{array}{ll}\alpha_{1} & 1 \\ & \alpha \\ & \end{array}\right.$
$\left.\left.\begin{array}{cc}\alpha_{1} & \\ & \alpha_{2}\end{array}\right), c\right)\left(\begin{array}{c}\alpha_{1} \\ \\ 0\end{array}\right.$
$\left.\begin{array}{ccc}1 & & 0 \\ \alpha_{1} & & \\ & \alpha_{1} & 1 \\ & & \alpha_{1}\end{array}\right)$,
d) $\left(\begin{array}{ccc}\alpha_{1} & 1 & \\ & \alpha_{1} & 1 \\ & & \alpha_{1}\end{array}\right.$
$\left.\left.\begin{array}{c}0 \\ \alpha_{1}\end{array}\right), e\right)\left(\begin{array}{c}\alpha_{1} \\ 0\end{array}\right.$
$\alpha_{1}$
$\left.\left.\begin{array}{c}0 \\ \alpha_{2}\end{array}\right), f\right)\left(\begin{array}{l}\alpha_{1} \\ 0\end{array}\right.$
$\alpha_{1}$
$\left.\begin{array}{c}0 \\ \alpha_{1}\end{array}\right)$. 
Thus, there are six such forms.

Consider, for example, the system $(A, B)$ of type $(4,2)$, for which matrix $A$ looks like a) and matrix $B$ is arbitrary. Apply to $(A, B)$ the transformation $(S, T) \in \mathbb{G} L$, where

$$
S=\left(\begin{array}{llll}
\alpha & \beta & & 0 \\
\gamma & \delta & & \\
& & \nu & \\
0 & & & \mu
\end{array}\right)
$$

and numbers $\alpha, \beta, \gamma, \delta, \mu$, and $\nu$ satisfy a unique condition: $\operatorname{det} S \neq 0$. Then system $(A, B)$ can be transformed to the following system:

$$
S^{-1} A S=\left(\begin{array}{cccc}
\alpha_{1} & & & 0 \\
& \alpha_{1} & & \\
& & \alpha_{2} & \\
0 & & & \alpha_{3}
\end{array}\right), S^{-1} B T=\left(\begin{array}{cc}
0 & * \\
1 & * \\
1 & * \\
0 & *
\end{array}\right),
$$

where by character $*$ arbitrary numbers are marked.

From here it follows that the system $(A, B)$ contains a subsystem of type $(2,1)$. Consequently, according to Theorem 4.1 , the system $(A, B)$ is nonstable. By applying the same arguments to one of matrices b) - f), we obtain a similar result: the conditions of Theorem 4.1 are not valid.

We reduce the matrix $A$ of system $(A, B)$ to triangular form [15]. Then analysis of all possible Jordan forms of $A$ results in the conclusion: if the system is not stable then there exists a minor of the second order of matrix $B$, which equal to zero. Otherwise, if the system $(A, B)$ is stable, then there is even one nonzero minor of the second order of matrix $B$.

Denote by $I_{0}=\operatorname{det}(B, B) \equiv 0, I_{1}=\operatorname{det}(B, A B), I_{2}=\operatorname{det}\left(B, A^{2} B\right), a_{1}, \ldots, a_{4}$ invariants of system $(A, B)$.

Theorem 6.4. Let $(A, B)$ be a system of type $(4,2)$. Then the set $\mathbb{F}_{s}$ of all $\mathbb{S} L$ stable systems with respect to action (2.3) is determined by the condition:

$$
\mathbb{F}_{s}=\left\{(A, B) \in \mathbb{C}^{4 \times 6} \mid f_{s}(A, B) \neq 0\right\},
$$

where the polynomial invariant

$$
f_{s}(A, B)=\left(a_{1} a_{2} a_{3}-a_{3}^{2}-a_{1}^{2} a_{4}\right) I_{1}^{3}-\left(a_{1} a_{3}+a_{2}^{2}-4 a_{4}\right) I_{1}^{2} I_{2}+2 a_{2} I_{1} I_{2}^{2}-I_{2}^{3}
$$

has degree 24 with respect to elements $a_{i j}$ and $b_{i k}$ of matrices $A$ and $B$.

Proof. Assume that for the matrix $A$, we have $\operatorname{disc}(A) \neq 0$. In this case the system $(A, B)$ of type $(4,2)$ may be transformed to the following aspect:

$$
A=\left(\begin{array}{cccc}
\alpha_{1} & & & 0 \\
& \alpha_{2} & & \\
& & \alpha_{3} & \\
0 & & & \alpha_{4}
\end{array}\right), B=\left(\begin{array}{ll}
b_{11} & b_{12} \\
b_{21} & b_{22} \\
b_{31} & b_{32} \\
b_{41} & b_{42}
\end{array}\right) \text {. }
$$


Denote by $\wedge^{2} B=\left(\Delta_{12}, \Delta_{13}, \Delta_{14}, \Delta_{23}, \Delta_{24}, \Delta_{34}\right)^{T}$ a bivector of matrix $B$; here $\Delta_{i j}=b_{i 1} b_{j 2}-b_{i 2} b_{j 1}$ are minors of matrix $B ; i, j=1, \ldots, 4 ; i<j$. Then the invariants $I_{0}, I_{1}$, and $I_{2}$ of system $(A, B)$ are given by the formulas

$$
\left(\begin{array}{c}
0 \\
I_{1} \\
I_{2}
\end{array}\right)=\left(\begin{array}{ccc}
2 & 2 & 2 \\
\alpha_{1} \alpha_{2}+\alpha_{3} \alpha_{4} & \alpha_{1} \alpha_{3}+\alpha_{2} \alpha_{4} & \alpha_{1} \alpha_{4}+\alpha_{2} \alpha_{3} \\
\alpha_{1}^{2} \alpha_{2}^{2}+\alpha_{3}^{2} \alpha_{4}^{2} & \alpha_{1}^{2} \alpha_{3}^{2}+\alpha_{2}^{2} \alpha_{4}^{2} & \alpha_{1}^{2} \alpha_{4}^{2}+\alpha_{2}^{2} \alpha_{3}^{2}
\end{array}\right) \cdot\left(\begin{array}{c}
\Delta_{12} \Delta_{34} \\
-\Delta_{13} \Delta_{24} \\
\Delta_{14} \Delta_{23}
\end{array}\right) \text {. }
$$

The elementary computations show that

$$
\operatorname{det}\left(\begin{array}{ccc}
2 & 2 & 2 \\
\alpha_{1} \alpha_{2}+\alpha_{3} \alpha_{4} & \alpha_{1} \alpha_{3}+\alpha_{2} \alpha_{4} & \alpha_{1} \alpha_{4}+\alpha_{2} \alpha_{3} \\
\alpha_{1}^{2} \alpha_{2}^{2}+\alpha_{3}^{2} \alpha_{4}^{2} & \alpha_{1}^{2} \alpha_{3}^{2}+\alpha_{2}^{2} \alpha_{4}^{2} & \alpha_{1}^{2} \alpha_{4}^{2}+\alpha_{2}^{2} \alpha_{3}^{2}
\end{array}\right)=2(\operatorname{disc}(A))^{1 / 2} .
$$

Let $p_{1}=\alpha_{1} \alpha_{2}+\alpha_{3} \alpha_{4}, p_{2}=\alpha_{1} \alpha_{3}+\alpha_{2} \alpha_{4}$, and $p_{3}=\alpha_{1} \alpha_{4}+\alpha_{2} \alpha_{3}$. Since we consider that $\operatorname{disc}(A) \neq 0$, then from (6.5) we have

$$
\begin{gathered}
\left(\begin{array}{c}
\Delta_{12} \Delta_{34} \\
-\Delta_{13} \Delta_{24} \\
\Delta_{14} \Delta_{23}
\end{array}\right)=\left(\begin{array}{ccc}
2 & 2 & 2 \\
\alpha_{1} \alpha_{2}+\alpha_{3} \alpha_{4} & \alpha_{1} \alpha_{3}+\alpha_{2} \alpha_{4} & \alpha_{1} \alpha_{4}+\alpha_{2} \alpha_{3} \\
\alpha_{1}^{2} \alpha_{2}^{2}+\alpha_{3}^{2} \alpha_{4}^{2} & \alpha_{1}^{2} \alpha_{3}^{2}+\alpha_{2}^{2} \alpha_{4}^{2} & \alpha_{1}^{2} \alpha_{4}^{2}+\alpha_{2}^{2} \alpha_{3}^{2}
\end{array}\right)^{-1} \cdot\left(\begin{array}{c}
0 \\
I_{1} \\
I_{2}
\end{array}\right) \\
=\frac{1}{(2 \operatorname{disc}(A))^{1 / 2}}\left(\begin{array}{ccc}
0.5 p_{2} p_{3}\left(p_{2}-p_{3}\right) & \left(p_{2}^{2}-p_{3}^{2}\right) & \left(p_{2}-p_{3}\right) \\
0.5 p_{1} p_{3}\left(p_{1}-p_{3}\right) & \left(p_{1}^{2}-p_{3}^{2}\right) & \left(p_{1}-p_{3}\right) \\
0.5 p_{1} p_{2}\left(p_{1}-p_{2}\right) & \left(p_{1}^{2}-p_{2}^{2}\right) & \left(p_{1}-p_{2}\right)
\end{array}\right) \cdot\left(\begin{array}{c}
0 \\
I_{1} \\
I_{2}
\end{array}\right) \\
=\frac{1}{(2 \operatorname{disc}(A))^{1 / 2}}\left(\begin{array}{c}
\left.-\left(p_{2}-p_{3}\right)\left(p_{2}+p_{3}\right) I_{1}+\left(p_{2}-p_{3}\right) I_{2}\right) \\
\left.-\left(p_{1}-p_{3}\right)\left(p_{1}+p_{3}\right) I_{1}+\left(p_{1}-p_{3}\right) I_{2}\right) \\
\left.-\left(p_{1}-p_{2}\right)\left(p_{1}+p_{2}\right) I_{1}+\left(p_{1}-p_{2}\right) I_{2}\right)
\end{array}\right) .
\end{gathered}
$$

From the last relations we get

$$
\begin{gathered}
\Delta_{12} \Delta_{13} \Delta_{14} \Delta_{23} \Delta_{24} \Delta_{34} \\
=-\frac{1}{8 \operatorname{disc}(A)}\left(-\left(p_{2}+p_{3}\right) I_{1}+I_{2}\right)\left(-\left(p_{1}+p_{3}\right) I_{1}+I_{2}\right)\left(-\left(p_{1}+p_{2}\right) I_{1}+I_{2}\right) \\
=-\frac{-\left(p_{1}+p_{2}\right)\left(p_{1}+p_{3}\right)\left(p_{2}+p_{3}\right) I_{1}^{3}}{8 \operatorname{disc}(A)} \\
-\frac{\left(\left(p_{1}+p_{3}\right)\left(p_{2}+p_{3}\right)+\left(p_{1}+p_{3}\right)\left(p_{1}+p_{2}\right)+\left(p_{1}+p_{2}\right)\left(p_{2}+p_{3}\right)\right) I_{1}^{2} I_{2}}{8 \operatorname{disc}(A)} \\
+\frac{2\left(p_{1}+p_{2}+p_{3}\right) I_{1} I_{2}^{2}-I_{2}^{3}}{8 \operatorname{disc}(A)} \\
=-\frac{-\left(a_{1} a_{2} a_{3}-a_{3}^{2}-a_{1}^{2} a_{4}\right) I_{1}^{3}+\left(a_{1} a_{3}+a_{2}^{2}-4 a_{4}\right) I_{1}^{2} I_{2}-2 a_{2} I_{1} I_{2}^{2}+I_{2}^{3}}{8 \operatorname{disc}(A)}
\end{gathered}
$$


Above it was noted that the system $(A, B)$ would be not stable if either even one minor $\Delta_{i j}=0$ or $\operatorname{disc}(A)=0$. Then from (6.6) it follows that $(A, B)$ is $\mathbb{S} L$-stable if the invariant

$$
f_{s}(A, B)=\left(a_{1} a_{2} a_{3}-a_{3}^{2}-a_{1}^{2} a_{4}\right) I_{1}^{3}-\left(a_{1} a_{3}+a_{2}^{2}-4 a_{4}\right) I_{1}^{2} I_{2}+2 a_{2} I_{1} I_{2}^{2}-I_{2}^{3} \neq 0 .
$$

Consider the cyclic matrix

$$
\left(\begin{array}{cccc}
\alpha_{1} & & & 0 \\
& \alpha_{2} & & \\
& & \alpha_{3} & \\
0 & & & \alpha_{4}
\end{array}\right)
$$

Apply to this matrix the transformation

$$
S=\left(\begin{array}{cccc}
1 & 1 /\left(\alpha_{1}-\alpha_{2}\right) & & 0 \\
& 1 & & \\
0 & & 1 & \\
& & & 1
\end{array}\right)
$$

then, we have

$$
S^{-1} A S=\left(\begin{array}{cccc}
\alpha_{1} & 1 & & 0 \\
& \alpha_{2} & & \\
& & \alpha_{3} & \\
0 & & & \alpha_{4}
\end{array}\right) .
$$

Let $T=E$ be the identity matrix of order 2 . Then

$$
f_{s}\left(S^{-1} A S, S^{-1} B T\right)=(\operatorname{det} S)^{l_{S}}(\operatorname{det} T)^{l_{T}} f_{s}(A, B)=f_{s}(A, B) .
$$

Since we have

$$
\lim _{\left(\alpha_{1}-\alpha_{2}\right) \rightarrow 0} S^{-1} A S=\left(\begin{array}{cccc}
\alpha_{1} & 1 & & 0 \\
& \alpha_{1} & & \\
& & \alpha_{3} & \\
0 & & & \alpha_{4}
\end{array}\right),
$$

then even if $\left(\alpha_{1}-\alpha_{2}\right) \rightarrow 0$ the invariant polynomial $f_{s}(A, B)$ is saved.

Applying similar methods, it is possible to get from matrix (6.7) an arbitrary cyclic matrix $A$, for which $\operatorname{disc}(A)=0$. (Note that if matrix (6.7) is noncyclic, then the last assertion is incorrect.) Consequently, the polynomial $f_{s}(A, B)$ saves and for cyclic matrices $A$ such that $\operatorname{disc}(A)=0$.

Finally, we note that for noncyclic matrices $A$ system $(A, B)$ type $(4,2)$ is not stable. The proof is finished.

\subsection{Stability of systems of type $(m+1, m), m>1$}

(a) $m=2$. Let $A=\operatorname{diag}\left(() \alpha_{1}, \alpha_{2}, \alpha_{3}\right), \wedge^{2} B=\left(\Delta_{12}, \Delta_{13}, \Delta_{23}\right)^{T}$, and $\operatorname{disc}(A) \neq$ 0 . We take advantage of Theorems 5.1 and 6.1. 
Consider the invariant $I(A, B)=\operatorname{det} R(A, B)$, where $R(A, B)$ is matrix (5.3) at $n=3$ and $m=2$. Then we have

$$
\begin{gathered}
I(A, B) \\
=\operatorname{det}\left(\begin{array}{cc}
\alpha_{1} \Delta_{23} b_{11}-\alpha_{2} \Delta_{13} b_{21}+\alpha_{3} \Delta_{12} b_{31} & \alpha_{1} \Delta_{23} b_{12}-\alpha_{2} \Delta_{13} b_{22}+\alpha_{3} \Delta_{12} b_{32} \\
\alpha_{1}^{2} \Delta_{23} b_{11}-\alpha_{2}^{2} \Delta_{13} b_{21}+\alpha_{3}^{2} \Delta_{12} b_{31} & \alpha_{1}^{2} \Delta_{23} b_{12}-\alpha_{2}^{2} \Delta_{13} b_{22}+\alpha_{3}^{2} \Delta_{12} b_{32}
\end{array}\right) \\
=\left(\alpha_{1} \alpha_{2}\left(\alpha_{1}-\alpha_{2}\right)-\alpha_{1} \alpha_{3}\left(\alpha_{1}-\alpha_{3}\right)+\alpha_{2} \alpha_{3}\left(\alpha_{2}-\alpha_{3}\right)\right) \Delta_{12} \Delta_{13} \Delta_{23} \\
=\left(\alpha_{1}-\alpha_{2}\right)\left(\alpha_{1}-\alpha_{3}\right)\left(\alpha_{2}-\alpha_{3}\right) \Delta_{12} \Delta_{13} \Delta_{23} .
\end{gathered}
$$

It is clear that $I(A, B)$ is $\mathbb{G} L$-invariant, but it is not $\mathbb{S} L$-invariant. Therefore, we have to take the invariant

$$
I^{2}(A, B)=\operatorname{disc}(A) \Delta_{12}^{2} \Delta_{13}^{2} \Delta_{23}^{2} .
$$

Now we must show that if the matrix $A$ is noncyclic then $I^{2}(A, B)=0$. It can be checked by the methods of subsection 6.1 .

Thus, the set of all $\mathbb{S} L$-stable systems $(A, B)$ of type $(3,2)$ is given by the condition

$$
\mathbb{F}_{s}=\left\{(A, B) \in \mathbb{C}^{3 \times 5} \mid I^{2}(A, B) \neq 0\right\} .
$$

(b) $m>2$. A proof of this case word for a word repeats the previous proof. In this case we get

$$
I^{2}(A, B)=\operatorname{disc}(A) \underbrace{\Delta_{1 \ldots m}^{2} \cdot \ldots \cdot \Delta_{2 \ldots m+1}^{2}}_{m+1} .
$$

Therefore, the set of all $\mathbb{S} L$-stable systems $(A, B)$ of type $(m+1, m)$ is given by the condition

$$
\mathbb{F}_{s}=\left\{(A, B) \in \mathbb{C}^{(m+1) \times(2 m+1)} \mid I^{2}(A, B) \neq 0\right\}
$$

\section{Description of ring of invariants for system $(A, B)$}

\subsection{Structure of invariants of group $\mathbb{S} L$ for system $(A, B)$}

Denote by $a_{1}, \ldots, a_{n}$ the coefficients of the characteristic polynomial of matrix $A$. Let $f(A, B) \in \mathbb{C}[A, B]^{\mathbb{S} L}$ be an invariant of group $\mathbb{S} L=\mathbb{S} L(n, \mathbb{C}) \times \mathbb{S} L(m, \mathbb{C})$ with respect to action (2.3). Let also the vector $\left(\Delta_{1}, \ldots, \Delta_{r}\right)^{T}=\wedge^{m}(B)$ be the exterior degree of matrix $B ; r=\operatorname{Bin}(n, m)$.

Theorem 7.1. Any polynomial $\mathbb{S} L$-invariant of group $\mathbb{S} L$ is a function of elements $a_{1}, \ldots, a_{n}, \Delta_{1}, \ldots, \Delta_{r}$. 
Proof. We represent the polynomial $f(A, B)$ in the following form:

$$
f(A, B)=\sum_{i=1}^{r} g_{i}(A) \otimes v_{i}(B)
$$

where $g_{i}(A)\left(v_{i}(B)\right)$ are polynomials depending only on the elements of matrix $A$ (the elements of matrix $B$ ) and the polynomials $g_{i}(A)$ it is possible to choose linearly independent.

Further, $\forall T \in \mathbb{S} L(m, \mathbb{C})$, we have

$$
\sum_{i=1}^{r} g_{i}(A) \otimes v_{i}(B)=f(A, B)=f(A, B T)=\sum_{i=1}^{r} g_{i}(A) \otimes v_{i}(B T) .
$$

Consequently, from here it follows that $\sum_{i=1}^{r} g_{i}(A) \otimes\left[v_{i}(B)-v_{i}(B T)\right]=0$.

By virtue of the linear independence of polynomials $g_{i}(A)$ over $\mathbb{C}$, we get that $\forall i$ and for all $T \in \mathbb{S} L(m, \mathbb{C}) v_{i}(B)=v_{i}(B T)$. In other words, the inclusion $v_{i}(B) \in$ $\mathbb{C}[B]^{\mathbb{S} L(m, \mathbb{C})}=\mathbb{C}\left[\Delta_{1}, \ldots, \Delta_{r}\right]$ takes place. Consequently, $f(A, B)=h\left(A, \Delta_{1}, \ldots, \Delta_{r}\right)$ is a polynomial of elements of matrix $A$ and coordinates of polyvector $\wedge^{m} B$. Thus, $\forall(S, T) \in \mathbb{S} L$ we have

$$
(S, T) \circ f(A, B)=\left(S^{-1} A S, S^{-1} B T\right)=h\left(A, \wedge^{m}\left(S^{-1} B T\right)\right)=h\left(A, \Delta_{1}, \ldots, \Delta_{r}\right),
$$

where $h(\ldots)$ is a homogeneous polynomial of elements of matrix $A$ and $\Delta_{1}, \ldots, \Delta_{r}$. Besides, if we take into account that any $\mathbb{S} L(n, \mathbb{C})$-invariant of matrix $A$ is a polynomial of elements $a_{1}, \ldots, a_{n}$, then the invariant $f(A, B)$ is the function of $a_{1}, \ldots, a_{n}, \Delta_{1}, \ldots, \Delta_{r}$.

\subsection{Ring of invariants for system $(A, B)$ of type $(2 p, 2), p>1$}

Theorem 7.2. Let $I_{1}(A, B), \ldots, I_{2 p-2}(A, B)$ be invariants (5.8). Then the following equality

$$
\mathbb{C}[A, B]^{\mathbb{S} L}=\mathbb{C}\left[a_{1}, \ldots, a_{2 p}, I_{1}, \ldots, I_{2 p-2}\right]
$$

takes place. Moreover, the number $4 p-2$ of generators of ring $\mathbb{C}[A, B]^{\mathbb{S L}}$ is minimal.

Proof. (a) $p=2$. In this case the proof easily can be got from the proofs of Theorems 5.2 and 6.4.

(b) $p=3$. Let $A=\operatorname{diag}\left(() \alpha_{1}, \alpha_{2}, \alpha_{3}, \alpha_{4}, \alpha_{5}, \alpha_{6}\right), B=\left(b_{1}, b_{2}\right) \in \mathbb{C}^{5 \times 2}, \wedge^{2} B=$ $\left(\Delta_{12}, \Delta_{13}, \ldots, \Delta_{56}\right)^{T}$, and $\operatorname{disc}(A) \neq 0$.

Represent the equivalence $\operatorname{det}(B, B, B) \equiv 0$ in the following form:

$$
\begin{gathered}
\operatorname{det}(B, B, B)=\Delta_{12} \Delta_{34} \Delta_{56}-\Delta_{13} \Delta_{25} \Delta_{46}+\Delta_{14} \Delta_{26} \Delta_{45} \\
-\Delta_{15} \Delta_{24} \Delta_{36}+\Delta_{16} \Delta_{35} \Delta_{24} \equiv 0 .
\end{gathered}
$$


Construct invariants (5.8):

$$
\left(\begin{array}{c}
I_{0}=\operatorname{det}(B, A B, A B) \\
I_{1}=\operatorname{det}\left(B, A B, A^{2} B\right) \\
I_{2}=\operatorname{det}\left(B, A B, A^{3} B\right) \\
I_{3}=\operatorname{det}\left(B, A B, A^{4} B\right) \\
I_{4}=\operatorname{det}\left(B, A B, A^{5} B\right)
\end{array}\right)=Q\left(\alpha_{1}, \ldots, \alpha_{6}\right) \cdot\left(\begin{array}{c}
\Delta_{12} \Delta_{34} \Delta_{56} \\
-\Delta_{13} \Delta_{25} \Delta_{46} \\
\Delta_{14} \Delta_{26} \Delta_{45} \\
-\Delta_{15} \Delta_{24} \Delta_{36} \\
\Delta_{16} \Delta_{35} \Delta_{24}
\end{array}\right),
$$

where $I_{0} \equiv 0$ and

$$
\begin{aligned}
& Q\left(\alpha_{1}, \ldots, \alpha_{6}\right) \\
& =\alpha_{1} \cdot \ldots \cdot \alpha_{6} \cdot\left(\begin{array}{ccc}
\frac{1}{\alpha_{3} \alpha_{4}}+\frac{1}{\alpha_{1} \alpha_{2}}+\frac{1}{\alpha_{5} \alpha_{6}} & \cdots & \frac{1}{\alpha_{2} \alpha_{4}}+\frac{1}{\alpha_{3} \alpha_{5}}+\frac{1}{\alpha_{1} \alpha_{6}} \\
\vdots & \cdots & \vdots \\
\frac{\alpha_{5}^{4} \alpha_{6}^{4}}{\alpha_{3} \alpha_{4}}+\frac{\alpha_{3}^{4} \alpha_{4}^{4}}{\alpha_{1} \alpha_{2}}+\frac{\alpha_{1}^{4} \alpha_{2}^{4}}{\alpha_{5} \alpha_{6}} & \cdots & \frac{\alpha_{3}^{4} \alpha_{5}^{4}}{\alpha_{2} \alpha_{4}}+\frac{\alpha_{1}^{4} \alpha_{6}^{4}}{\alpha_{3} \alpha_{5}}+\frac{\alpha_{2}^{4} \alpha_{4}^{4}}{\alpha_{1} \alpha_{6}}
\end{array}\right) \in \mathbb{C}^{5 \times 5} .
\end{aligned}
$$

Assume that $\operatorname{det} Q\left(\alpha_{1}, \ldots, \alpha_{6}\right) \neq 0$. Let also $I_{1}=\ldots=I_{4}=0$. Then from (7.1) it follows that

$$
\Delta_{12} \Delta_{34} \Delta_{56}=\Delta_{13} \Delta_{25} \Delta_{46}=\Delta_{14} \Delta_{26} \Delta_{45}=\Delta_{15} \Delta_{24} \Delta_{36}=\Delta_{16} \Delta_{35} \Delta_{24}=0 .
$$

Researches of system (7.2) result in one of systems of equations: either $\Delta_{12}=$ $\Delta_{13}=\Delta_{14}=\Delta_{23}=\Delta_{24}=\Delta_{34}=0$ or $\ldots$ or $\Delta_{34}=\Delta_{35}=\Delta_{36}=\Delta_{45}=$ $\Delta_{46}=\Delta_{56}=0$. According to Theorem 5.3 it means that there exists a subsystem of type $(2,1)$ of system $(A, B)$. If it is assertion to complement by conditions $a_{1}=\ldots=a_{6}=0$, then we get that the system $(A, B)$ is the null-form.

Now we suppose that $g(A, B) \in \mathbb{C}[A, B]^{\mathbb{S} L}$ is the homogeneous polynomial invariant such that $g(A, B)=0$ in all roots of polynomials $a_{1}, \ldots ., a_{6}, I_{1}, \ldots, I_{4}$. Then by the known Hilbert theorem [35] there exists an integer number $d \geq 1$ such that $g^{d}(A, B) \in \mathbb{C}\left[a_{1}, \ldots, a_{6}, I_{1}, \ldots, I_{4}\right]$. Assume that for the polynomial invariant $g(A, B)$ such that $g(A, B)=0$, we have $d>1$. (In other words, $g(A, B) \notin$ $\left.\mathbb{C}\left[a_{1}, \ldots, a_{6}, I_{1}, \ldots, I_{4}\right].\right)$ It is clear that the invariants $I_{1}, \ldots, I_{4}$ are polynomials of degree 3 with respect to $\Delta_{12}, \ldots, \Delta_{56}$. In addition, degree 3 is the minimal degree with respect to $\Delta_{12}, \ldots, \Delta_{56}$ of all invariants depending on $B$ in the ring $\mathbb{C}[A, B]^{\mathbb{S} L}$. Therefore, there exists the invariant $g(A, B)$ such that $d=1$, and therefore, $\mathbb{C}\left[a_{1}, \ldots, a_{6}, I_{1}, \ldots, I_{4}\right]=\mathbb{C}[A, B]^{\mathbb{S} L}$.

(c) $p>3$. Let $A=\operatorname{diag}\left(() \alpha_{1}, \ldots, \alpha_{2 p}\right), \wedge^{2} B=\left(\Delta_{12}, \Delta_{13}, \ldots, \Delta_{2 p-1,2 p}\right)^{T}$, and $\operatorname{disc}(A) \neq 0$.

A proof of the case $p>3$ repeats the proof of Theorem 7.2 for case $p=3$. It is necessary only to do some generalizations. For $p>3$ system (7.1) has such form:

$$
\left(\begin{array}{c}
I_{0} \\
I_{1} \\
\vdots \\
I_{2 p-2}
\end{array}\right)=Q\left(\alpha_{1}, \ldots, \alpha_{2 p}\right) \cdot \underbrace{\left(\begin{array}{c}
\Delta_{12} \Delta_{34} \cdot \ldots \cdot \Delta_{2 p-1,2 p} \\
-\Delta_{13} \Delta_{25} \cdot \ldots \cdot \Delta_{2 p-2,2 p} \\
\vdots \\
(-1)^{l} \Delta_{i_{1} i_{2}} \Delta_{j_{1} j_{2}} \cdot \ldots \cdot \Delta_{k_{1} k_{2}}
\end{array}\right)}_{p \text { factors }}
$$


where $I_{0} \equiv 0, Q\left(\alpha_{1}, \ldots, \alpha_{2 p}\right) \in \mathbb{C}^{(2 p-1) \times(2 p-1)}, 1 \leq i_{1}<i_{2} \leq 2 p, \ldots, 1 \leq k_{1}<$ $k_{2} \leq 2 p, l=1, \ldots, 2 p-1$, and permutations $\left(i_{1}, i_{2}\right), \ldots,\left(k_{1}, k_{2}\right)$ are satisfied to the condition $\left(i_{1}, i_{2}, \ldots, k_{1}, k_{2}\right) \in\{1,2, \ldots, 2 p-1,2 p\}$. There are all $(2 p-1) ! !=$ $1 \cdot 3 \cdot 5 \cdot \ldots \cdot(2 p-1)$ permutations.

Further, let $I_{1}=\ldots=I_{2 p-2}=0$. If $\operatorname{det} Q\left(\alpha_{1}, \ldots, \alpha_{2 p}\right) \neq 0$, then from (7.3) it follows that $\Delta_{12} \Delta_{34} \cdot \ldots \cdot \Delta_{2 p-1,2 p}=\ldots=\Delta_{i_{1} i_{2}} \Delta_{j_{1} j_{2}} \cdot \ldots \cdot \Delta_{k_{1} k_{2}}=0$. It means that there exists a submatrix $B_{p-1} \in \mathbb{C}^{(p-1) \times 2}$ of matrix $B$ such that $\wedge^{2} B_{p-1}=0$. A further algorithm of proof is obvious.

Let us compute the dimension of space of orbits for system of type $(2 p, 2)$. From formula (4.1) it follows that for the system of type $(2 p, 2)$ we have $\operatorname{dim}_{\mathbb{C}}$ $\mathbb{O}^{\mathbb{S} L}(\mathbb{S})=4 p-2$. It means that in Theorem 7.2 the number of generators of ring $\mathbb{C}[A, B]^{\mathbb{S} L}$ is minimal.

\subsection{Ring of invariants for system $(A, B)$ of type $(2 p+1,2), p \geq 1$}

Theorem 7.3. Let

$$
I_{i j}(A, B)=\operatorname{det}\left(\begin{array}{cc}
\operatorname{det}\left(B, A B, \ldots, A^{p-1} B, A^{i} b_{1}\right) & \operatorname{det}\left(B, A B, \ldots, A^{p-1} B, A^{i} b_{2}\right) \\
\operatorname{det}\left(B, A B, \ldots, A^{p-1} B, A^{j} b_{1}\right) & \operatorname{det}\left(B, A B, \ldots, A^{p-1} B, A^{j} b_{2}\right)
\end{array}\right),
$$

$p \leq i<j \leq 2 p+1$, be invariants (5.11). Then the following equality

$$
\mathbb{C}[A, B]^{\mathbb{S} L}=\mathbb{C}[a_{1}, \ldots, a_{2 p+1}, \underbrace{I_{p, p+1}, \ldots, I_{p, 2 p}}_{p}, \underbrace{I_{p+1, p+2}, \ldots, I_{p+1,2 p}}_{p-1}]
$$

takes place. Moreover, the number $4 p$ of generators of ring $\mathbb{C}[A, B]^{\mathbb{S} L}$ is minimal.

Proof. (a) $p=2$. Let $A=\operatorname{diag}\left(() \alpha_{1}, \alpha_{2}, \alpha_{3}, \alpha_{4}, \alpha_{5}\right), B=\left(b_{1}, b_{2}\right) \in \mathbb{C}^{5 \times 2}, \wedge^{2} B=$ $\left(\Delta_{12}, \ldots, \Delta_{45}\right)^{T}$, and $\operatorname{disc}(A) \neq 0$.

Represent the functions $\operatorname{det}\left(B, B, b_{1}\right) \equiv 0$ and $\operatorname{det}\left(B, B, b_{2}\right) \equiv 0$ in the following forms:

$$
\begin{gathered}
\operatorname{det}\left(B, B, b_{1}\right) \\
=2 b_{11}\left(\Delta_{23} \Delta_{45}-\Delta_{24} \Delta_{35}+\Delta_{25} \Delta_{34}\right)-2 b_{21}\left(\Delta_{13} \Delta_{45}-\Delta_{14} \Delta_{35}+\Delta_{15} \Delta_{34}\right) \\
+2 b_{31}\left(\Delta_{12} \Delta_{45}-\Delta_{14} \Delta_{25}+\Delta_{24} \Delta_{15}\right)-2 b_{41}\left(\Delta_{12} \Delta_{35}-\Delta_{13} \Delta_{25}+\Delta_{23} \Delta_{15}\right) \\
+2 b_{51}\left(\Delta_{12} \Delta_{34}-\Delta_{13} \Delta_{24}+\Delta_{23} \Delta_{34}\right) \equiv 0
\end{gathered}
$$

and

$$
\operatorname{det}\left(B, B, b_{2}\right)
$$

$$
\begin{gathered}
=2 b_{12}\left(\Delta_{23} \Delta_{45}-\Delta_{24} \Delta_{35}+\Delta_{25} \Delta_{34}\right)-2 b_{22}\left(\Delta_{13} \Delta_{45}-\Delta_{14} \Delta_{35}+\Delta_{15} \Delta_{34}\right) \\
+2 b_{32}\left(\Delta_{12} \Delta_{45}-\Delta_{14} \Delta_{25}+\Delta_{24} \Delta_{15}\right)-2 b_{42}\left(\Delta_{12} \Delta_{35}-\Delta_{13} \Delta_{25}+\Delta_{23} \Delta_{15}\right) \\
+2 b_{52}\left(\Delta_{12} \Delta_{34}-\Delta_{13} \Delta_{24}+\Delta_{23} \Delta_{34}\right) \equiv 0 .
\end{gathered}
$$


Taking into account the structure of functions $\operatorname{det}\left(B, B, b_{1}\right), \operatorname{det}\left(B, B, b_{2}\right)$, we form the following zero $(2 \times 2)$-matrix:

$$
\begin{aligned}
& R_{0}=2\left(\begin{array}{lllll}
1 & -1 & 1 & -1 & 1 \\
1 & -1 & 1 & -1 & 1
\end{array}\right) \\
& \times\left(\begin{array}{cc}
b_{11}\left(\Delta_{23} \Delta_{45}-\Delta_{24} \Delta_{35}+\Delta_{25} \Delta_{34}\right) & b_{12}\left(\Delta_{23} \Delta_{45}-\Delta_{24} \Delta_{35}+\Delta_{25} \Delta_{34}\right) \\
b_{21}\left(\Delta_{13} \Delta_{45}-\Delta_{14} \Delta_{35}+\Delta_{15} \Delta_{34}\right) & b_{22}\left(\Delta_{13} \Delta_{45}-\Delta_{14} \Delta_{35}+\Delta_{15} \Delta_{34}\right) \\
b_{31}\left(\Delta_{12} \Delta_{45}-\Delta_{14} \Delta_{25}+\Delta_{24} \Delta_{15}\right) & b_{32}\left(\Delta_{12} \Delta_{45}-\Delta_{14} \Delta_{25}+\Delta_{24} \Delta_{15}\right) \\
b_{41}\left(\Delta_{12} \Delta_{35}-\Delta_{13} \Delta_{25}+\Delta_{23} \Delta_{15}\right) & b_{42}\left(\Delta_{12} \Delta_{35}-\Delta_{13} \Delta_{25}+\Delta_{23} \Delta_{15}\right) \\
b_{51}\left(\Delta_{12} \Delta_{34}-\Delta_{13} \Delta_{24}+\Delta_{23} \Delta_{34}\right) & b_{52}\left(\Delta_{12} \Delta_{34}-\Delta_{13} \Delta_{24}+\Delta_{23} \Delta_{34}\right)
\end{array}\right) .
\end{aligned}
$$

Now we use the structure of matrix $R_{0}$ for computation of invariants. Then we have:

$$
\begin{aligned}
I_{i j}(A, B) & =\operatorname{det}\left(\begin{array}{ccc}
\operatorname{det}\left(B, A B, A^{i} b_{1}\right) & \operatorname{det}\left(B, A B, A^{i} b_{2}\right) \\
\operatorname{det}\left(B, A B, A^{j} b_{1}\right) & \operatorname{det}\left(B, A B, A^{j} b_{2}\right)
\end{array}\right) \\
& =\operatorname{det}\left[\left(\begin{array}{lllll}
\alpha_{1}^{i} & -\alpha_{2}^{i} & \alpha_{3}^{i} & -\alpha_{4}^{i} & \alpha_{5}^{i} \\
\alpha_{1}^{j} & -\alpha_{2}^{j} & \alpha_{3}^{j} & -\alpha_{4}^{j} & \alpha_{5}^{j}
\end{array}\right) \times\right.
\end{aligned}
$$

$\operatorname{diag}\left(()\left(\alpha_{2} \alpha_{3}+\alpha_{4} \alpha_{5}\right) \Delta_{23} \Delta_{45}-\left(\alpha_{2} \alpha_{4}+\alpha_{3} \alpha_{5}\right) \Delta_{24} \Delta_{35}+\left(\alpha_{2} \alpha_{5}+\alpha_{3} \alpha_{4}\right) \Delta_{25} \Delta_{34}\right.$,

$$
\begin{gathered}
\left(\alpha_{1} \alpha_{3}+\alpha_{4} \alpha_{5}\right) \Delta_{13} \Delta_{45}-\left(\alpha_{1} \alpha_{4}+\alpha_{3} \alpha_{5}\right) \Delta_{14} \Delta_{35}+\left(\alpha_{1} \alpha_{5}+\alpha_{3} \alpha_{4}\right) \Delta_{15} \Delta_{34} \\
\left(\alpha_{1} \alpha_{2}+\alpha_{4} \alpha_{5}\right) \Delta_{12} \Delta_{45}-\left(\alpha_{1} \alpha_{4}+\alpha_{2} \alpha_{5}\right) \Delta_{14} \Delta_{25}+\left(\alpha_{2} \alpha_{4}+\alpha_{1} \alpha_{5}\right) \Delta_{24} \Delta_{15} \\
\left(\alpha_{1} \alpha_{2}+\alpha_{3} \alpha_{5}\right) \Delta_{12} \Delta_{35}-\left(\alpha_{1} \alpha_{3}+\alpha_{2} \alpha_{5}\right) \Delta_{13} \Delta_{25}+\left(\alpha_{2} \alpha_{3}+\alpha_{1} \alpha_{5}\right) \Delta_{23} \Delta_{15} \\
\left.\left.\left(\alpha_{1} \alpha_{2}+\alpha_{3} \alpha_{4}\right) \Delta_{12} \Delta_{34}-\left(\alpha_{1} \alpha_{3}+\alpha_{2} \alpha_{4}\right) \Delta_{13} \Delta_{24}+\left(\alpha_{2} \alpha_{3}+\alpha_{3} \alpha_{4}\right) \Delta_{23} \Delta_{34}\right) \cdot B\right] .
\end{gathered}
$$

We represent system (7.4) in such aspect:

$$
\left(\begin{array}{c}
0 \\
\vdots \\
0 \\
I_{23} \\
I_{24} \\
I_{34}
\end{array}\right)=\wedge^{2}\left(\left(\begin{array}{ccccc}
1 & -1 & 1 & -1 & 1 \\
\alpha_{1} & -\alpha_{2} & \alpha_{3} & -\alpha_{4} & \alpha_{5} \\
\alpha_{1}^{2} & -\alpha_{2}^{2} & \alpha_{3}^{2} & -\alpha_{4}^{2} & \alpha_{5}^{2} \\
\alpha_{1}^{3} & -\alpha_{2}^{3} & \alpha_{3}^{3} & -\alpha_{4}^{3} & \alpha_{5}^{3} \\
\alpha_{1}^{4} & -\alpha_{2}^{4} & \alpha_{3}^{4} & -\alpha_{4}^{4} & \alpha_{5}^{4}
\end{array}\right) \cdot D \cdot B\right)
$$

where $D=\operatorname{diag}\left(() d_{1}, \ldots, d_{5}\right)$ is the diagonal matrix from system (7.4).

In order that system (7.5) had trivial solution only, it is necessary that $I_{23}=$ $I_{24}=I_{34}=0$. Indeed, $\operatorname{since} \operatorname{disc}(A) \neq 0$, then from $(7.5)$ it follows that $\wedge^{2}(D \cdot B)=$ 0 . The last equality is possible if and only if

$$
d_{i} d_{j} \Delta_{i j}=0 ; 1 \leq i<j \leq 5
$$

The following variants are here possible. 
(a1) $d_{1} \neq 0, \ldots, d_{5} \neq 0$. Then we have $\wedge^{2} B=0$. It means $\Delta_{i j}=0 ; 1 \leq i<$ $j \leq 5$.

(a2) $d_{1}=0, d_{2} \neq 0, \ldots, d_{5} \neq 0$. Then $\wedge^{2} B_{1}=0$, where $B_{1}$ is the submatrix of matrix $B$ without the first row.

(a3) $d_{1}=d_{2}=0, d_{3} \neq 0, \ldots, d_{5} \neq 0$. Then $\wedge^{2} B_{12}=0$, where $B_{12}$ is the submatrix of matrix $B$ without the first and second rows.

In all other cases system (7.6) does not have solutions. From here it follows that in the space $\mathbb{X}$ there exists the space $\mathbb{X}_{1}$ such that $\operatorname{dim} \mathbb{X}_{1} \cap B(\mathbb{U})=1$ and $\operatorname{dim} \mathbb{X}_{1} / 1<5 / 2$. It means that the system $(A, B)$ is the null-form, which is defined by the invariants $a_{1}, \ldots, a_{5}, I_{23}, I_{24}, I_{34}$ (see Theorems 2.2 and 5.4 ).

(b) $p>2$. In order that to generalize the proof of Theorem 7.3 in this case, it is necessary to do alterations in the structure of diagonal matrix $D$ only. The diagonal elements of this matrix will be have the form

$$
d_{i}=\sum_{j=1}^{2 p-1}(-1)^{i} f_{i j}\left(\alpha_{1}, \ldots, \alpha_{2 p+1}\right) \underbrace{\Delta_{i_{1} i_{2}} \cdot \ldots \cdot \Delta_{j_{1} j_{2}}}_{p},
$$

where $\left\{i_{1}, i_{2}, \ldots, j_{1}, j_{2}\right\}=\{1, \ldots, 2 p+1\} ; 1 \leq i_{1}<i_{2} \leq 2 p+1, \ldots, 1 \leq j_{1}<j_{2} \leq$ $2 p+1 ; i=1, \ldots, 2 p+1$.

In order that the conditions of Theorem 2.1 were satisfied, it is necessary the equality to zero of $\operatorname{Bin}(p+1,2)=p(p+1) / 2$ invariants (5.11). However, among these invariants there exist $(p-1)(p-2) / 2$ syzygies [37,44].

Invariants (5.11) are coordinates of bivector

$$
\wedge^{2}\left(\begin{array}{cc}
\operatorname{det}\left(B, A B, \ldots, A^{p-1} B, A^{p} b_{1}\right) & \operatorname{det}\left(B, A B, \ldots, A^{p-1} B, A^{p} b_{2}\right) \\
\vdots & \vdots \\
\operatorname{det}\left(B, A B, \ldots, A^{p-1} B, A^{2 p} b_{1}\right) & \operatorname{det}\left(B, A B, \ldots, A^{p-1} B, A^{2 p} b_{2}\right)
\end{array}\right) .
$$

Thus, among generators of the ring $\mathbb{C}[A, B]^{\mathbb{S} L}$ there must be $p(p+1) / 2-(p-$ 1) $(p-2) / 2=2 p-1$ algebraically independent invariants (5.11). These invariants are indicated in Theorem 7.3.

Now we compute the dimension of space of orbits for system of type $(2 p+1,2)$.

From formula (4.1) it follows that for the system of type $(2 p+1,2)$ we have $\operatorname{dim}_{\mathbb{C}} \mathbb{O}^{\mathbb{S} L}(\mathbb{S})=4 p$. It means that in Theorem 7.3 the number of generators of ring $\mathbb{C}[A, B]^{\mathbb{S} L}$ is minimal.

\subsection{Ring of invariants for system $(A, B)$ of type $(n, m), n>m$}

As regards of a description of rings of invariants for systems of arbitrary types, we can state the following reasons.

Denote by $\mathbb{W}^{\circ} \subset \mathbb{S}$ an algebraic variety of all null-forms of space $\mathbb{S}$ with respect to action $(2.3)$ of group $\mathbb{S} L$. Let also $f_{1}(\cdot), \ldots, f_{k}(\cdot)$ be homogeneous invariant polynomials defining the variety $\mathbb{W}^{\circ}$. (The last means that if $\mathbb{W}_{\max }$ is the maximal subvariety in $\mathbb{S}$ such that $\forall y \in \mathbb{S}_{\max } f_{1}(y)=0, \ldots, f_{k}(y)=0$, then $\mathbb{W}_{\max }=\mathbb{W}^{\circ}$.) 
We will denote by $\mathbb{C}\left[f_{1}, \ldots, f_{k}\right]$ a ring of invariants generating subvariety $\mathbb{W}^{\circ}$. Then the following theorem holds true.

Theorem 7.4. (See [35]). $\mathbb{C}[\mathbb{S}]^{\mathbb{S} L}=\overline{\mathbb{C}\left[f_{1}, \ldots, f_{k}\right]}$, where the symbol $\overline{\mathbb{C}\left[f_{1}, \ldots, f_{k}\right]}$ means the integer closure of the ring $\mathbb{C}\left[f_{1}, \ldots, f_{k}\right] \subset \mathbb{C}[\mathbb{S}]^{\mathbb{S} L}$ in the ring $\mathbb{C}[\mathbb{S}]^{\mathbb{S} L}$.

Now we return to the equivalence problem. From Theorem 7.4 it follows that although rings $\mathbb{C}\left[f_{1}, \ldots, f_{k}\right]$ and $\mathbb{C}[\mathbb{S}]^{\mathbb{S} L}$ do not coincide, but their quotient fields coincide: $\mathbb{C}\left(f_{1}, \ldots, f_{k}\right)=\mathbb{C}(\mathbb{S})^{\mathbb{S} L}$. It means that invariants $f_{1}(\cdot), \ldots, f_{k}(\cdot)$ define a point $(A, B) \in \mathbb{S}$ to within a birational equivalence, and consequently, their knowledge completely gives the solution of equivalence problem for systems $\left(A_{1}, B_{1}\right) \in \mathbb{F}_{s} \subset \mathbb{S}$ and $\left(A_{2}, B_{2}\right) \in \mathbb{F}_{s} \subset \mathbb{S}$.

Since in general case the set $\mathbb{F}_{s}$ of stable systems is unknown, for the solution of the equivalence problem an open subset in $\mathbb{F}_{s}$, which is defined by the conditions of Theorem 6.2, it can be used.

For the decision of equivalence problem Theorem 7.4 can be used as follows. Assume that the homogeneous invariants $a_{1}(A), \ldots, a_{n}(A), I_{1}(A, B), \ldots, I_{r}(A, B)$ define the manifold $\mathbb{W}^{\circ} \subset \mathbb{S}$ of null-forms $(A, B)$ of type $(n, m)$. It means that polynomials $a_{1}(A), \ldots, a_{n}(A), I_{1}(A, B), \ldots, I_{r}(A, B)$ are generators of the quotient field $\mathbb{C}(A, B)^{\mathbb{S L}}$ of ring $\mathbb{C}[A, B]^{\mathbb{S L}}$.

Let $\mathcal{I}=\mathbb{C}\left[a_{1}, \ldots, a_{n}, I_{1}, \ldots, I_{r}\right] \subset \mathbb{C}[A, B]$ be an ideal, which is generated by the polynomials $a_{1}, \ldots, a_{n}, I_{1}, \ldots, I_{r}$ in the ring of polynomials from elements $a_{11}, \ldots, a_{n n}, b_{11}, \ldots, b_{n m}$ of matrices $A$ and $B$.

Let $g(A, B)$ be an polynomial in $\mathbb{C}[A, B]$. Assume that there exists an integer $k$ such that from the condition $g^{k}(A, B) \in \mathcal{I}$ it follows that $g(A, B) \in \mathcal{I}$.

Definition 7.1. (See $[35,44])$. The ideal $\mathcal{I} \in \mathbb{C}[A, B]$ is called a radical ideal if from the condition $g^{k}(A, B) \in \mathcal{I}$ it follows that $g(A, B) \in \mathcal{I}$.

Thus, in generic case we have $\mathcal{I}=\mathbb{C}\left[a_{1}, \ldots, a_{n}, I_{1}, \ldots, I_{r}\right] \subset \mathbb{C}[A, B]^{\mathbb{S} L}$. In order that the equality $\mathcal{I}=\mathbb{C}\left[a_{1}, \ldots, a_{n}, I_{1}, \ldots, I_{r}\right]=\mathbb{C}[A, B]^{\mathbb{S L}}$ takes place, the ideal $\mathcal{I}$ must be radical. (In this case, the conditions of Theorem 7.4 will be fulfilled.)

A verification of the last condition is a difficult problem. Therefore, in the present paper of question about construction of ring of invariants for arbitrary systems was not be considered. (The exception is made by the systems of types $(2 p, 2)$ and $(2 p+1,2)$, for which the rings of invariants were indicated in Subsections 7.2 and 7.3.)

Nevertheless, we can build the generators of quotient field $\mathbb{C}(A, B)^{\mathbb{S} L}$ for the systems $(A, B)$, which were considered in Section 5 . Knowledge of these generators already allows to solve the equivalence problem. (However, their number is not minimal; it is a main lack of conception of null-forms.)

\section{Description of invariants for system (2.1), (2.2)}

Below, we will use the ring of invariants of matrix pair $(C, A)$ with respect to action of group $\mathbb{S} L$. This ring can be got from algebra $\mathbb{C}[A, B]^{\mathbb{S} L}$ by replacements 
$B \rightarrow C^{T}$ and $A \rightarrow A^{T}$. We will designate this ring by the symbol $\mathbb{C}[C, A]^{\mathbb{S} L}$, where $\mathbb{S} L=\{S \times W\}=\mathbb{S} L(n, \mathbb{C}) \times \mathbb{S} L(p, \mathbb{C})$. In addition, for system $(2.1),(2.2)$ we will use the designation $(C, A, B)$. We will also call the system $(C, A, B)$ by a system of type $(p, n, m)$, where numbers $p, n$, and $m$ are dimensions of the output space, state space, and input space.

Note that results of this subsection can be got from the theorem about structure of generators of ring of invariants with respect to action (2.3) of group $\mathbb{S} L$ for one $(n \times n)$-matrix, $m$ column vectors and $p$ row vectors of dimension $n$ [37].

Introduce the matrices

$$
\begin{aligned}
& R_{1}(A, B)=\left(B, A B, \ldots, A^{n-1} B\right) \in \mathbb{C}^{n \times n m}, \\
& R_{2}(C, A)=\left(C^{T},(C A)^{T}, \ldots,\left(C A^{n-1}\right)^{T}\right)^{T} \in \mathbb{C}^{p n \times n}, \\
& R_{3}(C, A, B)=\left(C B, C A B, \ldots, C A^{n-1} B\right) \in \mathbb{C}^{p \times m p}, \\
& R_{4}(C, A, B)=\left((C B)^{T},(C A B)^{T}, \ldots,\left(C A^{n-1}\right)^{T} B^{T}\right)^{T} \in \mathbb{C}^{m p \times m} .
\end{aligned}
$$

Then action (2.3) of group $\mathbb{S} L$ on the space $\mathbb{C}^{n \times n} \times \mathbb{C}^{n \times m} \times \mathbb{C}^{p \times n}$ induce actions of the same group on spaces $\mathbb{C}^{n \times n m}, \mathbb{C}^{p n \times n}, \mathbb{C}^{p \times m p}$, and $\mathbb{C}^{m p \times m}$, which are given by the following formulas:

$$
\begin{aligned}
& \text { 1) } R_{1}\left(S^{-1} A S, S^{-1} B T\right)=S^{-1} \cdot R_{1}(A, B) \cdot\left(\begin{array}{lll}
T & & 0 \\
& \ddots & \\
0 & & T
\end{array}\right), \\
& \text { 2) } R_{2}\left(S^{-1} A S, W C S\right)=\left(\begin{array}{ccc}
W & & 0 \\
& \ddots & \\
0 & & W
\end{array}\right) \cdot R_{2}(C, A) \cdot S, \\
& \text { 3) } R_{3}\left(W C S, S^{-1} A S, S^{-1} B T\right)=W \cdot R_{3}(C, A, B) \cdot\left(\begin{array}{ccc}
T & & 0 \\
& \ddots & \\
0 & & T
\end{array}\right), \\
& \text { 4) } R_{4}\left(W C S, S^{-1} A S, S^{-1} B T\right)=\left(\begin{array}{ccc}
W & & 0 \\
& \ddots & \\
0 & & W
\end{array}\right) \cdot R_{4}(C, A, B) \cdot T .
\end{aligned}
$$

Further, as well as in Section 5, it is necessary to find decompositions of the representations

$$
\bigwedge^{n}\left(\bigoplus_{i=1}^{n} T\right), \bigwedge^{n}\left(\bigoplus_{i=1}^{n} W\right), \bigwedge^{p}\left(\bigoplus_{i=1}^{n} T\right), \bigwedge^{m}\left(\bigoplus_{i=1}^{n} W\right)
$$


on irreducible components. These decompositions are given by the following formulas:

$$
\begin{aligned}
& \bigwedge^{n}\left(\bigoplus_{i=1}^{n} T\right)=\underset{\omega_{1}=\left(n_{11}, \ldots, n_{1 d}\right)}{\bigoplus} r_{\omega_{1}} \wedge^{n_{11}} T \otimes \wedge^{n_{12}} T \otimes \ldots \otimes \wedge^{n_{1 d}} T, \\
& \bigwedge^{n}\left(\bigoplus_{i=1}^{n} W\right)=\underset{\omega_{2}=\left(n_{21}, \ldots, n_{2 d}\right)}{\bigoplus} r_{\omega_{2}} \wedge^{n_{21}} W \otimes \wedge^{n_{22}} W \otimes \ldots \otimes \wedge^{n_{2 d}} W, \\
& \bigwedge^{p}\left(\bigoplus_{i=1}^{n} T\right)=\underset{\omega_{1}=\left(n_{31}, \ldots, n_{3 d}\right)}{\bigoplus} r_{\omega_{3}} \wedge^{n_{31}} T \otimes \wedge^{n_{32}} T \otimes \ldots \otimes \wedge^{n_{3 d}} T, \\
& \bigwedge^{m}\left(\bigoplus_{i=1}^{n} W\right)=\underset{\omega_{4}=\left(n_{41}, \ldots, n_{4 d}\right)}{\bigoplus} r_{\omega_{4}} \wedge^{n_{41}} W \otimes \wedge^{n_{42}} W \otimes \ldots \otimes \wedge^{n_{4 d}} W .
\end{aligned}
$$

(Here a meaning of denotations the same that in Section 5.)

In future we will be restricted only to the case $m=p$.

Introduce the following invariants of group $\mathbb{S} L$ :

$$
K_{j}=\operatorname{det}\left(C A^{r-1} B\right), j=1, \ldots, r=\operatorname{Bin}(n, m) .
$$

Theorem 8.1. Let $(C, A, B)$ be a system of type $(n, m, p)$, where $m=p \leq n$. Then the ring of invariants $\mathbb{C}[C, A, B]^{\mathbb{S} L}$ of this system is generated:

- by the coefficients $a_{n}, \ldots, a_{n}$ of characteristic polynomial of matrix $A$;

- by the generators of ring $\mathbb{C}[A, B]^{\mathbb{S} L}$ essentially depending on $B$;

- by the generators of ring $\mathbb{C}[C, A]^{\mathbb{S L}}$ essentially depending on $C$;

- by invariants $K_{j}=\operatorname{det}\left(C A^{r-1} B\right), j=1, \ldots, r=\operatorname{Bin}(n, m)$.

Proof. We assume that we know rings of invariants $\mathbb{C}[A, B]^{\mathbb{S} L}$ of system $(A, B)$ and $\mathbb{C}[C, A]^{\mathbb{S} L}$ of systems $(C, A)$. Assume also that the matrix $A$ resulted to the diagonal form: $A=\operatorname{diag}\left(() \alpha_{1}, \ldots, \alpha_{n}\right)$. Then invariants (8.1) can be rewritten in the form:

$$
K_{j}=\sum_{1 \leq i_{1}<\ldots<i_{m} \leq n}\left(\alpha_{i_{1}}^{j} \cdot \ldots \cdot \alpha_{i_{n}}^{j}\right) B_{j} C_{j},
$$

where $B_{j}\left(C_{j}\right)$ are coordinates of polyvector $\wedge^{m} B$ (of polyvector $\left.\wedge^{m} C\right) ; j=1, \ldots, r$.

Rewrite equations (8.2) in the matrix form:

$$
\mathbf{K}=H\left(\alpha_{1}, \ldots, \alpha_{n}\right) \mathbf{D},
$$

where $H \in \mathbb{C}^{r \times r}, \mathbf{K}=\left(K_{1}, \ldots, K_{r}\right)^{T}, \mathbf{D}=\left(B_{1} C_{1}, \ldots, B_{r} C_{r}\right)^{T}$.

If $\operatorname{disc}\left(H\left(\alpha_{1}, \ldots, \alpha_{n}\right)\right)=0$, then we will replace the matrix $A$ by the matrix $A+\lambda E$, where $E$ is the identity matrix. Then by the choice $\lambda$ it is possible to obtain $\operatorname{disc}\left(H\left(\alpha_{1}+\lambda, \ldots, \alpha_{n}+\lambda\right)\right) \neq 0$ (see Theorem 6.2).

Thus, we can consider that $\operatorname{disc}\left(H\left(\alpha_{1}, \ldots, \alpha_{n}\right)\right) \neq 0$. In this case the vector $\mathbf{D}$ is uniquely determined from equations (8.3). Since the function $\operatorname{disc}\left(H\left(\alpha_{1}, \ldots, \alpha_{n}\right)\right)$ 
is regular, then from solvability of system (8.2) we get products of coordinates $B_{j}$ and $C_{j} ; j=1, \ldots, r$.

We can consider that the solutions of system (8.3) are seeking on some open set $\mathcal{L} \subset \mathbb{C}^{r}$ such that if $\wedge^{m} B \in \mathcal{L}$ and $\wedge^{m} C^{T} \in \mathcal{L}$, then $B_{j} C_{j}=W_{j} \neq 0$; $j=1, \ldots, r$. From here it follows that if coordinates of vector $\wedge^{m} B$ (or $\wedge^{m} C^{T}$ ) are known, then coordinates of vector $\wedge^{m} C^{T}$ (or $\wedge^{m} B$ ) are uniquely determined from the system of equations $B_{j} C_{j}=W_{j} \neq 0 ; j=1, \ldots, r$.

Further, in the same way as in the proof of Theorem 7.2, we use the Hilbert theorem [35] and the method of construction of invariants (8.1). The proof is finished.

It is possible to specify the results of Theorem 8.1 if to take advantage of Theorems 7.2 and 7.3. Two next theorems are the obvious corollaries of Theorem 8.1 .

Denote by $\mathbb{O}^{\mathbb{G}}(C, A, B) \subset \mathbb{S}$ an orbit of system $(C, A, B)$ with respect to action (2.3) of group $\mathbb{S} L$.

Note that the dimension of space of orbits $\mathbb{O}^{\mathbb{G}}(\mathbb{S})$ for system of type $(p, n, m)$ is given by the formula:

$$
\begin{gathered}
\operatorname{dim}_{\mathbb{C}} \mathbb{O}^{\mathbb{S} L}(\mathbb{S})=n(n+m+p)-\operatorname{dim}_{\mathbb{C}} \mathbb{S} L(n, \mathbb{C})-\operatorname{dim}_{\mathbb{C}} \mathbb{S} L(m, \mathbb{C})-\operatorname{dim}_{\mathbb{C}} \mathbb{S} L(p, \mathbb{C}) \\
=n(p+m)+3-m^{2}-p^{2} .
\end{gathered}
$$

Let $(C, A, B)$ be a system of type $(2,2 p, 2)$. In this case $\operatorname{dim}_{\mathbb{C}} \mathbb{O}^{\mathbb{S} L}(\mathbb{S})=8 p-5$.

Theorem 8.2. Let $(C, A, B)$ be a system of type $(2,2 p, 2)$, where $p \geq 1$. Then the ring of invariants $\mathbb{C}[C, A, B]^{\mathbb{S} L}$ of this system is generated:

- by $2 p$ coefficients $a_{1}, \ldots, a_{2 p}$ of characteristic polynomial of matrix $A$;

- by $2 p-2$ polynomials $\operatorname{det}\left(B, A B, \ldots, A^{p-1} B\right), \ldots, \operatorname{det}\left(B, A B, \ldots, A^{2 p-2} B\right)$;

- by $2 p-2$ polynomials $\operatorname{det}\left(C^{T},(C A)^{T}, \ldots,\left(C A^{p-1}\right)^{T}\right), \ldots, \operatorname{det}\left(C^{T},(C A)^{T}, \ldots\right.$, $\left.\left(C A^{2 p-2}\right)^{T}\right)$;

- by $2 p-1$ polynomials $\operatorname{det}(C B), \operatorname{det}(C A B), \ldots, \operatorname{det}\left(C A^{2 p-2} B\right)$.

The number $8 p-5$ of generators of ring $\mathbb{C}[C, A, B]^{\mathbb{S} L}$ is minimal.

Let $(C, A, B)$ be a system of type $(2,2 p+1,2)$. In this case $\operatorname{dim}_{\mathbb{C}} \mathbb{O}^{\mathbb{S} L}(\mathbb{S})=$ $8 p-1$.

Theorem 8.3. Let $(C, A, B)$ be a system of type $(2,2 p+1,2)$, where $p \geq 1$. Then the ring of invariants $\mathbb{C}[C, A, B]^{\mathbb{S} L}$ of this system is generated:

- by $2 p+1$ coefficients $a_{1}, \ldots, a_{2 p+1}$ of characteristic polynomial of matrix A;

- by $2 p-1$ polynomials

$$
\operatorname{det}\left(\begin{array}{cc}
\operatorname{det}\left(B, A B, \ldots, A^{p-1} B, A^{i} b_{1}\right) & \operatorname{det}\left(B, A B, \ldots, A^{p-1} B, A^{i} b_{2}\right) \\
\operatorname{det}\left(B, A B, \ldots, A^{p-1} B, A^{j} b_{1}\right) & \operatorname{det}\left(B, A B, \ldots, A^{p-1} B, A^{j} b_{2}\right)
\end{array}\right),
$$

where $i=p, p+1 \leq j \leq 2 p$ and $i=p+1, p+2 \leq j \leq 2 p$; 
- by $2 p-1$ polynomials

$$
\operatorname{det}\left(\begin{array}{cc}
\operatorname{det}\left(C^{T}, \ldots,\left(C A^{p-1}\right)^{T},\left(c_{1} A^{i}\right)^{T}\right)^{T} & \operatorname{det}\left(C^{T}, \ldots,\left(C A^{p-1}\right)^{T},\left(c_{2} A^{i}\right)^{T}\right)^{T} \\
\operatorname{det}\left(C^{T}, \ldots,\left(C A^{p-1}\right)^{T},\left(c_{1} A^{j}\right)^{T}\right)^{T} & \operatorname{det}\left(C^{T}, \ldots,\left(C A^{p-1}\right)^{T},\left(c_{2} A^{j}\right)^{T}\right)^{T}
\end{array}\right),
$$

where $i=p, p+1 \leq j \leq 2 p$ and $i=p+1, p+2 \leq j \leq 2 p$;

- by $2 p$ polynomials $\operatorname{det}(C B), \operatorname{det}(C A B), \ldots, \operatorname{det}\left(C A^{2 p-1} B\right)$.

The number $8 p-1$ of generators of ring $\mathbb{C}[C, A, B]^{\mathbb{S} L}$ is minimal.

Let $(C, A, B)$ be a system of type $(m, m+1, m)$ and let $a_{1}, \ldots, a_{m+1}$ be coefficients of characteristic polynomial of matrix $A$. In this case $\operatorname{dim}_{\mathbb{C}} \mathbb{O}^{\mathbb{S} L}(\mathbb{S})=2 m+3$.

Theorem 8.4. Let $(C, A, B)$ be a system of type $(m, m+1, m)$, where $m \geq 1$. Let also $R(A, B)$ be matrix (5.3). Then we have

$$
\begin{gathered}
\mathbb{C}[C, A, B]^{\mathbb{S} L} \\
=\mathbb{C}\left[a_{1}, \ldots, a_{m+1}, \operatorname{det} R(A, B), \operatorname{det} R\left(A^{T}, C^{T}\right), \operatorname{det}(C B), \ldots, \operatorname{det}\left(C A^{(m-1)} B\right],\right.
\end{gathered}
$$

where the number $2 m+3$ of generators is minimal. In addition, the set of all $\mathbb{S} L$-stable systems is given by the condition $\operatorname{det}\left(R(A, B) \cdot R\left(A^{T}, C^{T}\right)\right) \neq 0$.

Proof of the last assertion follows from Subsection 6.2. Here we have

$$
\begin{gathered}
\operatorname{det}\left(R(A, B) \cdot R\left(A^{T}, C^{T}\right)\right)=I(A, B) \cdot I\left(A^{T}, C^{T}\right) \\
=\operatorname{disc}(A) \underbrace{\Delta_{1 \ldots m}(B) \cdot \ldots \cdot \Delta_{2 \ldots m+1}(B)}_{m+1} \underbrace{\Delta_{1 \ldots m}(C) \cdot \ldots \cdot \Delta_{2 \ldots m+1}(C)}_{m+1} \neq 0 .
\end{gathered}
$$

\section{Equivalence of linear control systems}

The most invariants, which considered in the present article, are described in Theorem 6.1. It means that these invariants are minors of appropriate matrix $G(A, B)$ (or $R_{i}(A, B)$ ). From here it follows that all syzygies, which exist between invariants, always are automatically satisfied. It facilitates the search of minimal base of invariants (see Theorems $8.2-8.4$ ).

Although the problem of description of generators of ring of invariants in general case is not solved, nevertheless the equivalence problem, which is important for applications, got the solution. (It is here enough to know the generators of quotient field only.)

All said before we can sum up by the following obvious theorem.

Let $(C, A, B)$ be a system of type $(p, n, m)$. Let also the numbers $a_{1}(A), \ldots$, $a_{n}(A)$ be the coefficients of characteristic polynomial of matrix $A$. Denote by $I_{j}(A, B), j=1, \ldots, r_{B}$, the generators of quotient field $\mathbb{C}(A, B)^{\mathbb{S} L}$ depending on $B$ and denote by $P_{q}(C, A), q=1, \ldots, r_{C}$, the generators of quotient field $\mathbb{C}(C, A)^{\mathbb{S} L}$ depending on $C$. If $p=m$, then we denote by $K_{l}(C, A, B)=\operatorname{det}\left(C A^{l-1} B\right), l=$ $1, \ldots, r_{B C}$ the invariants depending on $B$ and $C$. 
Theorem 9.1. Let the positive integers $(n, m)$, where $n>m$, be coprime. Let also $p=m$. Then two systems $\left(C_{1}, A_{1}, B_{1}\right) \in \mathbb{S}_{\text {open }}$ and $\left(C_{2}, A_{2}, B_{2}\right) \in \mathbb{S}_{\text {open }}$ of type $(p, n, m)$ are equivalent if and only if there exist nonzero numbers $v$ and $t$ such that

$$
\begin{aligned}
& a_{1}\left(A_{1}\right)=a_{1}\left(A_{2}\right), \ldots, a_{n}\left(A_{1}\right)=a_{n}\left(A_{2}\right), \\
& I_{j}\left(A_{1}, B_{1}\right)=v I_{j}\left(A_{2}, B_{2}\right), j=1, \ldots, r_{B}=r_{C}, \\
& P_{q}\left(C_{1}, A_{1}\right)=t P_{q}\left(C_{2}, A_{2}\right), q=1, \ldots, r_{C}=r_{B}, \\
& K_{l}\left(C_{1}, A_{1}, B_{1}\right)=(v t)^{1 / r} K_{l}\left(C_{2}, A_{2}, B_{2}\right), l=1, \ldots, r_{B C},
\end{aligned}
$$

where $r=n \cdot \operatorname{Bin}(m, s), 1<s<m, s=n-d m$, $d$ is the integer part of $n / m$, and as the set $\mathbb{S}_{\text {open }}$ the set $\mathbb{F}_{s}$ of all $\mathbb{S} L$-stable systems can be taken .

Proof. We have that the numbers $n$ and $m$ are coprime and even one of invariants $I_{j}(A, B)\left(P_{q}(C, A)\right)$ is not equal to zero. Then from Theorems 6.1 and 6.3 it follows that there exists some subset of all $\mathbb{S} L$-stable systems of type $(n, m)((p, n))$, for which the equivalence problem has solution.

For some types of systems the generators of quotient field can be taken from Theorems 5.1, 5.4, and 5.5.

Another important problem, which was not solved, it is the search problem of set of all $\mathbb{S} L$-stable systems. (The solution was got only for systems $(A, B)$ of types $(4,2)$ and $(m+1, m)$.) However, it should be said that the set of all $\mathbb{S} L$-stable systems contains a subset, which is defined by the condition $\operatorname{disc}(A) \prod_{i=1}^{h_{B}}\left(\wedge^{m} B\right)_{i}$. $\prod_{j=1}^{h_{C}}\left(\wedge^{m} C\right)_{j} \neq 0$, where $\left(\wedge^{m} B\right)_{i}\left(\left(\wedge^{p} C\right)_{j}\right)$ are coordinates of polyvector $\wedge^{m} B$ (of polyvector $\left.\wedge^{p} C\right) ; h_{B}=\operatorname{Bin}(n, m), h_{C}=\operatorname{Bin}(n, p)$. In future the authors hope to solve the problem of description of all $\mathbb{S} L$-stable systems of type $(p, n, m), n>m$, $n>p$.

\section{Reconstruction of differential equations system on the known multivariate time series}

We assume that there are $n$ characteristics (measurements and computations) of some dynamic process : $z_{1}\left(t_{i}\right), \ldots, z_{n}\left(t_{i}\right), i=1,2, \ldots, N$. In addition, we also suppose that these measurements are noisy. Thus, we have multivariate time series

$$
z_{1}\left(t_{i}\right)=x_{1}\left(t_{i}\right)+\theta_{1}\left(t_{i}\right), \ldots, z_{n}\left(t_{i}\right)=x_{n}\left(t_{i}\right)+\theta_{n}\left(t_{i}\right),
$$

which defined for $\forall t_{i} \in\left(t_{1}, t_{N}\right)$. Here $\forall i=1,2, \ldots, N$, we have $t_{i}=i \Delta t$ and $\Delta t=\left(t_{N}-t_{1}\right) / N$. In addition, we suppose that $\theta_{1}\left(t_{i}\right), \ldots, \theta_{n}\left(t_{i}\right)$ are Gaussian (white) noises, unable by definition to produce statistically systematical errors [29] - [31], [34], [49].

Finally, we assume that $x_{1}\left(t_{i}\right), \ldots, x_{n}\left(t_{i}\right)$ is a discrete approximation of some curve $\mathbf{x}(t)=\left(x_{1}(t), \ldots, x_{n}(t)\right)^{T} \in \mathbb{R}^{n}$ [16], [29] - [31], [34], [49]. In the turn, 
it is assumed that the curve $\mathbf{x}(t)$ is a solution of some autonomous differential equations system. (The necessity of such description is dictated by the considerations resulted higher.)

Further, we use the procedure of determining unknown right-hand side of the system of differential equations (1.3), which was suggested in [16], [29] [31], [34], [36], [49]. This procedure is based on the least squares method and the fact that we know sufficient precision the components of $\mathbf{x}(t)$ and its derivative $\dot{\mathbf{x}}(t)$.

In view of the fact that number $N$ may be chosen arbitrary large, a high precision reconstruction may be achieved. Thus, we can expect that the solution of reconstructed system will be near the purified solution $\mathbf{x}(t)$.

However, it should be said that one important circumstance, which can arise up at a reconstruction, remained outside the attention of authors of article [34], [49]. The point is that in [34], [49] it is assumed that this interval $\left(t_{1}, t_{N}\right)$ is finite. If the problem of long-term prediction is considered, it is necessary to assume that $t_{N} \rightarrow \infty$. In this case a reconstruction must be fulfilled so that system (1.3) had the bounded solutions [2] - [6].

Now suppose that the dimension $n$ of phase space in which the dynamic process under study, is known. Assume also that $m$ different variables $(m<n)$ describing this process, for which time series can be measured, are also known. Then the matrices $A$ and $B$ of system (1.3) can be represented in the following form [5,20]:

$$
S^{-1} A S=\left(\begin{array}{ccc}
A_{11} & \cdots & A_{1 m} \\
\vdots & A_{i i} & \vdots \\
A_{m 1} & \cdots & A_{m m}
\end{array}\right), S^{-1} B T=\left(\begin{array}{ccc}
B_{11} & \cdots & 0 \\
\vdots & B_{i i} & \vdots \\
0 & \cdots & B_{m m}
\end{array}\right)
$$

where

$$
\begin{gathered}
A_{i i}=\left(\begin{array}{cccc}
0 & 1 & \cdots & 0 \\
\vdots & \vdots & \ddots & \vdots \\
0 & 0 & \cdots & 1 \\
-a_{i, \eta_{i-1}+1} & -a_{i, \eta_{i-1}+2} & \cdots & -a_{i, \eta_{i}}
\end{array}\right) \in \mathbb{R}^{\nu_{i} \times \nu_{i}} ; B_{i i}=\left(\begin{array}{c}
0 \\
\vdots \\
0 \\
1
\end{array}\right) \in \mathbb{R}^{\nu_{i}} \\
\eta_{0}=0, \eta_{1}=\nu_{1}, \eta_{2}=\nu_{1}+\nu_{2}, \ldots, \eta_{i}=\nu_{1}+\ldots+\nu_{i} \\
A_{i j}=\left(\begin{array}{ccc}
0 & \cdots & 0 \\
\vdots & \ddots & \vdots \\
0 & \cdots & 0 \\
-a_{i, \delta_{j-1}+1} & \cdots & -a_{i, \delta_{j}}
\end{array}\right) \in \mathbb{R}^{\nu_{i} \times \nu_{j}}, i \neq j \\
\delta_{0}=0, \delta_{1}=\nu_{1}, \delta_{2}=\nu_{1}+\nu_{2} \ldots, \delta_{i}=\nu_{1}+\ldots+\nu_{j} .
\end{gathered}
$$

Here integer positive numbers $\nu_{i}$ accept two values: $\nu_{i}=k+1$, if $i=1, \ldots, n-k m$ and $\nu_{i}=k$, if $i=n-k m+1, \ldots, m$, where $k$ is the integer part of number $n / m$; $i, j=1, \ldots, m$. 
All theory described higher allows to form the following procedure of reconstruction of differential equations system on the known multivariate time series (1.2) of process $\mathbf{P}(t)$ :

1. Let $m$ be the number of measured variables. By the embedding technique $([34,49])$ define the embedding dimension space $n>m$.

2. By a procedure of approximation of higher derivatives, compute a different composition from $n-m$ derivatives (these are missing or hidden variables) of the complementary $m$ known variables to the basis of the phase space (for example, $\left.x(t)=x_{1}, \dot{x}(t)=x_{2}, \ddot{x}(t)=x_{3}, \ldots\right)([16],[36])$.

3. Select the activation functions for system (1.3). After this, by the least square method ( [7], [16], [36]) compute the indeterminate coefficients of matrices $A, B$, and $C$ of system (1.3) reconsructed from the known multivariate time series (1.2).

4. Compose the linear control system (1.9) and by methods of Sections $5-9$ compute the polynomial invariants $a_{1}, \ldots, a_{n} ; I_{1}(A, B) \neq 0, \ldots, I_{q}(A, B) ; P_{1}(C, A) \neq$ $0, \ldots, P_{r}(C, A) ; K_{1}(C, A, B) \neq 0, \ldots, K_{s}(C, A, B)$ of this system.

5 . Repeat the steps $1-4$ of algorithm and compute new polynomial invariants

$$
\begin{gathered}
a_{1}^{\prime}, \ldots, a_{n}^{\prime} ; I_{1}^{\prime}\left(A^{\prime}, B^{\prime}\right) \neq 0, \operatorname{dots}, I_{q}^{\prime}\left(A^{\prime}, B^{\prime}\right) ; P_{1}^{\prime}\left(C^{\prime}, A^{\prime}\right) \neq 0, \ldots, \\
P_{r}^{\prime}\left(C^{\prime}, A^{\prime}\right) ; K_{1}^{\prime}\left(C^{\prime}, A^{\prime}, B^{\prime}\right) \neq 0, \ldots, K_{s}^{\prime}\left(C^{\prime}, A^{\prime}, B^{\prime}\right)
\end{gathered}
$$

of system (1.5) reconsructed from the known multivariate time series (1.4).

6. Verify the restrictions

$$
\left|a_{i}-a_{i}^{\prime}\right|<\epsilon,\left|I_{k} / I_{1}-I_{k}^{\prime} / I_{1}^{\prime}\right|<\epsilon,\left|P_{j} / P_{1}-P_{j}^{\prime} / P_{1}^{\prime}\right|<\epsilon,\left|K_{l} / K_{1}-K_{l}^{\prime} / K_{1}^{\prime}\right|<\epsilon,
$$

where $i=1, \ldots, n ; k=2, \ldots, q ; j=2, \ldots, r ; l=2, \ldots, s$. (It is here taken into account that among invariants $I_{k}$ there is even one nonzero. We assumed that it is the invariant $I_{1}$. The same assumption must be satisfied for invariants $P_{j}$ and $K_{l}$.) If they are faithful, then the problem of adequacy of neural network models (1.3) and (1.5) can considered solved.

\subsection{Reconstruction of dynamic processes in a contact electric network}

In article [43] a question about construction of regulator for stabilization of voltage in a contact network was studied. In this article the system of differential equations for the design of behavior of current (I) and voltage (U) in the mentioned netwowk was constructed. However, the problem of adequacy of the got model and real dynamics of electric processes in the contact network was not investigated.

Below, we will remove the indicated lack. For this purpose we apply the method of invariant reconstruction of differential equations to the process shown on the following Fig.2(a1).

In most practical cases of the reconstruction of differential equations according to the results of measurements of certain variables, it is impossible to change the 
composition of the measuring instruments. This means that the representation of the constructed model in other variables is also impossible. Therefore, the question of checking the adequacy of the model and the real system remains open.

In this paper, we propose the following method for checking the adequacy of the model and the real system.

The dynamics of arbitrary autonomous system of differential equations is fully determined by the parameters of this system.

Let $\mathbb{K}$ be a set of restrictions on the parameters of system (1.3), which determines its desired behavior. In the general case the restrictions are included in $\mathbb{K}$ should be the functions of invariants of system (1.3). (For example, the behavior of any linear dynamic system can be described using the coefficients of the characteristic polynomial, which, in turn, are invariants with respect to changes of variables of this system.)

At present, the construction of basis of polynomial invariants of an arbitrary polynomial system of differential equations is an unsolved problem. Therefore, we can use only a part of the polynomial invariants that are constructed in this article. In this regard, we propose the following methodology for checking the adequacy of the model and the real system.

1. Using the method of least squares to restore some system $\left(\mathcal{S}_{1}\right)$ of differential equations according to the measurement results.

2. Repeat measurements of the same dynamic characteristics of the process under study. After that, perform a reconstruction of new system differential equations $\left(\mathcal{S}_{2}\right)$.

3. For systems $\left(\mathcal{S}_{1}\right),\left(\mathcal{S}_{2}\right)$ check the implementation of inequalities of set $\mathbb{K}$.

4. If the current-voltage characteristics of systems $\left(\mathcal{S}_{1}\right)$ and $\left(\mathcal{S}_{2}\right)$ are equivalent, then it can be argued that the system reconstructed from measurement results adequately describes the dynamics of the real process in the contact network.

We will assume that we can measure the voltage and current, and also if it is possible other dynamic characteristics of contact electric network. We also suppose that among these characteristics can be derivatives with respect to $t$ from the voltage and current. (If the derivatives can not be measured, it is assumed that there exist smooth enough approximations of these derivatives.)

In [43] a structure of the process of represented on Fig. 2 (a2) was described by the following system of differential equations:

$$
\left\{\begin{array}{l}
\dot{x}_{1}(t)=x_{2} \\
\dot{x}_{2}(t)=a_{20}+a_{21} x_{1}+a_{22} x_{2}+a_{23} x_{3}+a_{24} x_{4}+b_{11} x_{1}^{2}+b_{12} x_{1} x_{2} \\
\dot{x}_{3}(t)=x_{4} \\
\dot{x}_{4}(t)=a_{40}+a_{41} x_{1}+a_{42} x_{2}+a_{43} x_{3}+a_{44} x_{4}+b_{22} x_{1}^{2}+b_{21} x_{1} x_{2} .
\end{array}\right.
$$




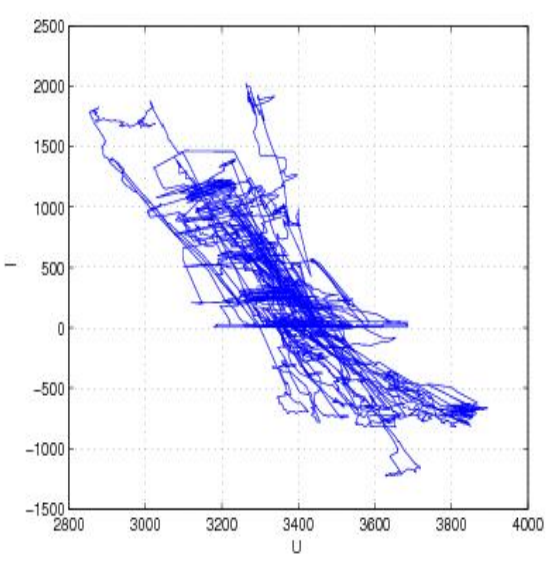

(a1)

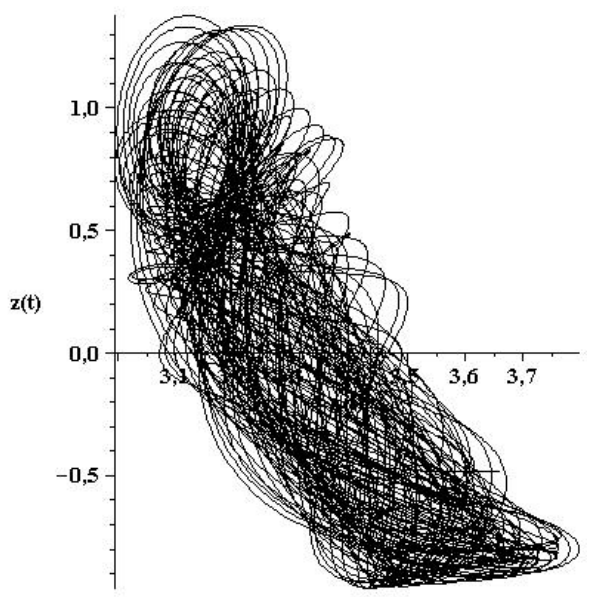

(a2)

Fig. 10.2. A current-voltage characteristic ( $U-I$ characteristic) of contact network: (a1) experimental data; (a2) modeling after14000 measurements for system (10.5) in Kv and Ka [7,43]; here $x=U, z=I$.

The system got as a result of reconstruction has the following form:

$$
\left\{\begin{aligned}
\dot{x}(t)= & y(t) \\
\dot{y}(t)= & 0.0193-0.0072 x(t)+0.0218 y(t)-0.000814 z(t)+0.0057 u(t) \\
& -0.0039 x(t) y(t)+0.000422 x^{2}(t) \\
\dot{z}(t)= & u(t) \\
\dot{u}(t)= & 0.0294-0.0145 x(t)-0.8506 y(t)-0.0019 z(t)-0.0095 u(t) \\
& +0.2380 x(t) y(t)+0.0017 x^{2}(t) .
\end{aligned}\right.
$$

For verification of conditions Theorem 9.1 we will carry beginning of coordinates of system $(10.5)$ in the equilibrium point $(3.335950,0,-0.027746,0)$. In the total we obtain such system:

$$
\left\{\begin{aligned}
\dot{x}(t)= & y(t) \\
\dot{y}(t)= & -0.004384 x(t)+0.008790 y(t)-0.000814 z(t)+0.005700 u(t) \\
& -0.00097(x(t)+y(t))^{2}+0.00097(x(t)-y(t))^{2}+0.000422 x^{2}(t), \\
\dot{z}(t)= & u(t) \\
\dot{u}(t)= & -0.003158 x(t)-0.056644 y(t)-0.0019 z(t)-0.0095 u(t) \\
& +0.05950(x(t)+y(t))^{2}-0.05950(x(t)-y(t))^{2}+0.0017 x^{2}
\end{aligned}\right.
$$

Let the activation function for system $(10.6)$ be $u_{i}=(x \pm y)^{2} ; i=1,2 ; u_{3}=x^{2}$. In this case for system (1.9) the matrices $A, B$, and $C$ have the following forms:

$$
A=\left(\begin{array}{cccc}
0 & 1 & 0 & 0 \\
-0.004384 & 0.008790 & -0.000814 & 0.005700 \\
0 & 0 & 0 & 1 \\
-0.003158 & -0.056644 & -0.001900 & -0.009500
\end{array}\right) \text {, }
$$




$$
B=\left(\begin{array}{ccc}
0 & 0 & 0 \\
-0.00097 & 0.00097 & 0.000422 \\
0 & 0 & 0 \\
0.059500 & -0.059500 & 0.001700
\end{array}\right), C^{T}=\left(\begin{array}{ccc}
1 & 1 & 1 \\
0 & 1 & -1 \\
0 & 0 & 0 \\
0 & 0 & 0
\end{array}\right)
$$

Note that for system (10.7) we have $m=p=3$ and $\operatorname{rank} B=\operatorname{rank} C=2<$ 3 . Therefore, invariant analysis of such system cannot be performed only using Theorem 9.1. In this case, we restrict ourselves to a visual comparison of phase portraits (see Fig.2(a2)) of system (10.5) with real process presented on Fig.2(a1).

\subsection{Example}

In accordance with the results given in $[7,43]$, the process presented on Fig. 2(a1) can be described by the following system of differential equations:

$$
\left\{\begin{array}{l}
\dot{x}_{1}(t)=x_{2}, \\
\dot{x}_{2}(t)=a_{20}+a_{21} x_{1}+a_{22} x_{2}+a_{23} x_{3}+a_{24} x_{4}+b_{11} x_{2}^{2}+b_{12} x_{4}^{2}, \\
\dot{x}_{3}(t)=x_{4} \\
\dot{x}_{4}(t)=a_{40}+a_{41} x_{1}+a_{42} x_{2}+a_{43} x_{3}+a_{44} x_{4}+b_{22} x_{2}^{2}+b_{21} x_{4}^{2} .
\end{array}\right.
$$

Here $x_{1}=U, x_{2}=\dot{U}, x_{3}=I, x_{4}=\dot{I}$.

The reconstructed system obtained from the results of 14000 measurements can be presented in the following form:

$$
\left\{\begin{aligned}
\dot{x}(t)= & y(t) \\
\dot{y}(t)= & 0.00676-0.0018348 x(t)+0.005384 y(t)-0.00005 z(t)+0.001827 u(t) \\
& +0.40864 y^{2}(t)-0.02409 u^{2}(t) \\
\dot{z}(t)= & u(t), \\
\dot{u}(t)= & 0.028596-0.000646 x(t)-0.03005 y(t)-0.001789 z(t)-0.00628 u(t) \\
& -2.50578 y^{2}(t)+0.06470 u^{2}(t) .
\end{aligned}\right.
$$

Here $x=x_{1}=U, y=x_{2}=\dot{x}=\dot{U}, z=x_{3}=I, u=x_{4}=\dot{z}=\dot{I}$.

For verification of conditions Theorem 9.1 we will carry beginning of coordinates of system (10.9) in the equilibrium point $(3.67857,0,0.027012,0)$. In the total we obtain such system:

$$
\left\{\begin{aligned}
\dot{x}(t)= & y(t) \\
\dot{y}(t)= & -0.0018348 x(t)+0.005384 y(t)-0.00005 z(t)+0.001827 u(t) \\
& +0.40864 y^{2}(t)-0.02409 u^{2}(t) \\
\dot{z}(t)= & u(t) \\
\dot{u}(t)= & -0.000646 x(t)-0.03005 y(t)-0.001789 z(t)-0.00628 u(t) \\
& -2.50578 y^{2}(t)+0.06470 u^{2}(t)
\end{aligned}\right.
$$

Let the activation function for system $(10.10)$ be $u_{i}=z_{i}^{2} ; i=1, \ldots, 3$. In this 
case for system (1.9) the matrices $A, B$, and $C$ have the following forms:

$$
\begin{gathered}
A=\left(\begin{array}{cccc}
0 & 1 & 0 & 0 \\
-0.001835 & 0.005384 & -0.00005 & 0.001827 \\
0 & 0 & 0 & 1 \\
-0.000646 & -0.030050 & -0.001789 & -0.006280
\end{array}\right), \\
B=\left(\begin{array}{cc}
0 & 0 \\
0.408640 & -0.024090 \\
0 & 0 \\
-2.505780 & 0.064700
\end{array}\right), C^{T}=\left(\begin{array}{ll}
0 & 0 \\
1 & 0 \\
0 & 0 \\
0 & 1
\end{array}\right) .
\end{gathered}
$$

System (10.9) has the following invariants:

$$
\begin{gathered}
a_{1}=-0.000914, a_{2}=0.001367, a_{3}=0 ., a_{4}=0 . ; I_{1}=-0.001612, I_{2}=0 . ; \\
P_{1}=0.000003, P_{2}=0 . ; K_{1}=-0.034036, K_{2}=K_{3}=K_{4}=K_{5}=0 .
\end{gathered}
$$

Now we will consider the modeling of the dynamic process in Fig.2(a1), but using time series only the first 10000 measurements. In this case, we get the following system:

$$
\left\{\begin{aligned}
\dot{x}(t)= & y(t) \\
\dot{y}(t)= & 0.006-0.0017348 x(t)+0.005784 y(t)-0.00004 z(t)+0.001927 u(t) \\
& +0.30864 y^{2}(t)-0.02409 u^{2}(t) \\
\dot{z}(t)= & u(t) \\
\dot{u}(t)= & 0.028596-0.000546 x(t)-0.02005 y(t)-0.001789 z(t)-0.00528 u(t) \\
& -2.50578 y^{2}(t)+0.06370 u^{2}(t)
\end{aligned}\right.
$$

For this system the equilibrium point is $(3.44600,0,0.54672,0)$. Now repeating the procedure for system (10.9) described above in Subsection 10.2, we arrive at such matrices of system (1.9):

$$
\begin{gathered}
A=\left(\begin{array}{cccc}
0 & 1 & 0 & 0 \\
-0.001735 & 0.005738 & -0.00004 & 0.001927 \\
0 & 0 & 0 & 1 \\
-0.000546 & -0.020050 & -0.001789 & -0.006658
\end{array}\right), \\
B=\left(\begin{array}{cc}
0 & 0 \\
0.308640 & -0.024090 \\
0 & 0 \\
-2.505780 & 0.063700
\end{array}\right), C^{T}=\left(\begin{array}{ll}
0 & 0 \\
1 & 0 \\
0 & 0 \\
0 & 1
\end{array}\right) .
\end{gathered}
$$

System (10.11) has the following invariants:

$$
\begin{gathered}
a_{1}=-0.000921, a_{2}=0.003532, a_{3}=0 ., a_{4}=0 . ; I_{1}=-0.001657, I_{2}=0 . ; \\
P_{1}=0.000003, P_{2}=0 . ; K_{1}=-0.034016, K_{2}=K_{3}=K_{4}=K_{5}=0 .
\end{gathered}
$$




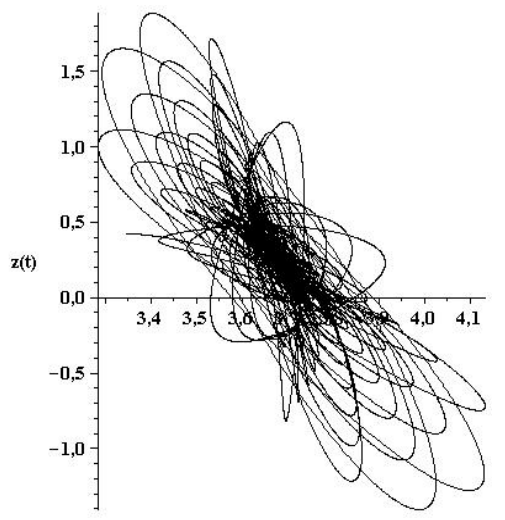

(a1)

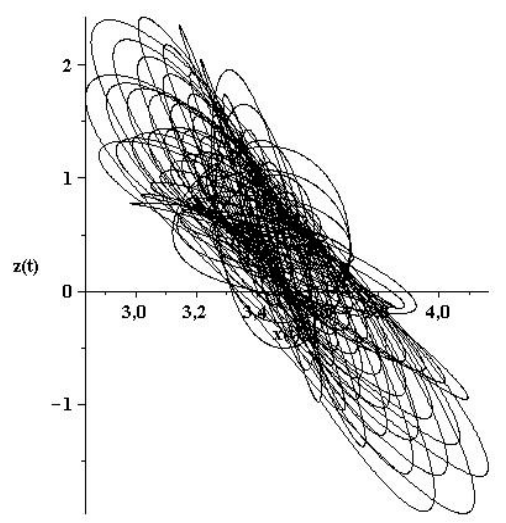

(a2)

Fig. 10.3. The current-voltage characteristics of contact electric network modeling experimental characteristic on Fig.2 at different lengths of time series: (a1) 14000 measurements for system (10.9); (a2) 10000 measurements for system (10.11)

The verification of the inequalities (10.3) shows that they are valid at $\epsilon=$ $0.1 \cdot 10^{-2}$. This circumstance means that for describing the process shown in Fig.2 can be used any from systems (10.9) or (10.11). The last statement can be confirmed by Fig.3 (here $z=I$ and $x=U$ ).

\section{Conclusion}

The results given above allow us to draw the following conclusions.

1. The problem of description of algebraic invariants for the linear control system is solved.

2. With the help of these invariants, the equivalence problem of two nonlinear systems obtained from results of studies of the corresponding time series is also solved.

Indeed, consider two nonlinear systems of 4 th order:

$$
\left\{\begin{array}{l}
\dot{x}_{1}(t)=x_{2} \\
\dot{x}_{2}(t)=a_{21} x_{1}+a_{22} x_{2}+a_{23} x_{3}+a_{24} x_{4}+v_{1}\left(x_{1}, \ldots, x_{4}\right), \\
\dot{x}_{3}(t)=x_{4} \\
\dot{x}_{4}(t)=a_{41} x_{1}+a_{42} x_{2}+a_{43} x_{3}+a_{44} x_{4}+v_{2}\left(x_{1}, \ldots, x_{4}\right)
\end{array}\right.
$$

and

$$
\left\{\begin{array}{l}
\dot{x}_{1}(t)=x_{2} \\
\dot{x}_{2}(t)=x_{3} \\
\dot{x}_{3}(t)=a_{31}^{\prime} x_{1}+a_{32}^{\prime} x_{2}+a_{33}^{\prime} x_{3}+a_{34}^{\prime} x_{4}+v_{1}^{\prime}\left(x_{1}, \ldots, x_{4}\right), \\
\dot{x}_{4}(t)=a_{41}^{\prime} x_{1}+a_{42}^{\prime} x_{2}+a_{43}^{\prime} x_{3}+a_{34}^{\prime} x_{4}+v_{2}^{\prime}\left(x_{1}, \ldots, x_{4}\right),
\end{array}\right.
$$

where $v_{1}(\ldots), v_{2}(\ldots)$ and $v_{1}^{\prime}(\ldots), v_{2}^{\prime}(\ldots)$ are nonlinear functions. 
Invariants of systems (11.1) and (11.2) in accordance with Theorem 7.2 look like: $a_{1}, \ldots, a_{4}, I_{1}=-1, I_{2}=-a_{22} a_{44}+a_{24} a_{42}$, and $a_{1}^{\prime}, \ldots, a_{4}^{\prime}, I_{1}^{\prime}=0, I_{2}^{\prime}=-a_{34}^{\prime}$.

Since $I_{1} \neq I_{1}^{\prime}$, then these systems are not equivalent for any nonlinearities within them. From here it follows that the structure of any system of differential equations obtained as a result of reconstruction is uniquely determined by the embedding dimension space $n$ and the number of independent measured variables $m(n>m)$ [7]. This structure is represented by matrices $A$ and $B$ (see (10.2)).

3. Note that using the invariants of system (1.9), it is impossible to fully describe system (1.3). (For this purpose, it is necessary to find the basis of all polynomial invariants of this system.) However, information about the behavior of measured variables already contains information about non-linearities that determine real process in the contact electric network. Therefore, the matrices $A, B$ and $C$ of system (1.9) determine the linear part and the structure of the nonlinear part of system (1.3).

4. As the considered examples show, the invariant reconstruction method is also suitable when we are dealing with chaotic processes taking place in the contact network.

\section{References}

1. M. BADER, Quivers, geometric invariant theory, and moduli of linear dynamical systems, Linear Algebra Appl., 428(2008), 2424 - 2454.

2. V. Ye. Belozyorov, Design of linear feedback for bilinear control systems, Int. J. Applied Mathematics and Computer Science, 12(4)(2002), 493 - 511.

3. V. Ye. Belozyorov, S. A. Volkova, Geometric approach to the problem of stabilization of control systems, Dnipro University Press, 2006 (in Russian).

4. V. Ye. Belozyonov, Invariant approach to an existence problem of nontrivial asymptotic stability cone, Canadien Applied Mathematics Quarterly, 15(2007), 125 $-168$.

5. V. Ye. Belozyorov, New solution method of linear static output feedback design problem for linear control systems, Linear Algebra Appl., 504(2016), 204 - 227.

6. V. Ye. Belozyorov, Invariant approach to the solvability of feedback design problem for linear control systems, Modelling, Dnipro National University Press, 25(2017), $58-69$.

7. V. Ye. Belozyorov, Ye. M. Kosariev, M. M. Pulin, V. G. Sychenko, V. G. ZAYTSEV. A new mathematical model of dynamic process in direct current traction power supply system, Journal of Optimization, Differential Equations and Their Applications (JODEA), 27(1) (2019), $21-55$.

8. A. E. Browder, J. Draisma, M. Popoviciu, The degrees of a system of parameters of the ring of invariants of a binary form, Tranform. Groups, 20(2015), $953-967$.

9. C. I. Byrnes, N. E. Hurt, On the moduli of linear dynamical systems, Studies in Analysis, Adv. Math. Suppl. Stud., Academic Press, New York, 4(1979), 83 - 122.

10. B. De BRUYN, On polyvectors of vector spaces and hyperplanes of projective Grassmannians, Linear Algebra Appl., 435(2011), 1055 - 1084.

11. R. T. Q. Chen, Y. Rubanova, J. Bettencourt, D. Duvenaud, Neural ordinary differential equations, arXiv preprint arXiv:1806.07366v5[cs.LG], (2019), $1-18$. 
12. D. F. Delchamps, Global structure of families of multivariable linear systems with an application to identification, Math. Systems Theory, 18(1985), 329 - 380.

13. S. FRIEDLAND, Classification of linear systems, Contemporary Mathematics, 47(1985), $131-147$.

14. P. A. Fuhrmann, U. Helmke, Equivalence conditions for behaviors and Kronecker canonical form, Math. Control Signals Syst, 22(2011), 267 - 293.

15. F. R. Gantmacher, The Theory of Matrices, AMS Chelsea Publising, Providence, Rhode Island, 2000.

16. N. V. Georgiev, P. N. Gospodinov, V. G. Petrov, Multi-variant time series based reconstruction of dynamical systems, Advanced Modeling and Optimization, 8 (2006), $53-64$.

17. S. HaYkin, Neural Networks. A Comprehensive Foundation, Second Edition, Pearson Education, Prentice Hall, 2005.

18. M. HAZEWINKEL, (Fine)moduli (spaces) for linear systems: what are they and what are they good for? Geometrical Methods for the Study of Linear Systems, (Proc. NATO Adv. Study Inst., Harvard Univ., Cambridge, Mass, 1979), NATO Adv. Study Inst. Ser., Ser. C: Math. Phys. Sci., Reidel, Dordrecht, 62(1980), 125 - 193.

19. M. HAZEWINKEL, C. MARTin, Representations of the symmetric group, the specialization order, systems and grassmann manifolds, L'Enseingnement Mathematique, 29(1983), 53 - 87 .

20. M. HAZEWINKEL, Lectures on invariants, representations and Lie algebras in systems and control theory, Lecture Notes in Mathematics, Springer-Verlag, Berlin, Heidelberg, New York, 1029, 1984.

21. U. HeLmKe, The topology of a moduli space for linear dynamical systems, Comment. Math. Helvetici, 60(1985), $630-655$.

22. U. HeLmKe, The topology of the moduli space for reachable linear dynamical systems: the complex case, Math. Systems Theory, 19(1986), 155 - 187.

23. U. Helmke, A compactification of the space of rational transfer functions by singular systems, J. Math. Syst. Estim.Control, 3(4)(1993), 459 - 472.

24. D. Hinrichsen, D. Pratzel-Wolters, State and input transformations for reachable systems: a polynomial approach, Contemporary Mathematics, 47(1985) , $217-239$.

25. J. J. Hopfield, D.W. TANK, Computing with neural circuits: a model, Science, New Series, 233(4764)(1985), 625 - 633.

26. E. M. IzHIKevich, Dynamical Systems in Neuroscience: The Geometry of Excitability and Bursting, The MIT Press Cambridge, Massachusetts, London, England, 2007.

27. J. JiA, A. R. Benson, Neural jump stochastic differential equations, arXiv preprint arXiv:1905.10403v3[cs.LG], (2020), 1 - 14.

28. R. E. KALMAN, Algebraic geometric description of the class of linear systems of constant dimension, Proceedings of the Annual Princeton Conference on Information Sciences and Systems, 8(1974), 189 - 192.

29. Y.- C.LAI, N. YE, Recent developments in chaotic time series analysis, Int. J. Bifurc. Chaos, 13 (2003), 1383 - 1422.

30. M. LI, Fractal time series - a tutorial review, Mathematical Problems in Engineering, 2010 (2010), 157264-1 - 26.

31. Z. LIU, Chaotic time series analysis, Mathematical Problems in Engineering, 2010 (2010), 720190-1 - 31. 
32. V. G. LOMADZE, Finite-dimensional time-invariant linear dynamical systems: algebraic theory, Acta Appl. Math., 19(2) (1990), 149 - 201.

33. W. Manthey, U. Helmke, Bruhat canonical form for linear systems, Linear Algebra Appl., 425(2007), $261-282$.

34. N. Marwan, M. Romano, M. Thiel, J. Kurths, Recurrence plots for the analysis of complex systems, Physics Reports, 438 (2007), 237 - 329.

35. D. Mumford, J. Fogarty, Geometric Invariant Theory, Second Enlarged Edition, Springer-Verlag, Berlin, Heidelberg, New York, 1982.

36. V. G. Petrov, J. Kurths, N. V. Georgiev, Reconstructing differential equations from a time series, Int. J. Bifurc. Chaos, 13 (2003), 3307 - 3323.

37. C. Processi, The invariant theory of $n \times n$-matrices, Advances in Math., 19(1976), $306-381$.

38. M. S. Ravi, J. Rosenthal, X. Wang, Dynamic pole assigment and Schubert calculus, SIAM J. Control and Opt., 34(3)(1996), 813 - 832.

39. M. S. Ravi, J. Rosenthal, U. Helmke, Output feedback invariants, Linear Algebra Appl., 351 - 352(2002), 623 - 637.

40. L. E. RENNER, Orbits and invariants of visible group actions, Tranform. Groups, $\mathbf{1 7}(2012), 1191-1208$.

41. J. Rosenthal, A compactification of the space of multivariable linear systems using geometric invariant theory, J. Math. Syst. Estim. Control, 2(1)(1992), 111 - 121.

42. J. Rosenthal, X. WAng, Output feedback pole placement with dynamic compensator, IEEE Trans. Autom. Control, AC-41(2)(1996), 830 - 843.

43. V. Sychenko, V. Kuznetsov, Ye. Kosariev, P. Hubskyi, V. Belozyorov, V. ZAYTSEV, M. PUlin, Development of an approach to ensure stability of the traction direct current system, Eastern-European Journal of Enterprise Technologies, 95(5)(2018), $47-57$.

44. T. A. Springer, Invariant Theory, Springer-Verlag, New York, 1977.

45. A. Tannenbaum, Invariance and System Theory: Algebraic and Geometric Aspects, Lecture Notes in Mathematics, Springer-Verlag, Berlin, Heidelberg, New York, 845(1981), $1-180$.

46. A. TAnnenbaum, Invariant theory and families of dynamical systems, Mathematical System Theory - The Influence of R. E. Kalman, Springer, New York, (1991), $327-345$.

47. A. TAnnenbaum, On the stabilizer subgroups of a pair of matrices, Linear Algebra Appl., 50(1983), 527 - 544 .

48. N.-E. TATAR, Hopfield neural networks with unbounded monotone activation functions, Hindawi Publishing Corporation. Advances in Artificial Neural Systems, 2012, 2012, 571358-1 - 5.

49. C. L. WebBer, N. MARWAn (EDS), Reccurence Quantification Analysis. Theory and Best Practice, Springer, NY, Dordrecht, Heidelberg, London, 2015.

50. W. M. Wonham, Linear Multivariable Control. A Geometric Approach, SpringerVerlag, New-York, 1985.

51. H. Zhang, X. Gao, J. Unterman, T. Arodz, Approximation capabilities of neural ordinary differential equations, arXiv preprint arXiv: 1907.12998v1[cs.LG], (2019), $1-11$. 